Aus der Abteilung Unfallchirurgie, Plastische und Wiederherstellungschirurgie

(Prof. Dr. med. K. M. Stürmer)

im Zentrum Chirurgie

der Medizinischen Fakultät der Universität Göttingen

\title{
Operative Therapie der ligamentären vorderen Kreuzbandruptur im Wachstumsalter - klinische Ergebnisse einer Multicenterstudie bei Patienten bis zu zwölf Jahren und systematische Analyse der Literatur
}

\author{
INAUGURAL-DISSERTATION \\ zur Erlangung des Doktorgrades \\ der Medizinischen Fakultät \\ der Georg-August-Universität zu Göttingen
}

vorgelegt von

Immanuel von Stietencron

aus

Filderstadt

Göttingen 2012 
Dekan:

1. Berichterstatter:

2. Berichterstatterin:
Prof. Dr. med. C. Frömmel

Prof. Dr. med. K.-H. Frosch

Prof. Dr. med. A.-K. Hell

Tag der mündlichen Prüfung: 14. August 2012 


\section{Inhaltsverzeichnis}

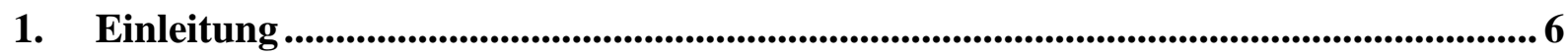

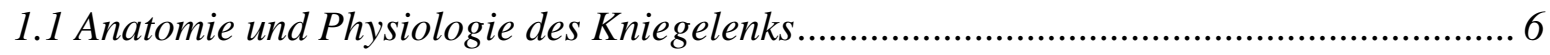

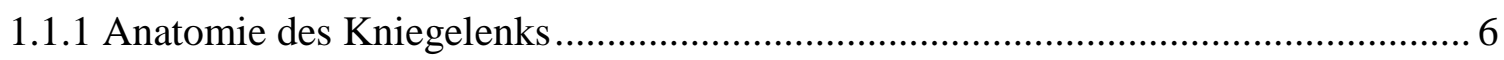

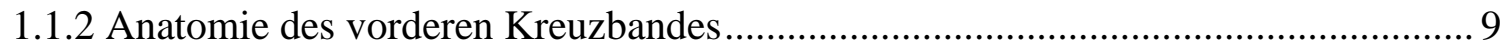

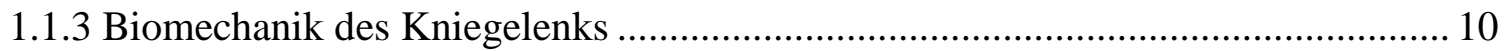

1.1.4 Funktion des vorderen Kreuzbandes ...................................................................... 11

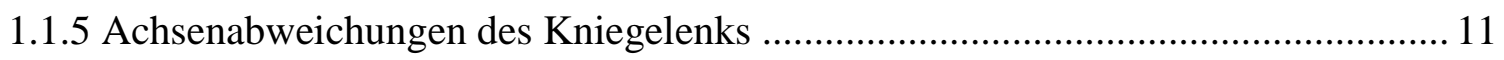

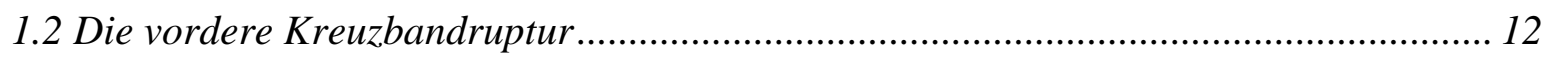

1.3 Verletzungsmuster der vorderen Kreuzbandruptur im Wachstumsalter....................... 13

1.4 Therapiemöglichkeiten der ligamentären vorderen Kreuzbandruptur im

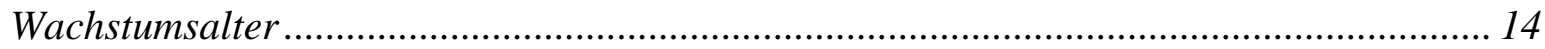

1.4.1 Besonderheiten, die sich für die Therapie bei Vorliegen offener Wachstumsfugen

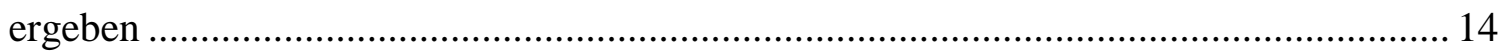

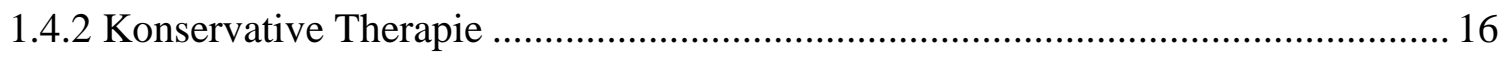

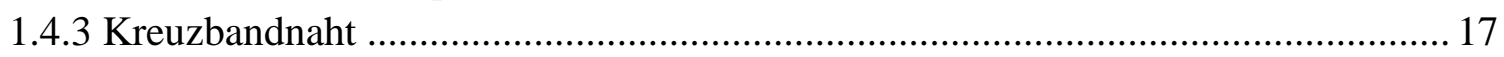

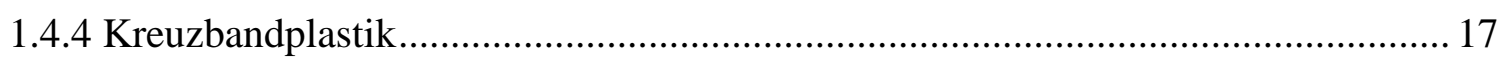

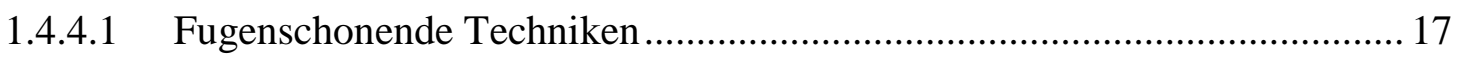

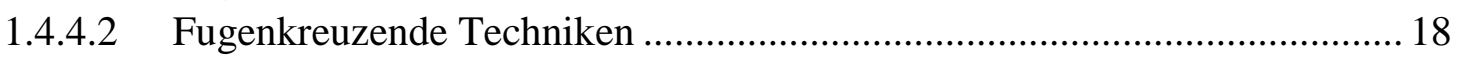

1.4.4.3 Partiell fugenkreuzende Techniken.......................................................... 18

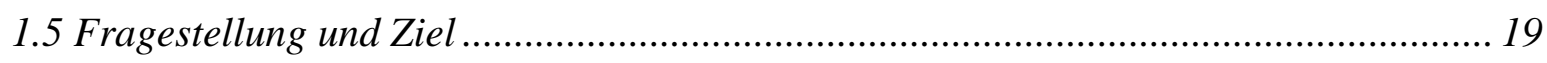

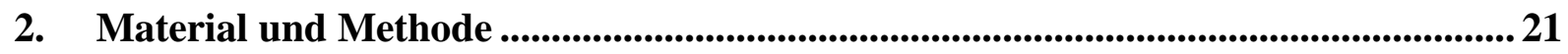

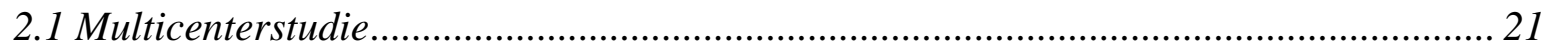

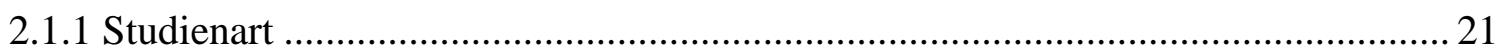

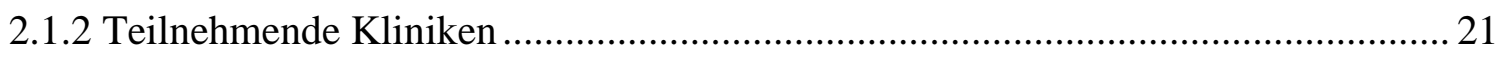

2.1.3 Informationsübermittlung über die Homepage www.kreuzband-kind.de............... 21

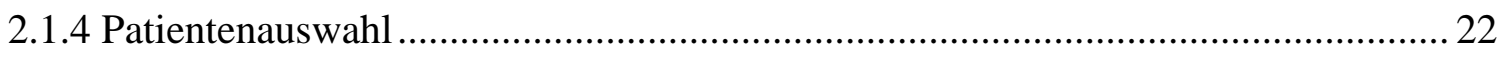

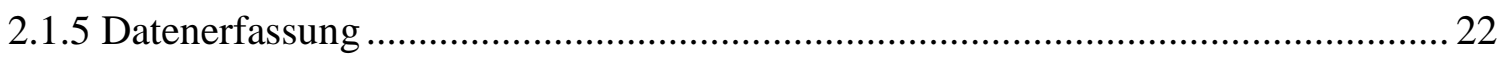

2.1.5.1 Instrumenteller Lachman-Test mit dem KT-1000-Arthrometer ................. 24

2.1.5.2 Lysholm-Score ......................................................................................... 24

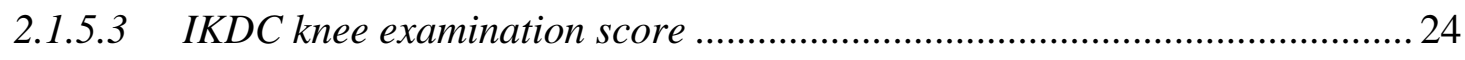

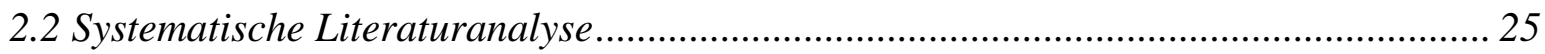

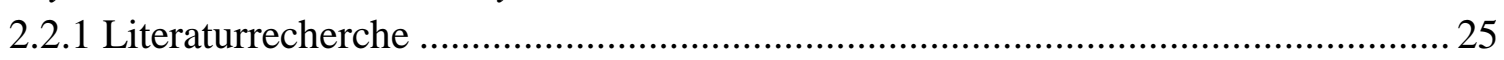

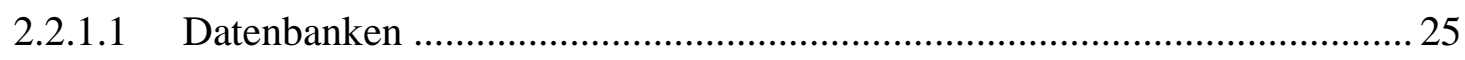

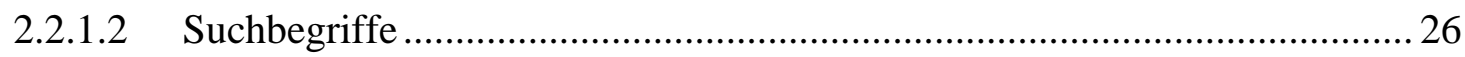

2.2.1.3 Auswahl der Publikationen .................................................................... 27

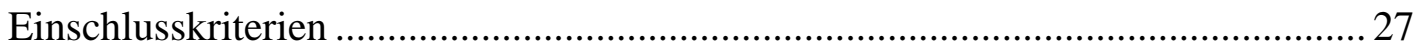

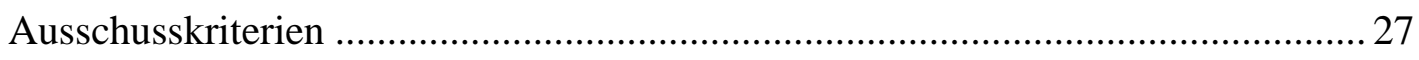

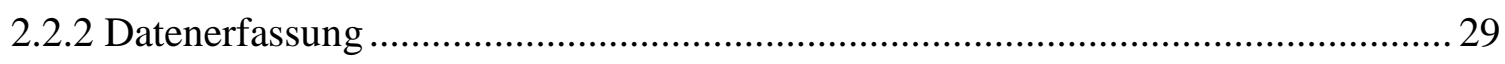


2.2.2.1 Berechnung der untersuchten Größen aus den einzelnen Artikeln. .33

3. Ergebnisse

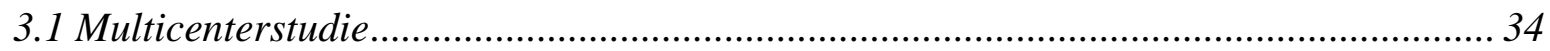

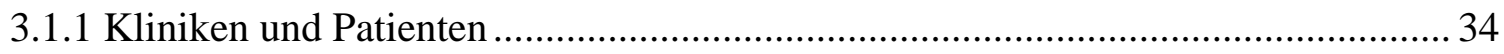

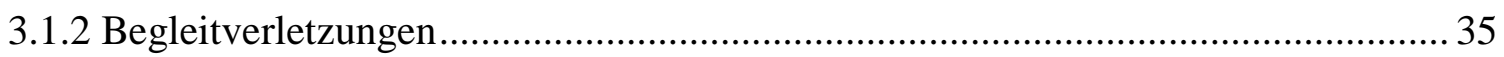

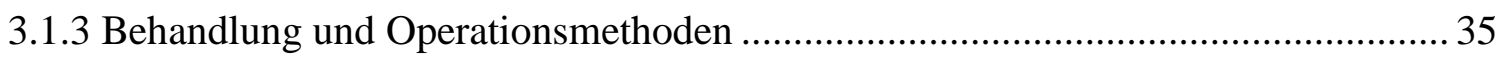

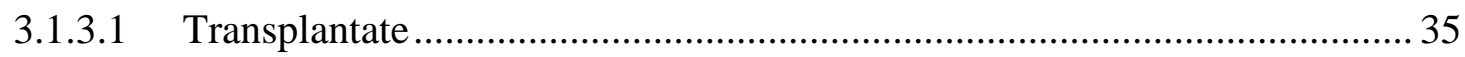

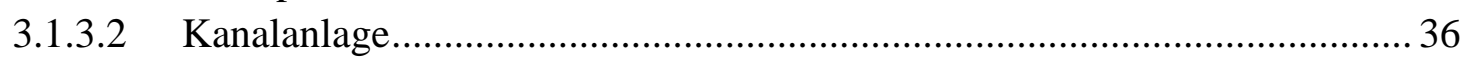

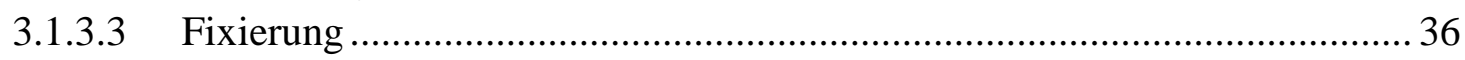

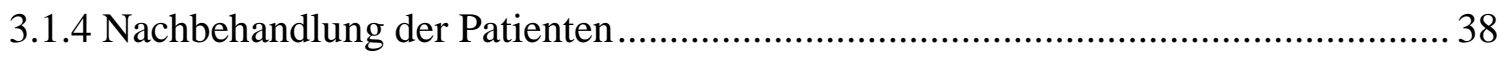

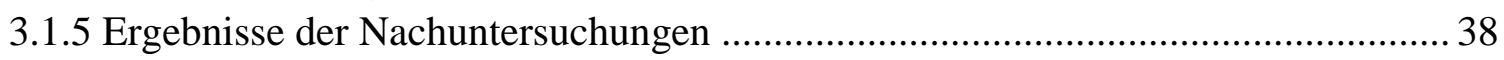

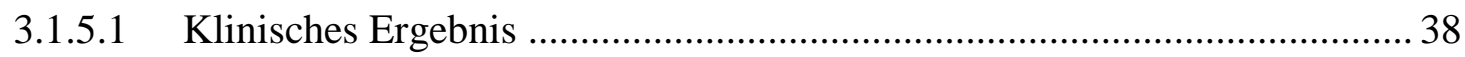

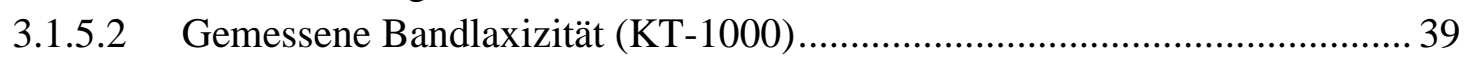

3.1.5.3 Postoperative Beinlängendifferenzen und Achsenabweichungen ................ 40

3.1.5.4 Rerupturen, degenerative Veränderungen und weitere Komplikationen..... 41

3.1.5.5 Operationsergebnisse in Abhängigkeit unterschiedlicher Methoden........... 42

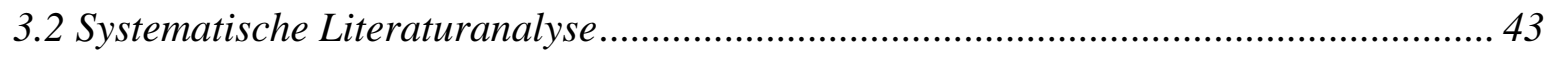

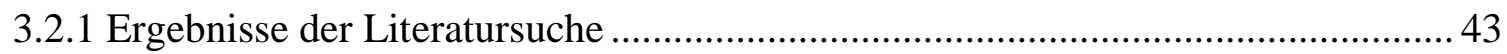

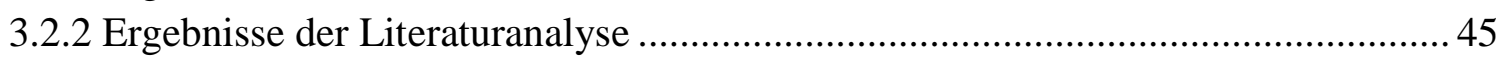

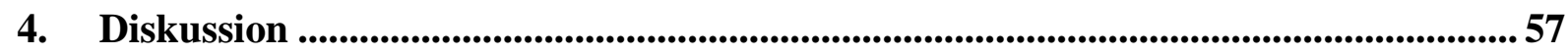

4.1 Beinlängendifferenzen und Achsenabweichungen ....................................................... 58

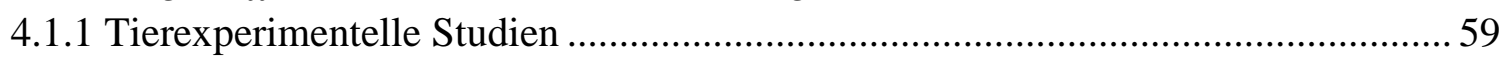

4.1.2 Die Grenzwerte zur Einstufung eines Fehlwachstums....................................... 61

4.1.3 Ursachenanalyse für die Beinlängendifferenzen und Achsenabweichungen ........... 61

4.1.3.1 Mögliche Ursachen für eine Beinlängendifferenz .....................................63 63

4.1.3.2 Mögliche Ursachen für ein Genu recurvatum......................................... 63

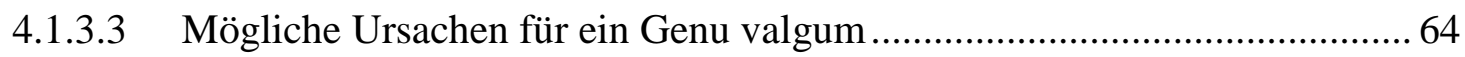

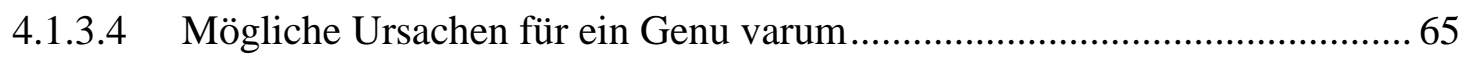

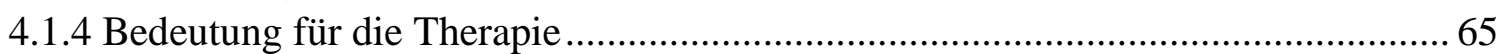

4.2 Können bei Patienten bis zu zwölf Jahren mit einer vorderen Kreuzbandplastik zufriedenstellende Ergebnisse erreicht werden? Erzielen diese Patienten ein schlechteres Therapieergebnis als ältere Patienten mit offenen Wachstumsfugen? .............................. 67

4.3 Ist bei Patienten bis zu zwölf Jahren eine fugenschonende Technik notwendig, oder können die Transplantatkanäle fugenkreuzend angelegt werden? .................................... 69

4.4 Ist eine fugenschonende Operationstechnik bei der vorderen Kreuzbandplastik bei offenen Wachstumsfugen generell nötig oder kann fugenkreuzend operiert werden?........ 70

4.5 Welches Transplantat kann für die Kreuzbandplastik bei Patienten bis zu zwölf Jahren und bei Patienten im Wachstumsalter (altersunabhängig) empfohlen werden?..... 
4.6 Der Einfluss des Zugangsweges zur Anlage des femoralen Knochenkanals auf das Auftreten von Beinlängendifferenzen oder Achsenanbweichungen 75

4.7 Gelenkferne oder gelenknahe Fixierung?.... 75

4.8 Naht der Bandstümpfe bei Kreuzbandverletzungen im Wachstumsalter 76

4.9 Kreuzbandnaht gegenüber Kreuzbandplastik bei offenen Wachstumsfugen .... 77

4.10 Schwächen der Multicenterstudie 77

4.11 Schwächen der Literaturanalyse 78

4.12 Anregungen für zukünftige Studien 79

5. Zusammenfassung 81

6. Anhang 84

6.1 Nachuntersuchungsbogen der Multicenterstudie. 84

6.2 Daten der Multicenterstudie. 88

6.3 Daten der Patienten bis zu zwölf Jahren aus der systematischen Literaturanalyse..... 94 6.4 Beinlängendifferenzen und Achsenabweichungen aus der systematischen Literaturanalyse 96

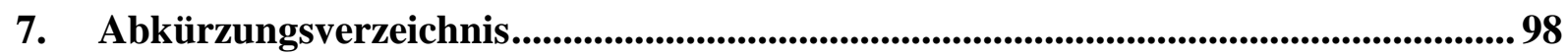

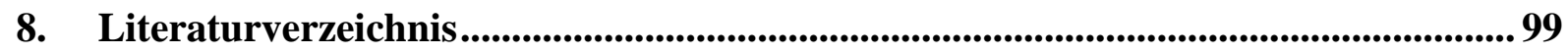

9. Abbildungsverzeichnis ................................................................................................................ 111

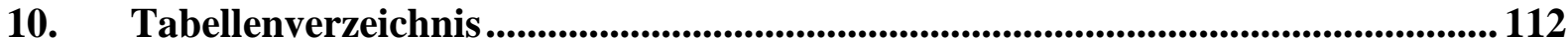




\section{Einleitung}

\subsection{Anatomie und Physiologie des Kniegelenks}

\subsubsection{Anatomie des Kniegelenks}

Das Kniegelenk ist das größte und das komplexeste Gelenk des menschlichen Körpers. Distal verdickt sich das Femur zu zwei mit Gelenkknorpel überzogenen Rollen, den Condyli femoris. Dorsal sind die beiden Kondylen durch die Fossa intercondylaris voneinander getrennt, ventral durch die Facies patellaris, auf der die Kniescheibe gleitet, miteinander verbunden. Die beiden Condyli femoris artikulieren mit dem Condylus medialis und lateralis, den gegenüberliegenden Gelenkflächen der Tibia. Zwischen diesen befindet sich die Eminentia intercondylaris, eine nicht überknorpelte Fläche mit Knochenvorsprüngen (Jakob und Stäubli 1990). Zwischen den stark gekrümmten Femurkondylen und der flacheren Gelenkpfanne des Tibiakopfes vergrößern zwei Menisken als transportable Gelenkflächen die druckübertragende Kontaktfläche. Sie besitzen eine obere und eine untere Gelenkfläche, ihr histologischer Bau ist uneinheitlich. Nahe den Adhäsionsstellen mit Ligamenten bestehen sie aus gut durchblutetem faserigen Bindegewebe, welches dann zuerst in Faserknorpel und ganz innen in reinen hyalinen Knorpel übergeht (Jakob und Stäubli 1990). Beide Menisken sind über Vorder- und Hinterhörner am Tibiaplateau fixiert und über das Ligamentum (Lig.) transversum genus miteinander verbunden. Der mediale Meniskus ist halbmondförmig und größer als der laterale, er ist über das Lig. meniscofemorale mit dem medialen Femurkondylus und über das Lig. meniscotibiale mit der Tibia verwachsen. Der stärker gekrümmte laterale Meniskus hält über das Lig. meniscofemorale anterius und posterius Verbindung zum Femur. Er ist verschiebbarer und wird dadurch bei Verletzungen seltener in Mitleidenschaft gezogen als der mediale Meniskus (Wirth und Zichner 2005). Die Menisken sind außerdem wichtig für das Abfangen von Rotationsbewegungen und an der Propriozeption beteiligt (Müller 1975).

Die umgebende Gelenkkapsel besteht aus zwei Schichten, der Membrana fibrosa und der Membrana synovialis. Die Gelenkkapsel entspringt ventral am Femur, ein bis zwei Zentimeter oberhalb der Knorpelknochengrenze, dorsal an der Linea intercondylaris und am Knorpelrand der Kondylen. Distal ist die Kapsel an der Knorpelknochengrenze mit dem Tibiakopf verwachsen. Ventral ist die Kniescheibe, ein Sesambein der Streckmuskulatur, zusammen mit der zugehörigen Strecksehne, dem Ligamentum patellae, in die Gelenkkapsel eingegliedert. Seitlich neben dem Ligamentum patellae wird die Kapsel durch die Retinacula 
patellae mediale und laterale verstärkt, welche als straffe Bandzüge zum Unterschenkel ziehen. Dorsal sind das Ligamentum popliteum obliquum und arcuatum eingegliedert. Die mit Gleitflüssigkeit gefüllte Gelenkhöhle setzt sich in mehrere Aussackungen, die sogenannten Recessus fort (Wirth und Zichner 2005).

Das Kniegelenk ist ein Gelenk mit Bandführung (Menschik 1974). Der seitliche Bandapparat wird von den beiden Kollateralbändern gebildet. Das Lig. collaterale tibiale (Innenband) und Lig. collaterale fibulare (Außenband) stabilisieren in gestreckter Kniestellung das Kniegelenk insbesondere in der Frontalebene, bei gebeugtem Knie begrenzen sie die Außenrotation (Hertel und Schweiberer 1975). Am Lig. collaterale fibulare kann ein anteriorer, posteriorer und profunder Abschnitt mit unterschiedlichen Funktionen unterschieden werden (Wirth und Zichner 2005), das Lig. collaterale fibulare wirkt zusätzlich synergistisch zum hinteren Kreuzband (Jakob und Stäubli 1990).

Anterolateral wird das Kniegelenk durch den Tractus iliotibialis verstärkt, dieses Sicherungssystem wird als Lig. femorotibiale laterale anterius bezeichnet. Dorsal wird das Kniegelenk durch die Sehnen der hier verlaufenden Muskeln, durch die oben genannten Ligamenta popliteum arcuatum und obliquum, sowie durch den Musculus popliteus stabilisiert, welcher synergistisch zum hinteren Kreuzband wirkt (Wirth und Zichner 2005).

Den sogenannten Zentralpfeiler des Kniegelenkes bilden die beiden Kreuzbänder. Das Ligamentum cruciatum anterius (siehe unten) und das Ligamentum cruciatum posterius befinden sich zwischen den Femurkondylen und den beiden Schichten der Gelenkkapsel. Sie liegen also innerhalb der fibrösen Gelenkkapsel, aber außerhalb der von Synovia ausgekleideten Gelenkhöhle (Wirth und Zichner 2005). Die Kreuzbänder dienen dem Zusammenhalt der Gelenkkörper. Bei einer Außenrotation des Unterschenkels wickeln sich die überkreuzten Bänder voneinander ab und entspannen sich. Bei einer Innenrotation wickeln sie sich umeinander, spannen sich an und begrenzen dadurch diese Bewegung. Bei Überstreckung des Beines sind beide Bänder angespannt (Wagner und Schabus 1982).

Am Kniegelenk befinden sich mehrere Schleimbeutel. Diese sogenannten Bursae synoviales setzten die Reibung an den gefährdeten Stellen herab. Von besonderer Bedeutung ist die Bursa suprapatellaris, da sie eine Verbindung zur Gelenkhöhle hat. Unter der Kniescheibe liegt das Corpus adiposum infrapatellare, ein Fettkörper, der sich der Verformung des Gelenkes entsprechend anpassen kann. 


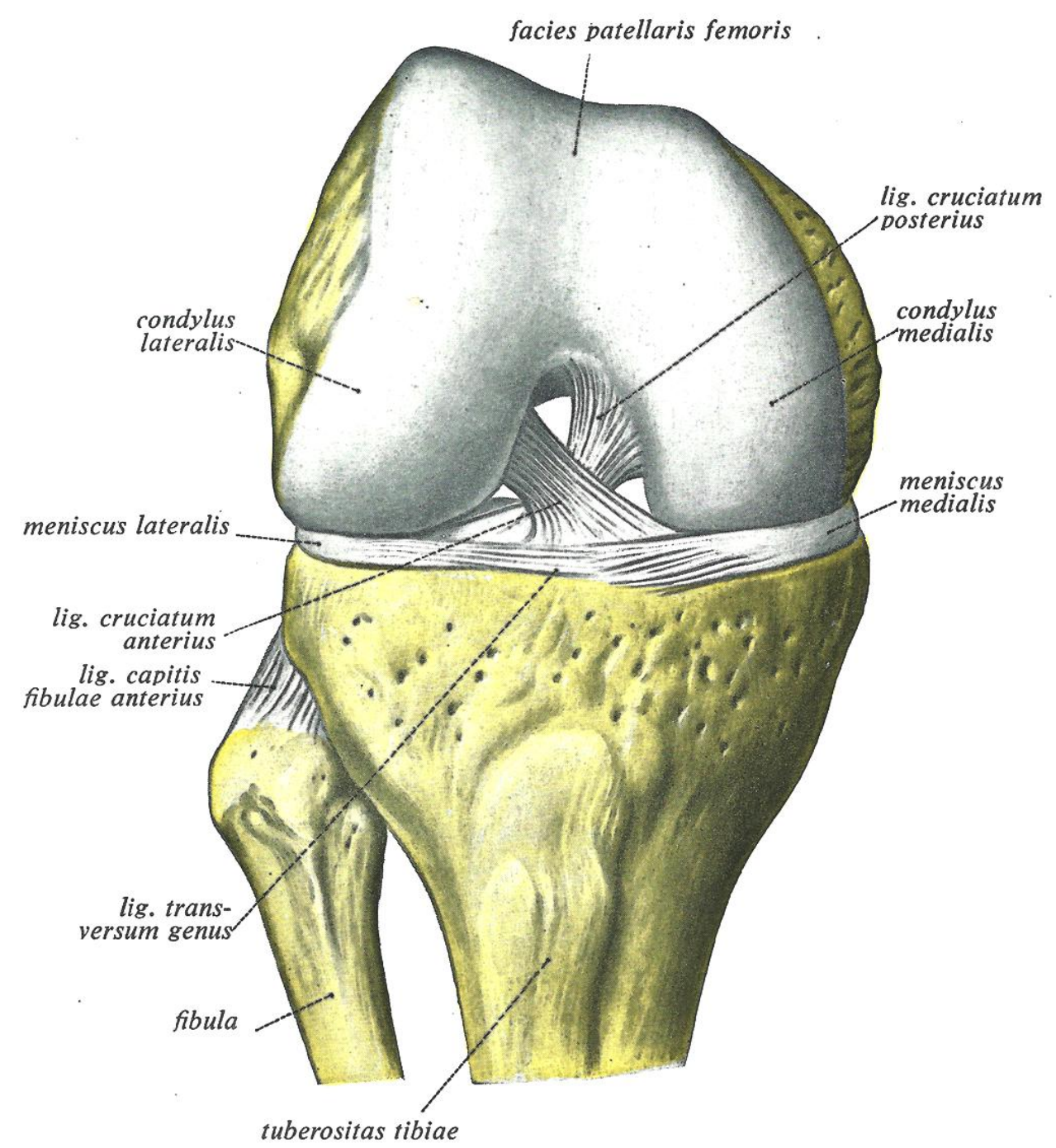

Abbildung 1: Rechtes Kniegelenk eines Erwachsenen, in gebeugter Stellung nach Entfernung der Gelenkkapsel und der Seitenbänder. Aus: Sobotta und Becher (1972), S. 127. Mit freundlicher Genehmigung des Elsevier-Verlags.

Im Gegensatz zum Erwachsenen besitzt das kindliche Kniegelenk drei Wachstumsfugen, die sich alle in der Nähe des Vorderen Kreuzbands (VKB) befinden und daher für die Therapie einer VKB-Ruptur von großer Bedeutung sind. Man unterscheidet eine distale femorale sowie proximale tibiale Wachstumsfuge und die Apophyse der Tuberositas tibiae. Diese Fugen besitzen das höchste Wachstumspotential des kindlichen Körpers, zwei Drittel des Längenwachstums der unteren Extremität finden hier statt. Bei Mädchen verschließen sich die Fugen in einem Alter von etwa 14 bis 15 Jahren, bei Jungen etwa zwei Jahre später (Seil und Robert 2005). 


\subsubsection{Anatomie des vorderen Kreuzbandes}

Das VKB ist eine der ligamentären Verbindungen zwischen Femur und Tibia. Proximal inseriert es an der posterioren medialen Fläche des lateralen Femurkondylus. Dieser Ansatz des VKB befindet sich in direkter räumlicher Nähe zu der distalen femoralen Wachstumsfuge. Die Distanz beträgt nur $3 \mathrm{~mm}$ und bleibt über die gesamte Wachstumsphase konstant (Seil 2002).

Distal setzt das VKB an der Tibia an der Area intercondylaris anterior an. Die Verlaufsrichtung des VKB bei gestrecktem Kniegelenk ist also von dorsal, lateral, kranial nach ventral, medial und kaudal. Das Band als Ganzes besteht aus einem anteromedialen und einem posterolateralen Bündel, die sich wiederum aus einzelnen Fasern zusammensetzen, welche parallel angeordnet sind und in einer leichten spiralförmigen Auswärtsrotation verlaufen (Dodds und Arnoczky 1994).

Bei gestrecktem Kniegelenk stehen alle Fasern des VKB unter Spannung. In gebeugter Stellung sind nur die anterioren Fasern gespannt, die posterior gelegenen verlaufen entspannt (Dodds und Arnoczky 1994; Friederich et al. 1992; Girgis et al. 1975). Odensten und Gillquist (1985) geben die Länge des VKB beim Erwachsenen mit durchschnittlich $31 \mathrm{~mm}$ an. Als maximale Reißkraft des VKB beim Erwachsenen werden in der Literatur Werte zwischen 1725 Newton und 2160 Newton angegeben (Wirth und Zichner 2005).

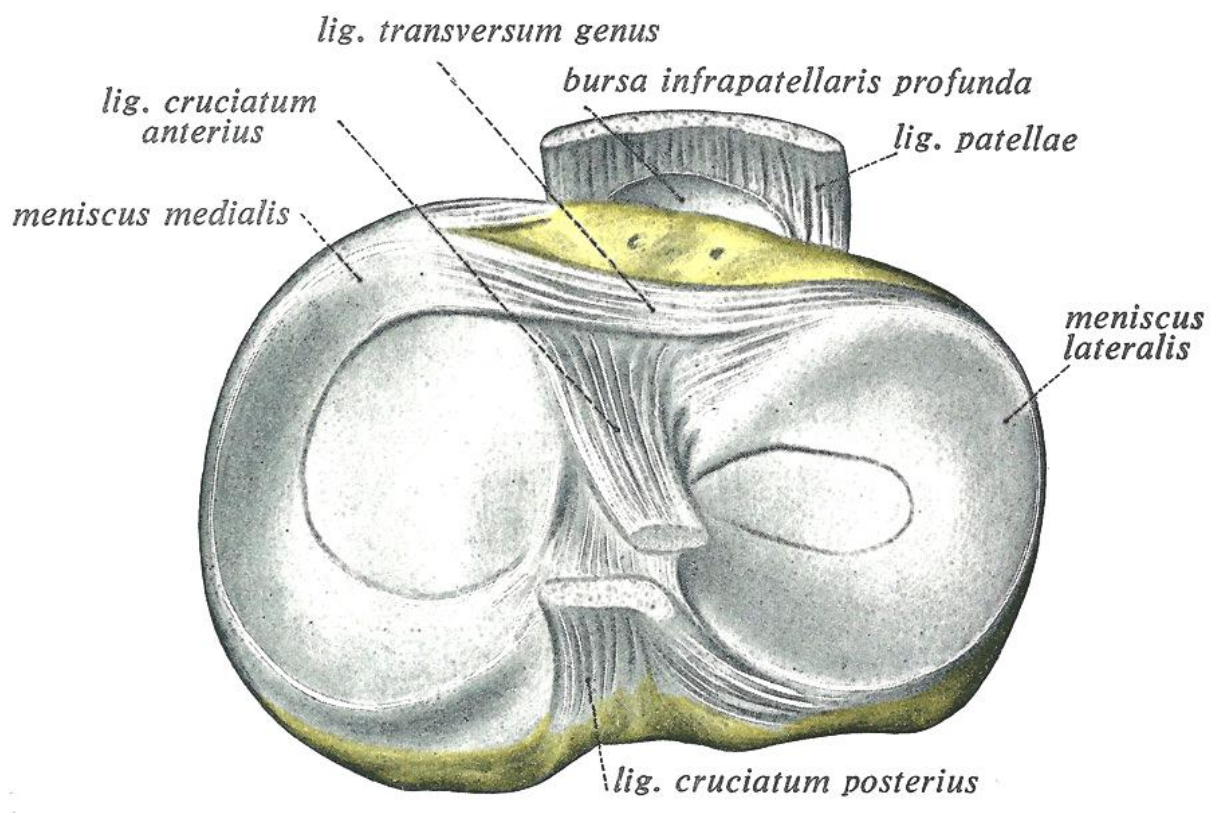

Abbildung 2: Kondylen der Tibia mit den Menisken und den Ursprüngen der beiden Kreuzbänder. Aus: Sobotta und Becher (1972), S. 127. Mit freundlicher Genehmigung des Elsevier-Verlags. 


\subsubsection{Biomechanik des Kniegelenks}

Das Kniegelenk verbindet obere und untere Funktionseinheit des Beines. Einerseits bildet das Kniegelenk in Streckstellung zusammen mit Femur und Tibia eine feste Tragesäule. Andererseits erweitert es das Bewegungsfeld des Fußes in Beugestellung, in der auch Drehbewegungen möglich sind. Beugung und Streckung für die Fortbewegung stehen im Vordergrund der Funktion.

Das Kniegelenk ist also ein Drehscharniergelenk, ein sogenannter Trochoginglymus. Die Roll-Gleit-Bewegungen, sowie das physiologische Gelenkspiel werden durch die sechs Freiheitsgrade des Kniegelenks ermöglicht. Unterschieden werden drei Translations(anterior-posterior, medial-lateral, Kompression-Distraktion) und drei Rotationsfreiheitsgrade (Flexion-Extension, Außenrotation-Innenrotation, Abduktion-Adduktion) (Jakob und Stäubli 1990; Siliski 1994). Viele dieser Gelenkbewegungen sind aneinander gekoppelt. Der normale Bewegungsumfang der Knieextension und Flexion beträgt ausgehend von der Neutral- oder Nullstellung 10-0-145 Grad (Jakob und Stäubli 1990). Die Überstreckung von zehn Grad ist bei gleichzeitiger Außenrotation des Unterschenkels um fünf Grad möglich, diesen Mechanismus bezeichnet man als Schlussrotation. Eine weitere Überstreckung wird vom dorsalen Teil des Bandapparats verhindert. Die passive Beugung ist bis zum Anstoßen der Ferse am Gesäß möglich und damit größer als die aktive Beugung durch die Beinmuskulatur. $\mathrm{Zu}$ Beginn der Beugebewegung aus voller Streckung rollen die Femurkondylen ohne Gleitbewegung auf dem Tibiaplateau, die mediale Seite während der ersten zwölf bis fünfzehn Grad Beugung, die laterale Seite während der ersten zwanzig Grad. Bei weiterer Beugung findet zusätzlich zu der Rollbewegung eine Gleitbewegung der Femurkondylen nach ventral statt, bei maximaler Beugung gleiten sie ohne eine Rollbewegung auf dem Tibiaplateau (Kapandji 1970). Während der Flexion verschieben sich die Menisken nach dorsal. Die Beugung bedingt auch eine Bewegung im Femoropatellargelenk. In gestrecktem Zustand befindet sich die Kniescheibe auf der Bursa suprapatellaris und liegt nur mit ihrer Unterkannte der Gelenkfläche des Femur auf. Während der Flexion des Knies wandert die Kniescheibe 5 bis $7 \mathrm{~cm}$ auf dem Femur nach kaudal, wobei der Anpressdruck zwischen der Facies patellaris des Femurs und der Facies articularis der Patella zunimmt (Wagner und Schabus 1982).

In Extension werden Rotationsbewegungen durch die straffen Seitenbänder verhindert. Mit steigender Beugung nimmt die Rotationsmöglichkeit zu. Sie ist am größten, wenn das Knie etwa 130 Grad gebeugt ist. Die Innenrotation ist in wesentlich geringerem Umfang möglich 
als die Außenrotation, da sie durch die sich aufwickelnden Kreuzbänder gehemmt wird. Bei 20 Grad gebeugtem Kniegelenk beträgt die Innen- bzw. Außenrotation 15-0-35 Grad (Jakob und Stäubli 1990).

\subsubsection{Funktion des vorderen Kreuzbandes}

Das anteromediale Bündel des vorderen Kreuzbandes begrenzt die sogenannte vordere Schublade, also die Translationsbewegung des Tibiaplateaus nach vorne relativ zu den Femurkondylen (Cabaud 1983; Petersen und Zantop 2007; Piziali et al. 1980). Es wird in dieser Funktion durch die Menisken als Sekundärstabilisatoren unterstützt (Papageorgiou et al. 2001), jedoch bringt das VKB bezüglich der Begrenzung der vorderen Schublade alleine einen Anteil von 86 Prozent des Widerstandes des gesamten Kapsel-Bandapparats des Knies auf (Butler et al. 1980). Das posterolaterale Bündel des VKB sichert die Rotationsstabilität, insbesondere bei gestrecktem Gelenk (Petersen und Zantop 2007). Auch übernimmt das vordere Kreuzband eine Rolle in der Stabilisierung bei Valgus- und Varusbelastung (Inoue et al. 1987; Piziali et al. 1980). Zusätzlich zu den mechanischen Funktionen spielt das VKB auch eine Rolle als Sensor und ist somit Teil des neurophysiologischen Regelkreises, da es Mechanorezeptoren enthält und sich über die Propriozeption an der Steuerung der Motorik des Beines beteiligt (Haus und Halata 1990; Schutte et al. 1987). Das vordere Kreuzband übernimmt somit eine Kernfunktion der Kniegelenkskinematik (Müller 1977).

\subsubsection{Achsenabweichungen des Kniegelenks}

Die Tragachse ergibt sich aus der Verbindungslinie zwischen den Mittelpunkten des Hüftund des oberen Sprunggelenkes. Beim Genu rectum, also einer physiologischen Stellung der Skelettteile zueinander, verläuft die Tragachse minimal medial der Kniegelenksmitte (Strecker 2006). Bei einer unphysiologischen Achsenausrichtung in der Frontalebene spricht man von einer Varus- bzw. Valgusabweichung, in der Sagittalebene von Ante- bzw. Rekurvation. Bei einem Genu varum (O-Bein) ist die Tragachse nach medial verschoben. Als Genu valgum (X-Bein) bezeichnet man eine Fehlstellung, bei der die Tragachse lateral des Kniegelenkzentrums verläuft (Sharma et al. 2010). Beim Genu recurvatum ist die Tragachse nach ventral des Kniegelenkzentrums verschoben. Ist das Bein ohne Verschieben der Tibia gegenüber dem Femur über fünfzehn Grad im Wachstumsalter bzw. zehn Grad nach Wachstumsabschluss überstreckbar, spricht man von einem echten Genu recurvatum (Westhoff et al. 2007). 
Bei einem gesunden Kind oder Jugendlichen besteht grundsätzlich eine knöcherne Symmetrie der Kniegelenke in allen räumlichen Dimensionen im Rechts-LinksSeitenvergleich. Im Falle einer einseitigen Fehlstellung wird die kontralaterale Seite als Referenz verwendet (Strecker und Keppler 2002).

Es werden anatomische und mechanische Achsen an der unteren Extremität unterschieden, die anatomischen Achsen verlaufen im Zentrum der Diaphysen des Femurs und der Tibia, die mechanischen Achsen verbinden die Zentren der jeweiligen angrenzenden Gelenke. Zur Quantifizierung der Varus- bzw. Valgusabweichung wird der anatomische oder mechanische laterale distale Femurwinkel und der mediale proximale Tibiawinkel bestimmt (Strecker 2006). Der anatomische bzw. mechanische laterale distale Femurwinkel ist der Winkel zwischen anatomischer bzw. mechanischer Femurachse und der femoralen Kniebasislinie, einer Verbindungslinie der distalen Konturen der Femurkondylen, er beträgt 79 bis 83 bzw. 85 bis 90 Grad. Da die anatomische Tibiaachse mit der mechanischen nahezu identisch ist, wird nicht zwischen anatomischem bzw. mechanischem medialen proximalen Tibiawinkel unterschieden. Der mediale proximale Tibiawinkel wird durch Tibiaachse und tibiale Kniebasislinie, einer Verbindungslinie der proximalen Konturen der Tibiakondylen, gebildet und beträgt 85 bis 90 Grad. Zur Berechnung der Ante- bzw. Rekurvation werden die anatomischen Achsen verwendet, es werden der anatomische dorsale distale Femurwinkel (79 bis $87 \mathrm{Grad}$ ) und der anatomische dorsale proximale Tibiawinkel (81 bis 86 Grad) berechnet (Strecker 2006).

\subsection{Die vordere Kreuzbandruptur}

Die vordere Kreuzbandruptur ist eine typische Sportverletzung. Am häufigsten findet sich die Verletzung bei Sportarten, die sehr schnelle Richtungswechsel, also Beschleunigungs- und Abbremsbewegungen in Kombination mit Drehbewegungen, verlangen. Besonders prädisponierende Sportarten sind unter anderem Fußball, Volleyball, Tennis, Basketball und Skifahren (Schmidt-Wiethoff und Dargel 2007). Zum Zerreißen des Bandes führt am häufigsten eine Kombination aus Flexion, Abduktion und Außenrotation im Kniegelenk bei Fixierung von Fuß und Unterschenkel (Petersen et al. 2005). Typische Unfallmechanismen sind auch ein Flexions-Varus-Innenrotationstrauma und eine Hyperextension des Kniegelenks (Strobel et al. 1995). Meistens wird von den betroffenen Patienten ein deutlich fühlbares Zerreißen, manchmal sogar von einem hörbaren Knallen im Kniegelenk, während des Traumas berichtet. Dieses geht typischerweise mit plötzlichen Schmerzen des Knies und mit 
einem sehr schnellen Anschwellen des Gelenks einher. Häufig fühlt sich das Knie sofort instabil an (Fehnel und Johnson 2000). In über 50 Prozent der Fälle geht ein gerissenes Kreuzband mit einer zusätzlichen Meniskusverletzung einher (Fehnel und Johnson 2000). Auch die Kollateralbänder und der Gelenkknorpel sind häufig mitbetroffen.

Diagnostiziert wird die Kreuzbandruptur anhand der Anamnese, der klinischen Untersuchung, einschließlich verschiedener Stabilitätstests und der Magnetresonanztomographie. Die gängigsten Stabilitätstests sind der Lachman-Test, mit einer Sensitivität von 85 und Spezifität von 94 Prozent, der hoch spezifische (98 Prozent) aber wenig sensitive (24 Prozent) PivotShift-Test und der vordere Schubladentest, welcher bei einer älteren Verletzung eine gute Sensitivität (92 Prozent) und Spezifität (91 Prozent) aufweist, jedoch bei akuten Verletzungen des VKB weniger verlässliche Informationen liefert (Benjaminse et al. 2006). Die Magnetresonanztomographie kann eine VKB-Ruptur mit 96 prozentiger Sensitivität und Spezifität nachweisen (Friedman und Jackson 1996; Kocabey et al. 2004).

\subsection{Verletzungsmuster der vorderen Kreuzbandruptur im Wachstumsalter}

Die Verletzungsmuster des Kniegelenkes bei Patienten im Wachstumsalter unterscheiden sich deutlich von denen im Erwachsenenalter. Kommt es beim Erwachsenen von allen Bändern des Kniegelenks am häufigsten zu einer Ruptur des VKB, so ist diese Verletzung bei Patienten im Wachstumsalter sehr selten. Von allen vorderen Kreuzbandrissen macht der Anteil von VKB-Rupturen im Wachstumsalter nur 0,5 bis 3 Prozent aus (Andrish 2001; Lipscomb und Anderson 1986).

Unter den Rupturen des VKB im Wachstumsalter differenziert man zwischen knöchernen Ausrissen des Bandes bzw. Frakturen der Eminentia intercondylaris einerseits und den ligamentären Rupturen andererseits. Bei kleinen Kindern mit unreifem Skelett und einem Reifestadium Tanner eins oder zwei, überwiegen die knöchernen Ausrisse, da der Knochen der Eminentia intercondylaris relativ $\mathrm{zu}$ dem vorderen Kreuzband eine geringere Reißfestigkeit besitzt (Rinaldi und Mazzarella 1980). Mit zunehmendem Alter und Reife des Skeletts nehmen die ligamentären Rupturen anteilsmäßig zu (Arndt et al. 1999; Kellenberger und von Laer 1990). Es wird angenommen, dass auch die Energie des Traumas Einfluss auf das Verletzungsmuster hat. Hochgeschwindigkeitstraumata verursachen vermutlich eher intraligamentäre Rupturen, niederrasante Traumen hingegen knöcherne Ausrisse (Skak et al. 1987). 
Bei den ligamentären Rupturen handelt es sich in zwei Dritteln der Fälle um vollständige Rupturen. Von diesen sind wiederum zwei Drittel intraligamentäre Risse, in zehn Prozent ist das VKB vom distalen Ansatz, in 15 Prozent vom proximalen Ansatz abgerissen (Seil 2002). Nach Meyers und McKeever (1959 und 1970) werden die knöchernen Ausrisse in drei Typen unterteilt. Bei Typ I ist das knöcherne Fragment gar nicht oder kaum disloziert. Bei Typ II ist es großteils disloziert, jedoch nicht über die ganze Länge, und bei Typ III ist das ausgerissene Knochenfragment vollständig disloziert. Bei Typ III+ liegt zusätzlich eine Rotationskomponente vor. Zu dieser Klassifikation wurde von Zaricznyj (1977) noch ein Typ IV hinzugefügt, bei welchem mehrere knöcherne Fragmente vorliegen.

Die Therapie bei einem knöchernen Bandausriss ist wie bei Erwachsenen auch bei Kindern und Jugendlichen weitgehend standardisiert (Seil 2002). Bei Typ-I- und Typ-II-Läsionen ist die Therapie meist konservativ. Bei Ausrissfrakturen vom Typ III und IV ist eine operative Versorgung in offener oder arthroskopischer Technik Standard (Lo et al. 1998; Mah et al. 1996).

In den letzten Jahren kam es immer häufiger zu Berichten von VKB-Rupturen im Wachstumsalter (Lo et al. 1998; Moksnes et al. 2008; Steadman et al. 2006). Dieser Trend ist vermutlich darauf zurückzuführen, dass in jüngerer Vergangenheit erstens vermehrt auch immer jüngere Menschen Leistungssport und Risikosportarten betreiben, dass zweitens die untersuchenden Kliniker ein gewachsenes Bewusstsein für diese Verletzung entwickelt haben und dass sich drittens die diagnostischen Methoden wie die Arthroskopie und die Magnetresonanztomografie verbessert haben (Steadman et al. 2006).

Die vorliegende Arbeit beschäftigt sich mit ligamentären Rupturen des VKB im Wachstumsalter.

\subsection{Therapiemöglichkeiten der ligamentären vorderen Kreuzbandruptur im Wachstumsalter}

\subsubsection{Besonderheiten, die sich für die Therapie bei Vorliegen offener Wachstumsfugen ergeben}

Das Ziel der Behandlung einer VKB-Ruptur ist die Wiederherstellung eines biomechanisch stabilen Kniegelenks, mit welchem der Patient ohne Einschränkungen leben und Sport treiben kann. Bei erwachsenen Patienten ist die Therapie einer VKB-Ruptur überwiegend standardisiert. Praktiziert wird eine Operation, bei der das Band durch eine intraartikuläre Bandplastik unter Verwendung knöcherner Bohrkanäle ersetzt wird. Laut einer Untersuchung 
in den USA wird dieses Verfahren von nahezu 100 Prozent der Operateure verwendet (Delay et al. 2001).

Auf das Knie eines wachsenden Skeletts übertragen, bedeutet dieses Operationsverfahren jedoch ein Vorgehen, bei welchem die Bohrkanäle mit dem darin verlaufenden Transplantat die distale Wachstumsfuge des Femurs und die proximale Wachstumsfuge der Tibia perforieren (Seil 2002).

Es wird allerdings angenommen, dass von jeder Beschädigung der Wachstumsfugen ein potentielles Risiko für eine Wachstumsstörung ausgeht (Seil 2002; Stadelmaier et al. 1995). Wird die Wachstumsfuge an ihrem Rand, dem perichondralen Ring oder innerhalb der Fuge verletzt, zum Beispiel durch eine transepiphysäre Bohrung, so kann dies an der betroffenen Stelle zu einer knöchernen Durchbauung der Wachstumsfuge führen (Seil 2002). Man nennt diese Durchbauung eine Epiphysiodese. Kleine Epiphysiodesebrücken können eigenständig wieder resorbiert oder gesprengt werden und müssen das Knochenwachstum nicht zwangsläufig beeinträchtigen (Seil und Robert 2005). Epiphysiodesebrücken mit einem größeren Durchmesser können jedoch zu einem vollständigen Wachstumsstopp oder einem asymmetrischen Restwachstum mit einer axialen Fehlstellung des Beines führen (Seil 2002). Der Grenzwert für die tolerierte Defektgröße ist noch nicht ausreichend bekannt (Bonnel et al. 1984; Pous et al. 1979). Form und Ausmaß der Deformität hängen von der Lage der Epiphysiodesebrücke und dem verbleibenden Wachstum ab. Bei randständig gelegener Überbrückung resultiert ein asymmetrisches Wachstum mit einer Achsenabweichung, bei einer zentralen Knochenbrücke kommt es eher $\mathrm{zu}$ einer symmetrischen Wachstumsverminderung mit einer Verkürzung des betroffenen Beines im Vergleich zur Gegenseite (Seil 2002).

Eine Durchbohrung der Wachstumsfuge im Rahmen einer Kreuzbandrekonstruktion muss also nicht zwangsläufig bleibende Schäden verursachen. Dennoch führte die Gefahr einer solchen Wachstumsstörung in der Vergangenheit $\mathrm{zu}$ einer großen Zurückhaltung der Operateure gegenüber der Durchführung solcher Eingriffe (Micheli und Foster 1993).

Im Wachstumsalter werden wegen dieser Problematik, im Gegensatz zum Vorgehen bei Erwachsenen, auch weiterhin noch Operationsmethoden praktiziert, die bei der Therapie der Erwachsenen aufgrund ihrer schlechten Ergebnisse nicht mehr verwendet werden. Dazu zählen hauptsächlich die konservative Therapie, die Kreuzbandnaht und fugenschonende Kreuzbandplastiken, also solche Techniken, welche die Wachstumsfuge umgehen und unversehrt belassen. Jedoch werden auch fugenkreuzende Operationstechniken, welche mehr 
oder weniger dem Vorgehen beim erwachsenen Patienten entsprechen, trotz der beschriebenen Risiken angewandt (Seil 2002).

\subsubsection{Konservative Therapie}

Die Ergebnisse einer konservativen Behandlung von VKB-Rupturen bei Erwachsenen sind mehrheitlich schlecht. Die Behandlung führt auf längere Sicht meist zu chronischer Instabilität des Knies mit daraus resultierenden Meniskusverletzungen, zu Knorpelschäden, Schmerzen und Arthrose des Kniegelenkes (Schachter und Rokito 2007). Daher wird die konservative Behandlung bei Erwachsenen nur noch in Ausnahmefällen empfohlen.

Einerseits aus Hoffnung, dass die Selbstheilung bei Patienten im Wachstumsalter zu besseren Ergebnissen führt und andererseits aus Angst vor chirurgischen Eingriffen und deren Risiken für das wachsende Skelett, wurden viele Patienten im Wachstumsalter trotz des Wissens um die schlechten Ergebnisse bei Erwachsenen konservativ behandelt (Seil 2002).

Eine solche Behandlung schließt Gipsruhigstellung oder Orthesenbehandlung, Physiotherapie und eine Begrenzung der sportlichen Aktivitäten ein. Die Physiotherapie hat zum Ziel, die stabilisierende Muskulatur zu kräftigen und die Beweglichkeit zu erhalten bzw. zu verbessern. Den jungen Patienten wird geraten, belastende Sportarten wie beispielsweise Fußball, Basketball, Skifahren oder Turnen zu vermeiden. Offensichtlicher Vorteil dieser Behandlungsmethode ist, dass sie völlig ohne invasiven Eingriff auskommt und damit alle mit einer Operation verbundenen Risiken, wie Narkosekomplikationen, Infektionen oder Verletzungen der Wachstumsfugen mit daraus resultierenden Wachstumsstörungen ausschließt.

Leider hat sich gezeigt, dass die Ergebnisse bei einer konservativen Behandlung im Wachstumsalter ebenso wie beim Erwachsenen mit einer schlechten Prognose behaftet sind (Mizuta et al. 1995; Scavenius et al. 1999; Schachter und Rokito 2007). Besonders die hohe Rate an Meniskusläsionen (bis zu 39 Prozent) (Scavenius et al. 1999) und degenerativen Veränderungen (Mizuta et al. 1995) haben dazu geführt, dass heute kaum noch konservativ behandelt wird. Eine Ausnahme stellen Fälle dar, in denen die konservative Behandlung der zeitlichen Überbrückung bis nach dem Wachstumsabschluss dient, um anschließend mit einer Kreuzbandersatzoperation ein stabiles Kniegelenk herzustellen (Schachter und Rokito 2007). 


\subsubsection{Kreuzbandnaht}

Eine Form der operativen Versorgung der VKB-Ruptur ist die Naht des Bandes. Hierbei werden die beiden Kreuzbandstümpfe wieder aneinander adaptiert und in diesem Zustand durch Fadenmaterial gesichert. Die Intention dieses Verfahrens ist es, durch die Adaptation die körpereigene Heilung des Bandes zu ermöglichen und wieder ein stabiles natürliches VKB herzustellen. Vorteil dieser Methode gegenüber einer Kreuzbandplastik ist, dass sie ohne Transplantat auskommt und daher Komplikationen durch die Entnahme des körpereigenen Transplantats umgeht. Auch das Risiko von Wachstumsstörungen ist minimal, da keine oder nur sehr dünne Bohrkanäle notwendig sind. Als Nahttechnik wird überwiegend die Naht nach Marshall verwendet (Marshall et al. 1979). Die Ergebnisse der Kreuzbandnaht wurden bisher nur in wenigen Studien untersucht (Arndt et al. 1999; DeLee und Curtis 1983; Seil 2002).

\subsubsection{Kreuzbandplastik}

\subsubsection{Fugenschonende Techniken}

Es gibt viele unterschiedliche Methoden, wie das VKB ersetzt werden kann, ohne dass dabei die Wachstumsfugen perforiert werden. Grundsätzlich werden extraartikuläre von intraartikulären Verfahren unterschieden. Früher wurden diese Techniken auch bei erwachsenen Patienten angewandt, aufgrund schlechter Ergebnisse jedoch bald aufgegeben (Seil 2002).

Bei den extraartikulären Verfahren befindet sich das Transplantat außerhalb der Gelenkkapsel.

Die intraartikulären Verfahren sind vielfältig. Unterteilen kann man sie wiederum in solche, bei denen das Transplantat extraepiphysär, und solche, bei denen das Transplantat transepiphysär verläuft. Bei extraepiphysärem Verlauf wird das Transplantat um die Wachstumsfuge herumgeführt und metaphysär, also jenseits der Fuge, befestigt (Seil 2002). Die Befestigung des Transplantats kann entweder in einem knöchernen Bohrkanal erfolgen, welcher metaphysär fugenschonend platziert wird (Robert und Bonnard 1999) oder das Transplantat kann außen am metaphysären Knochen befestigt werden (Janarv et al. 1996; Micheli et al. 1999).

Bei einer epiphysären Transplantatführung werden die Bohrkanäle gelenknah durch den Knochen geführt. Der Kanal befindet sich vollständig in der Epiphyse und die 
Wachstumsfuge wird nicht durchbohrt (Lipscomb und Anderson 1986). Dieses Operationsverfahren ist anspruchsvoll, denn der Bohrkanal verläuft nahezu parallel zu der Epiphysenfuge. Bei einer nur leichten Abweichung der Bohrrichtung kann die Fuge tangiert werden, was bedeutet, dass dieser auf die Fläche bezogen ein sehr großer Schaden zugefügt werden kann (Seil 2002).

Ein grundsätzlicher Nachteil der fugenschonenden Operationsmethoden ist, dass das Transplantat nur schwerlich isometrisch, also genau wie das ursprüngliche VKB, platziert werden kann (Liddle et al. 2008; Vaquero et al. 2005). Im Gegenzug merken manche Autoren an, dass die Gefahr einer vorzeitigen Fugenverknöcherung im Bereich der Bohrung und somit das Risiko einer Beinlängendifferenz oder Achsenabweichung mit diesen Methoden verringert werden soll (Cohen et al. 2009; Seil 2002).

\subsubsection{Fugenkreuzende Techniken}

Das Verfahren der wachstumsfugenkreuzenden Bandplastik ist nahezu identisch mit dem, das standardmäßig bei erwachsenen Patienten durchgeführt wird (Schachter und Rokito 2007). Hier verläuft der Bohrkanal in Femur und Tibia von dem ursprünglichen Kreuzbandansatz durch Epiphyse sowie Metaphyse und kreuzt somit die dazwischen liegende Wachstumszone. Femoral liegt der Kanal auf Höhe der Wachstumsfuge exzentrisch posterolateral, tibial wird die Fuge relativ zentral gekreuzt. Im Falle eines Fugenverschlusses käme es femoral also zu einem asymmetrischen Restwachstum mit Achsenabweichung, tibial eher zu einem symmetrischen Wachstumsstopp (Seil 2002).

Die fugenkreuzenden Operationsmethoden bergen das grundsätzliche Risiko einer Stimulation oder Hemmung der Aktivität der Wachstumsfuge und damit das Risiko eines knöchernen Fehlwachstums mit Achsenabweichung oder Beinlängendifferenz (Vaquero et al. 2005).

\subsubsection{Partiell fugenkreuzende Techniken}

Die Verfahren der partiell fugenkreuzenden Techniken vereinen die fugenschonenden mit den fugenkreuzenden Operationsmethoden. Je nach Verfahren werden entweder nur die femorale oder nur die tibiale Wachstumsfuge perforiert (Andrews et al. 1994; Hoffmann et al. 1998; Lipscomb und Anderson 1986). 


\subsection{Fragestellung und Ziel}

Wie im vorausgehenden Teil geschildert, wird bei Patienten mit offenen Wachstumsfugen derzeit eine Vielzahl verschiedener operativer Methoden zur Versorgung einer vorderen Kreuzbandruptur angewandt. Dies beruht darauf, dass aktuell bezüglich des für Patienten im Wachstumsalter am besten geeigneten Verfahrens noch eine Unsicherheit und Uneinigkeit unter den Chirurgen besteht (Henry et al. 2009; Schachter und Rokito 2007; Vaquero et al. 2005). Bis heute hat sich noch keines der möglichen Verfahren als den anderen überlegen herauskristallisiert (Cohen et al. 2009; Lukas et al. 2007).

In der Literatur gehen die Ansichten diesbezüglich weit auseinander. So sind Lukas et al. (2007) der Meinung, dass sich ein fugenkreuzendes Implantat aufgrund des Risikos einer konsekutiven Wachstumsstörung grundsätzlich verbietet. Kocher et al. (2007) hingegen schreiben, dass eine fugenkreuzende Rekonstruktion zu exzellenten Ergebnissen führt, wobei die Revisionsrate sehr niedrig und die Gefahr einer Wachstumsstörung minimal ist.

Neben der Technik, mit der das Transplantat eingebracht wird, sind auch dessen Fixierung und die Art des Transplantats selbst noch Gegenstand der Diskussion (Henry et al. 2009; Lukas et al. 2007; Schachter und Rokito 2007).

Ziel dieser Arbeit ist es, zum einen im Rahmen einer Multicenterstudie die Behandlungsverfahren und Therapieergebnisse mehrerer deutscher unfallchirurgischer Kliniken bezüglich Patienten bis zu zwölf Jahren mit VKB-Ruptur und offenen Wachstumsfugen $\mathrm{zu}$ erfassen und klinisch $\mathrm{zu}$ evaluieren. Zum anderen werden in einer systematischen Literaturanalyse die publizierten Studien zu Therapien der VKB-Ruptur bei Patienten mit offenen Wachstumsfugen und deren Ergebnissen untersucht. Hierbei sollen auch insbesondere Patienten bis zum zwölften Lebensjahr berücksichtigt werden.

\section{Folgende Fragen sollen mithilfe der Multicenterstudie und der systematischen Literaturanalyse für Patienten mit einer vorderen Kreuzbandruptur geklärt werden:}

1. Wie häufig sind Beinlängendifferenzen und Achsenabweichungen bei Patienten bis zu zwölf Jahren nach VKB-Plastik? Sind Beinlängendifferenzen und Achsenabweichungen in dieser Altersgruppe häufiger als bei älteren Patienten? Welche Ursachen liegen ihnen zugrunde und wie können sie vermieden werden?

2. Können bei Patienten bis zu zwölf Jahren mit einer vorderen Kreuzbandplastik zufriedenstellende Ergebnisse erzielt werden? 
3. Erzielen die bis zu zwölfjährigen Patienten bei einem Kreuzbandersatz ein schlechteres Therapieergebnis als ältere Patienten mit offenen Wachstumsfugen?

4. Ist bei Patienten im Wachstumsalter und speziell bei Patienten bis zu zwölf Jahren eine fugenschonende Technik notwendig oder können die Transplantatkanäle fugenkreuzend angelegt werden?

5. Welches Transplantat ist bei diesen Patienten am besten geeignet?

6. Kann der femorale Kanal über das anteromediale Portal platziert werden, oder sollte man diesen besser transtibial anlegen?

7. Wie hoch ist die Rerupturrate nach operativer Versorgung einer VKB-Ruptur?

\section{Zusätzlich soll die systematische Literaturanalyse der Klärung folgender Fragen dienen:}

1. Ist bei der Kreuzbandplastik eine gelenknahe oder gelenkferne Fixierung von Vorteil?

2. Lassen sich mit der Kreuzbandplastik bei Patienten mit offenen Wachstumsfugen bessere Ergebnisse erzielen als mit der Kreuzbandnaht?

3. Ist die Naht der Bandstümpfe bei Patienten bis zwölf Jahren erfolgversprechender als bei Patienten mit einem Alter ab 13 Jahren?

Auf die Untersuchung der Wachstumsstörungen, deren Ursachen und mögliche Vermeidung soll in dieser Arbeit ein Hauptaugenmerk gelegt werden. 


\section{Material und Methode}

\subsection{Multicenterstudie}

\subsubsection{Studienart}

Die retrospektive nationale Multicenterstudie wurde durch die zuständige Ethikkomission geprüft und bewilligt. Bis zum Juni 2011 wurden Patienten an zehn verschiedenen Kliniken rekrutiert und einmalig anhand eines standardisierten Protokolls nachuntersucht.

Die Eltern der Patienten, sowie die Patienten selbst, wurden ausführlich über den Sinn und Zweck der Studie unterrichtet. Eine Einverständniserklärung zur Teilnahme an der Studie wurde von allen Patienten bzw. deren Erziehungsberechtigten schriftlich eingeholt.

\subsubsection{Teilnehmende Kliniken}

Patienten aus folgenden Kliniken wurden in die Studie eingeschlossen:

- Marienkrankenhaus Kaiserswerth

- Friederikenstift Hannover

- Medizinische Hochschule Hannover

- Sportklinik Stuttgart

- Katholisches Klinikum Koblenz

- Martin-Luther-Krankenhaus Berlin

- Universitätsklinikum Gießen

- Universitätsklinikum Göttingen

- ARTICO Sportklinik Villingen-Schwenningen

- Universitätsklinik Freiburg.

\subsubsection{Informationsübermittlung über die Homepage www.kreuzband-kind.de}

Um den an der Multicenterstudie teilnehmenden Kliniken einen schnellen Überblick über die Studie sowie deren Verlauf zu ermöglichen, wurde eine passwortgeschützte Internetseite mit der Webadresse www.kreuzband-kind.de erstellt.

Hier konnten von den teilnehmenden Kliniken Studieninformationen, relevante Literatur und aktuelle Ergebnisse eingesehen werden. Außerdem konnten die Aufklärungs- und Nachuntersuchungsbögen heruntergeladen werden. 


\subsubsection{Patientenauswahl}

Als Einschlusskriterien zur Aufnahme in die Multicenterstudie wurden definiert:

- Patienten bis zu einem Alter von einschließlich zwölf Jahren zum Zeitpunkt der Operation

- Patienten mit ligamentärer vorderer Kreuzbandruptur

- Operative Versorgung der VKB-Ruptur

- Nachuntersuchung frühestens sechs Monate nach der Operation.

Zum Ausschluss aus der Studie führte:

- Knöcherner Kreuzbandausriss

- Alter über zwölf Jahre

- Zusätzliche hintere Kreuzbandruptur.

\subsubsection{Datenerfassung}

Alle Patienten wurden einmalig anhand eines standardisierten Protokolls nachuntersucht.

Nachfolgend ein Überblick über die erhobenen Daten. Der Untersuchungsbogen selbst befindet sich im Anhang.

\section{Individuelle Daten}

- Geburtsdatum

- Geschlecht

- Unfalldatum

- Unfallhergang

- OP-Datum

- Nachuntersuchungsdatum

- Größe und Gewicht präoperativ

- Größe und Gewicht zum Nachuntersuchungstermin

- Betroffene Seite.

\section{Operative Daten}

- Verwendetes Transplantat

- Fixierung femoral und tibial

- Lage des Bohrkanals femoral und tibial in Bezug auf die Wachstumsfuge (fugenkreuzend oder fugenschonend) 
- Zugangsweg zur Anlage des femoralen Kanals (transtibial oder über das anteromediale Portal)

- Evaluation der Bohrkanalanlage

- Wurde ein Handbohrer benutzt?

- Bohrkanaldurchmesser femoral und tibial.

\section{Begleitverletzungen}

- Art der Begleitverletzungen

- Therapie der Begleitverletzungen.

\section{Nachbehandlung}

- Wie lange durfte nur teilbelastet werden?

- Wurde eine Orthese verordnet?

Wenn ja, wie lange?

- Wurde Krankengymnastik verordnet?

Wenn ja, wie lange?

- Dauer der verordneten Sportpause

Wie lange wurde die Sportpause tatsächlich eingehalten?

\section{Nachuntersuchung}

- Beinlängendifferenz zum Zeitpunkt der Nachuntersuchung im Vergleich zur präoperativ gemessenen Beinlängendifferenz

- Varusabweichung

- Valgusabweichung

- Genu recurvatum

- Andere klinische Fehlstellungen

- Degenerative Veränderungen

- Auffälligkeiten im konventionellen Röntgen in zwei Ebenen (Nur wenn Röntgenaufnahme unabhängig von der Studie bereits vorhanden war)

- Stabilitätsmessung durch Messung der Bandlaxizität des neuen VKB, in Form eines instrumentellen Lachmann-Tests mithilfe des KT-1000 Arthrometers für das rechte sowie das linke Knie (wenn Messgerät vorhanden)

- Kam es zu einer Reruptur des Transplantats? 
- Sonstige Komplikationen

- Lysholm-Score

- $\quad$ IKDC 2000.

\subsubsection{Instrumenteller Lachman-Test mit dem KT-1000-Arthrometer}

1982 wurde das KT-1000-Kniebandarthrometer vorgestellt, ein Instrument zur objektiven Messung der Verschiebbarkeit der Tibia relativ zum Femur in der Sagittalebene (Wroble et al. 1990). Zur Messung liegt der Patient auf dem Rücken, das Knie befindet sich in einer 20- bis 35-Grad-Flexionsstellung. Das Gerät wird mit zwei Bändern an der Vorderseite des Unterschenkels befestigt und am Gelenkspalt ausgerichtet, über einen Griff am Arthrometer kann ein standardisierter Zug bzw. Druck auf die Tibia ausgeübt werden. Zwei frei bewegliche Sensoren sind auf der Patella und dem Tuberculum tibiae platziert und messen relative anterior-posterior Bewegungen zwischen beiden Punkten (Daniel et al. 1985a; Daniel et al. 1985b).

Der KT-1000 gilt als exaktes und verlässliches Verfahren zur Diagnose einer vorderen Knieinstabilität (Boyer et al. 2004; Jardin et al. 1999; Tyler et al. 1999).

\subsubsection{Lysholm-Score}

Der Lysholm-Score (siehe Abb. im Anhang) ist ein Evaluationssystem, welches von Lysholm und Gillquist (1982) veröffentlicht wurde. Es handelt sich um eine Methode der subjektiven Zustandsbewertung des Knies. Vom Patienten werden acht verschiedene Symptome bewertet (Hinken, Gehhilfen, Blockierung, Instabilität, Schmerzen, Schwellung, Treppensteigen, tiefe Hocke). Besonders stark gewichtet werden die Symptome Schmerz und Schwellung. Auf diese Weise kann eine Gesamtzahl von bis zu 100 Punkten erreicht werden. Ein Ergebnis von 95 bis 100 Punkten gilt als sehr gut, von 84 bis 94 als gut, 65 bis 83 als mäßig und kleiner als 65 als schlecht.

\subsubsection{IKDC knee examination score}

1987 wurde der Internationale Ausschuss zur Dokumentation von Knieverletzungen (International Knee Documentation Committee) gegründet. Dieser entwickelte den knee examination score, der meist als IKDC-Score oder einfach IKDC bezeichnet wird, als neues Bewertungsschema für Kniebandverletzungen (Hefti et al. 1993). Der Score gewann unter den vielen verschiedenen weltweit verwendeten Bewertungsschemata schnell an Bedeutung 
(Risberg et al. 1999). Es handelt sich um einen überwiegend objektiven Score, welcher in verschiedene Kategorien unterteilt ist. Jedes einzelne untersuchte Merkmal kann mit A (normal), B (fast normal), C (abnormal) oder D (deutlich abnormal) bewertet werden. Die schlechteste Bewertung innerhalb einer Kategorie ergibt das Ergebnis für die ganze Kategorie. Ebenso verhält es sich mit der Gesamtevaluation, hier bestimmt das schlechteste Ergebnis einer Kategorie das Gesamtergebnis (Hefti et al. 1993).

Bei dem in der Multicenterstudie verwendeten IKDC-Score (IKDC 2000) handelt es sich um eine modernisierte Form des Bewertungsschemas (siehe Abb. im Anhang).

\subsection{Systematische Literaturanalyse}

\subsubsection{Literaturrecherche}

\subsubsection{Datenbanken}

Durchsucht wurden die Literaturdatenbank Medline, die Cochrane Library, die SpringerVerlagsdatenbank, Thieme-Verlagsdatenbank, sowie die Literaturdatenbank Embase (Excerpta Medica Database)

Die letzte Aktualisierung der Suche in allen genannten Datenquellen erfolgte am 17. Juli 2008.

Medline (Akronym für Medical Literature Online) gilt als die international anerkannte Referenz (Kunz R et al. 2000; Sackett et al. 2000). Trotzdem berichteten Dickersin et al. (1994) im British Medical Journal, dass je nach Fragestellung nur zwischen 30 bis 80 Prozent aller bekannten und veröffentlichten Studien mittels Medline $\mathrm{zu}$ finden sind. Medline entstammt der medizinischen Bibliothek der Vereinigten Staaten (National Library of Medicine, NLM) und legt den Schwerpunkt auf angloamerikanische Literatur. Bei einem täglichen Aktualisierungsintervall beinhaltet sie Zeitschriftenartikel von 1950 bis heute, aktuell sind etwa 20.000.000 Nachweise aus 4.800 internationalen biomedizinischen Fachzeitschriften verzeichnet. Über PubMed ist ein kostenloser Zugriff auf Medline möglich, auf Abstracts kann in etwa 76 Prozent der Fälle zugegriffen werden.

Die Cochrane Library ist eine Einrichtung der Cochrane Collaboration, sie bietet ca. 600.000 systematische Übersichtsarbeiten und kontrollierte klinische Studien aus einem Zeitraum von 1948 bis heute, eine Aktualisierung wird vierteljährlich vorgenommen. 
Die Springer-Verlagsdatenbank ist eine Online-Bibliothek für Wissenschaft, Technik und Medizin des Springer-Verlags, welcher den größten Fachinformationsanbieter im deutschsprachigen Raum darstellt. 90 Prozent der Publikationen aus ungefähr 2.200 Wissenschafts- und Fachzeitschriften erscheinen in englischer Sprache. Ein kostenpflichtiger Zugang zu den Volltexten als PDF-Dokument ist möglich.

Die Thieme-Verlagsdatenbank enthält etwa 84.000 Artikel aus 180 wissenschaftlichen und medizinischen Zeitschriften des Thieme Verlags ab 1981. Eine Aktualisierung wird täglich vorgenommen. Die Volltexte können kostenpflichtig direkt als PDF-Datei abgerufen werden. Embase (Excerpta Medica Database) enthält Humanmedizinische Publikationen der Excerpta Medica Referatezeitschriften. Der Umfang beträgt etwa 25.000.000 Artikel aus 7000 internationalen Zeitschriften, der Schwerpunkt liegt auf europäischen Veröffentlichungen. Enthalten sind Literaturnachweise ab 1947, der Bestand wird täglich aktualisiert.

Die „Publikations-Bias“ bezeichnet das Phänomen, dass signifikante und somit erwünschte Forschungsergebnisse häufiger publiziert werden als nicht signifikante Ergebnisse. Englischsprachige Artikel sind gegenüber anderssprachigen überrepräsentiert. Diese Tatsache wird als „Sprachen-Bias“ bezeichnet.

\subsubsection{Suchbegriffe}

Folgende Suchbegriffe wurden in den verschiedenen Suchmaschinen verwendet:

- anterior cruciate ligament

- $A C L$

- child

- immature

- pediatric

- young

- open growth plates

- growth age

- open physes.

Als sinnvolle Kombination der einzelnen Begriffe erwies sich folgende Suchformel: 
(anterior cruciate ligament OR ACL) AND (child OR immature OR pediatric OR young OR open growth plates OR growth age OR open physes).

\subsubsection{Auswahl der Publikationen}

Von allen unter der angewendeten Suchformel gelisteten Publikationen wurde als erstes der Titel gelesen. Konnte eine Relevanz der Publikation anhand des Titels nicht sicher ausgeschlossen werden, so wurde anschließend die Zusammenfassung (Abstract) der Publikation gelesen, sofern diese verfügbar war.

In die Literaturanalyse aufgenommen wurden Publikationen zur operativen Therapie von ligamentären vorderen Kreuzbandrupturen im Wachstumsalter, die gleichzeitig folgende Einund Ausschlusskriterien erfüllten:

\section{Einschlusskriterien}

Folgende drei Kriterien mussten von der Publikation erfüllt werden, um in den Datenpool mit einbezogen zu werden.

1. A) Alter des Patienten bis zu zwölf Jahren bzw. B) Offene Wachstumsfugen des distalen Femur und der proximalen Tibia zum Operationszeitpunkt.

2. Behandlung der Ruptur des vorderen Kreuzbandes mit einer Kreuzbandplastik oder einer Naht der Kreuzbandstümpfe.

3. Klinische Nachuntersuchung, Kontrolle des OP-Erfolgs.

\section{Ausschusskriterien}

Die Erfüllung eines oder mehrerer der folgenden Kriterien führten zum Ausschluss des jeweiligen Artikels aus der Studie.

1. Rein konservative Behandlung.

2. Refixierung femoraler oder tibialer knöcherner Ausrisse der vorderen Kreuzbänder.

3. Beschreibung mehrerer Behandlungsstrategien (zum Beispiel teilweise konservative Behandlung und außerdem Behandlung mit Kreuzbandplastik oder -naht) und eine Ergebnisbeschreibung, die es nicht zulässt, spezifische Ergebnisse für die verschiedenen Behandlungsmethoden zu differenzieren.

4. Darstellung einer OP-Technik, ohne Beschreibung von operierten Patienten und deren Behandlungsergebnissen. 
5. Reine Expertenmeinung eines Autors oder Diskussion mehrerer Autoren bezüglich der Kreuzbandruptur im Wachstumsalter ohne die Beschreibung eigener Patienten und Behandlungen.

6. Review-Artikel.

Es wurde bewusst keine Mindestdauer für den Zeitraum der Patientennachuntersuchung festgelegt. Mit dem Nichtvorhandensein eines derartigen Ausschlusskriteriums sollte es ermöglicht werden, auch jene Artikel mit auswerten zu können, welche keine Angaben zu dem Nachuntersuchungszeitraum machen.

Auch wurden Publikationen mit eingeschlossen, in welchen die Behandlung und die Behandlungsergebnisse von rekonstruierten Kreuzbandrupturen zwar beschrieben werden, jedoch nicht das Hauptaugenmerk des Artikels darstellen. Bei der Auswertung dieser "nebenbei" erwähnten Daten wurden an diese jedoch die gleichen Anforderungen wie an alle anderen gestellt. Es wurden nicht nur Publikationen aufgenommen deren gesamtes Patientenkollektiv den Ein- und Ausschlusskriterien entsprach, sondern auch solche, in denen nur ein Teil der Patienten diese Kriterien erfüllte. In diesen Fällen wurden dann nur die Daten der entsprechenden Patienten ausgewertet.

Es wurde keine Mindestzahl des Patientenkollektivs formuliert, auch Einzelfallberichte wurden mit einbezogen.

Ein knöcherner Ausriss des Kreuzbandes, der durch eine Refixation behandelt wurde, wurde nicht in die Auswertung mit aufgenommen (siehe Ausschlusskriterien). Sollte ein knöcherner Ausriss jedoch therapiert werden, indem das Kreuzband, wie bei einem ligamentären Riss üblich, durch eine Ersatzplastik versorgt wurde, so wurde dieser Fall auch dementsprechend ausgewertet.

Alle eingeschlossenen Publikationen wurden in voller Länge gelesen. Zur Vervollständigung und Überprüfung des Ergebnisses der durchgeführten Literatursuche wurden die Quellenangaben sämtlicher eingeschlossener Artikel durchgesehen. Veröffentlichungen, die noch nicht erfasst worden waren, wurden ergänzend nachbestellt. 


\subsubsection{Datenerfassung}

Alle unten aufgeführten Daten wurden getrennt für die zwei untersuchten Patientengruppen erfasst. Zum einen für die kleinere Gruppe der Patienten mit einem Alter von bis zu zwölf Jahren, zum anderen für die größere Gruppe aller Patienten mit offenen Wachstumsfugen.

Alle eingeschlossenen Artikel wurden auf folgende Daten überprüft:

- Anzahl der operativen Versorgungen insgesamt

Patienten bei denen gleichzeitig das rechte und das linke vordere Kreuzband rupturiert waren und therapiert wurden, wurden als zwei eigenständige Fälle gewertet.

- Anzahl der Kreuzbandersatzplastiken

- Anzahl der Kreuzbandnähte

Als Kreuzbandnähte wurden sowohl Nähte der zwei verbliebenen Kreuzbandstümpfe, als auch Reinsertionen von femoral- oder tibialseitigen knochennahen Bandabrissen gewertet. Traumatische Bandelongationen, die operativ gerafft bzw. verkürzt wurden, wurden auch als Kreuzbandnähte gezählt.

- Durchschnittsalter des Patientenkollektivs zum Zeitpunkt der Operation

- Art der Operationsmethode der Kreuzbandersatzplastiken in Bezug auf die distale femorale sowie die proximale tibiale Wachstumsfuge

Die Einordnung der Art der Operationsmethode erfolgte anhand einer entsprechenden Beschreibung im Text oder, falls nicht beschrieben, wenn möglich anhand eines abgebildeten postoperativen Röntgenbildes. Als fugenkreuzend galt jede Art der Kreuzbandplastik, bei der der Bohrkanal für das Transplantat durch die Fuge geführt wurde. Unter fugenschonend wurden sowohl intraartikuläre Bandersatzplastiken, welche die Wachstumsfuge nicht perforierten, als auch extraartikuläre Bandersatzplastiken, gelistet.

○ Anzahl der femoral und tibial fugenkreuzenden Plastiken

- Anzahl der femoral und tibial fugenschonenden Plastiken

- Art der verwendeten Transplantate

○ Patellasehne (Ligamentum patellae)

Dieses Transplantat wird auch mit BTB als Abkürzung von bonetendon-bone bezeichnet. $\mathrm{Zu}$ beachten ist, dass die Patellasehne oft auch nur mit Periostschlauch ohne Knochenblöcke verwendet wurde.

○ Sehne des Musculus gracilis und/oder des Musculus semitendinosus

(meist als Hamstring bezeichnet)

- Sehne des Musculus quadriceps 
- Fascia lata / iliotibial Band

(Diese Begriffe werden synonym verwendet)

- Allograft

- Fixierung: Anzahl gelenknaher und gelenkferner Fixierungen der Transplantate

In den Fällen in denen kein Knochenkanal gebohrt wurde, galt eine Befestigung des Transplantats auf der dem Gelenkspalt abgewandten Seite der Wachstumsfuge als „gelenkfern“, auf der dem Gelenkspalt zugewandten Seite als „gelenknah“. Wurde der Bandersatz durch einen knöchernen Kanal geführt, wurde eine Fixierung im Kanal auf der dem Gelenkspalt abgewandten Seite oder vom Gelenkspalt aus gesehen jenseits des Kanals als gelenkfern gewertet. Eine Fixierung im Kanal auf der dem Gelenkspalt zugewandten Seite wurde als gelenknah eingestuft. Auch ohne diesbezügliche Beschreibung war in einigen Fällen ein Rückschluss vom verwendeten Fixationsmaterial auf die Art der Fixierung möglich. Eine Fixierung mit einer Interferenzschraube oder eine Transfixation mit quer durch den Kanal gelegtem Befestigungsmaterial wurde als gelenknahe Fixierung eingestuft, wenn keine anders lautende Beschreibung oder widersprechende Röntgenaufnahmen vorlagen. Transplantate welche mit einem Knopf oder in der over the top position befestigt waren oder Hamstringplastiken, bei welchen der Ansatz der verwendeten Sehnen auf der distalen Seite erhalten wurde, wurden als gelenkfern fixiert gewertet.

- Anzahl der VKB-Plastiken, welche femoral und tibial gelenknah fixiert wurden

- Anzahl der VKB-Plastiken, welche femoral und tibial gelenkfern fixiert wurden

- Zugangsweg zur Anlage des femoralen Knochenkanals

Bei Nichtvorhandensein einer genauen Beschreibung wurde von abgebildeten Röntgenaufnahmen die Operationsmethode abgelesen. Über den Winkel, mit dem der Kanal im Knochen liegend $\mathrm{zu}$ erkennen ist, kann auf den Zugangsweg rückgeschlossen werden.

- Anzahl der Patienten, bei denen der femorale Kanal über das anteromediale Portal platziert wurde

- Anzahl der Patienten, bei denen der femorale Kanal transtibial, also als direkte Verlängerung des tibialen Knochenkanals, gebohrt wurde

- Operationsergebnis / Ergebnisse der letzten Patientennachuntersuchung

○ Durchschnittlicher Lysholm-Score (siehe unten)

- Anzahl der Patienten, bei denen es innerhalb des Beobachtungszeitraums erneut zu einer Ruptur des genähten oder ersetzten Kreuzbandes kam

Hierbei wurde nicht unterschieden ob vom Autor angegeben wurde, dass die Reruptur auf ein adäquates Trauma folgte oder nicht. Als keine Reruptur wurde gewertet, wenn beschrieben war, dass keine Rerupturen auftraten, oder wenn die Anzahl erneuter Bandrupturen nicht erwähnt wurde, so dass stillschweigend davon ausgegangen wurde, dass keine 
Reruptur vorlag. Eine Reruptur wurde also nur als solche gewertet, wenn diese vom Autor beschrieben wurde. Ein Rückschluss von einem als schlecht beschriebenen klinischen Ergebnis oder einer sehr hohen Bandlaxizität auf eine Ruptur des Bandes wurde nicht gezogen. Partielle Rerupturen wurden wie komplette Rerupturen gewertet.

- Beinlängendifferenz oder Achsenabweichung

Eine Beinlängendifferenz von $10 \mathrm{~mm}$ oder mehr, oder eine Achsenabweichung zwischen Femur und Tibia im Vergleich zur Gegenseite von drei Grad oder mehr, wurde als Beinlängendifferenz oder Achsabweichung definiert. Andere Arten von Wachstumsstörungen wurden nicht gewertet. Eine angegebene Beinlängendifferenz oder Achsabweichung, die diese Grenzen überschritt, wurde von uns als Beinlängendifferenz oder Achsenabweichung gewertet, unabhängig davon, ob der Autor des Artikels die Abweichung als Fehlwachstum einstufte oder nicht. Eine von einem Autor beschriebene Beinlängendifferenz oder Achsenabweichung wurde von uns als solche eingestuft, auch wenn nicht das genaue Ausmaß der Längen- oder Winkelabweichung angegeben wurde. Für eine Patientengruppe wurde keine Beinlängendifferenz oder Achsenabweichung gewertet, wenn beschrieben war, dass keine solche auftrat und ebenso, wenn diese Komplikation in dem Artikel gar nicht erwähnt wurde. Wenn Angaben von Beinlängendifferenzen oder Achsenabweichungen in einem Artikel in Form von Durchschnittswerten oder Spannweiten für mehrere Patienten gleichzeitig angegeben wurden, konnten diese nicht ausgewertet werden.

Da die Grenzen für die Bewertung als Fehlwachstum von den verschiedenen Autoren sehr unterschiedlich streng festgesetzt sind, wurden hier für die systematische Literaturanalyse vergleichbar niedrige Grenzwerte gewählt, um alle beschriebenen Fälle einer Beinlängendifferenz oder Achsenabweichung erfassen und auswerten zu können.

○ Bei wie vielen Patienten trat eine Beinlängendifferenz oder eine Achsenabweichung auf?

○ Welcher Art war die beschriebene Beinlängendifferenz oder Achsenabweichung? (Beinlängendifferenz, Genu valgum, Genu varum, Genu recurvatum)

○ Welches Ausmaß hatte die gemessene Beinlängendifferenz oder Achsenabweichung?

(Beinlängendifferenz in Millimeter, Achsabweichung in Grad)

○ Welcher Grund wurde vom Autor für das Auftreten der Beinlängendifferenz oder Achsenabweichung angegeben?

- Evidenzstärke der Publikationen nach Levels of Evidence des Oxford-Centre for Evidence Based Medicine 
Tabelle 1: Levels of Evidence für Therapie- und Präventionsstudien (modifiziert nach Oxford Centre 2009, http://www.cebm.net/index.aspx?o=1025)

\begin{tabular}{|l|l|}
\hline Evidenzlevel & Studienart \\
\hline Ia & Systematischer Review (mit Homogenität) von randomisiert-kontrollierten Studien \\
\hline Ib & Einzelne randomisiert-kontrollierte Studie (mit engem Konfidenzintervall) \\
\hline Ic & $*$ \\
\hline IIa & Systematischer Review (mit Homogenität) von Kohortenstudien \\
\hline IIb & $\begin{array}{l}\text { Einzelne Kohortenstudie (eingeschlossene randomisiert-kontrollierte Studie mit } \\
\text { schlechter Qualität; z.B. <80\% Nachbeobachtungsrate) }\end{array}$ \\
\hline IIc & Ergebnisforschung; ökologische Studien \\
\hline IIIa & Systematischer Review (mit Homogenität) von Fall-Kontroll-Studien \\
\hline IIIb & Einzelne Fall-Kontroll-Studie \\
\hline IV & Fallserie (und qualitative schlechte Kohorten- und Fall-Kontroll-Studien) \\
\hline V & $\begin{array}{l}\text { Expertenmeinung ohne kritische Analyse oder basiert auf physiologischer oder } \\
\text { experimenteller Forschung oder "Grundprinzipien" }\end{array}$ \\
\hline
\end{tabular}

* Trifft zu, wenn alle Patienten starben, bevor die Therapie verfügbar war und nach Einführung der Therapie einige überleben; oder wenn einige Patienten starben, bevor die Therapie verfügbar war und keiner nach Einführung der Therapie stirbt.

Die den Artikeln entnommenen Daten wurden in eine entsprechend konzipierte Excel-Tabelle eingetragen.

Wann immer in einem Artikel zu den untersuchten Daten keine Aussage gemacht wurde, so wurde dieser Punkt mit der Eintragung "keine Angabe" festgehalten. Nicht exakte Angaben wie zum Beispiel "alle fünf Patienten waren jünger als 15 Jahre" oder "zwölfmal wurde die Patellasehne oder die Faszia Lata als Bandersatz verwendet" wurden nicht verwertet. Auch solche Fälle führten zur Eintragung von "keine Angabe" im entsprechenden Feld.

Sollten in ein und demselben Artikel sich widersprechende Angaben gemacht werden, so wurden jene Daten übernommen, welche genauer (z.B. mit Kommastellen) beschrieben waren oder sich öfters innerhalb des Artikels wiederholten.

Bei fehlenden Angaben zu der Operationstechnik wurde, falls dies möglich war, von abgebildeten Röntgenaufnahmen auf die Operationstechnik rückgeschlossen. 


\subsubsection{Berechnung der untersuchten Größen aus den einzelnen Artikeln.}

Aufgrund der Heterogenität der verschiedenen Publikationen ist es nicht möglich, jede Publikation zur Berechnung jeder untersuchten Größe heranzuziehen. Die Berechnung einer Durchschnittsgröße kann immer nur auf Basis eines gewissen Anteils aller in die Literaturanalyse eingeschlossenen Artikel erfolgen, da jeweils nur ein Teil aller Artikel eine Aussage zu dieser untersuchten Größe macht. Daher basieren verschiedene berechnete Größen auf unterschiedlichen zugrunde liegenden Artikelgruppen. Im Ergebnisteil dieser Arbeit sind zu jeder berechneten Größe die entsprechenden Artikel angegeben. 


\section{Ergebnisse}

\subsection{Multicenterstudie}

\subsubsection{Kliniken und Patienten}

In zehn Kliniken wurden 25 Patienten nachuntersucht. Beteiligt waren das Marienkrankenhaus Kaiserswerth mit sieben, das Katholische Klinikum Koblenz sowie die ARTICO Sportklinik Villingen-Schwenningen mit jeweils drei, die Sportklinik Stuttgart, das Martin-Luther-Krankenhaus Berlin, die Medizinische Hochschule Hannover und die Universitätskliniken Göttingen und Freiburg mit jeweils zwei Patienten, sowie das Krankenhaus Friederikenstift Hannover und die Universitätsklinik Gießen mit jeweils einem Patienten. Zwei Patienten hatten sich ihre Verletzung während des Schulsports zugezogen, 17 während der Ausübung einer Sportart außerhalb der Schule und zwei bei einem Sturz mit dem Fahrrad. Für vier Patienten war der Unfallhergang nicht beschrieben worden. 16-mal war das linke VKB betroffen, neunmal das rechte. Das Durchschnittsalter der 16 Jungen und neun Mädchen betrug zum Zeitpunkt der Operation 10,4 (7 bis 12) Jahre. Die Zeitspanne die zwischen Unfall und Operation lag erstreckte sich zwischen sieben und 305 Tagen, wobei der Durchschnitt bei 83,5 Tagen lag. Für acht Patienten war dieses Zeitintervall nicht angegeben. Die Patienten wurden durchschnittlich 603,8 (231 bis 2247) Tage nach der Operation wieder einbestellt und nachuntersucht. Zwischen dem Zeitpunkt der Operation und der Nachuntersuchung betrug das durchschnittliche Längenwachstum der Patienten 9,0 (0 bis 46) $\mathrm{cm}$, für sechs Patienten wurde es nicht angegeben. Die durchschnittliche Gewichtszunahme betrug in dieser Zeit 6,7 (1 bis 30) kg, wobei hier für neun Patienten keine Angabe gemacht wurde. 


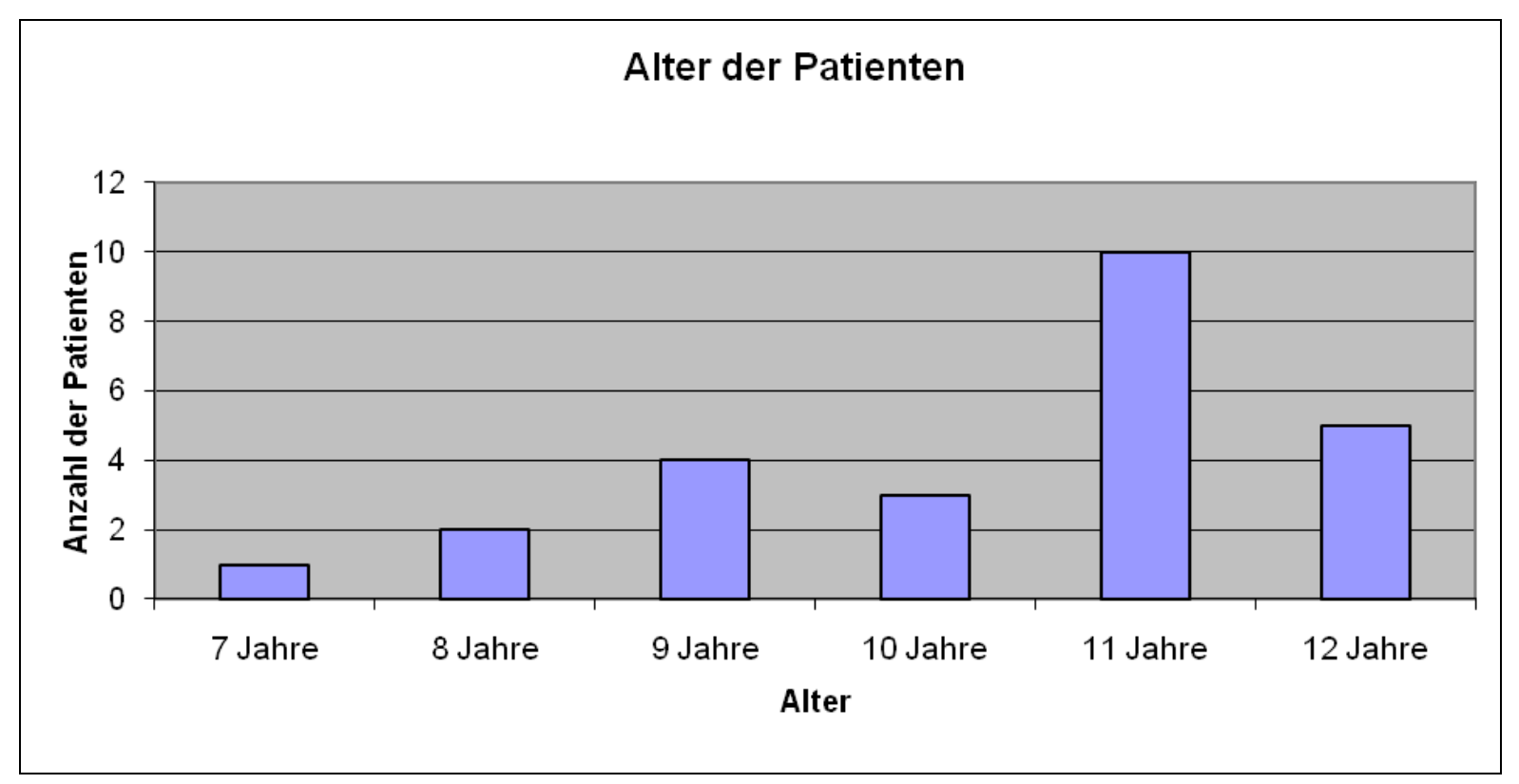

\section{Abbildung 3: Alter der Patienten}

\subsubsection{Begleitverletzungen}

Neun der 25 Patienten hatten zusätzlich zu der Ruptur des VKB weitere Pathologien des betroffenen Kniegelenkes. Zwei Patienten erlitten zusätzlich einen Korbhenkelriss des medialen Meniskus. Dieser wurde arthroskopisch bei dem einen Patient mit einer Naht versorgt, bei dem anderen mit zwei Ankern refixiert. Zwei Patienten hatten sich einen nicht näher bezeichneten Riss des medialen Meniskus, ein anderer des medialen und des lateralen Meniskus zugezogen, diese wurden genäht. Ein Außenmeniskushinterhornlängsriss wurde mittels Hinterhornteilresektion therapiert. Bei einem Patienten wurde eine Osteochondrosis dissecans diagnostiziert, die jedoch keiner Therapie bedurfte. Bei einem anderen wurde am betroffenen Knie eine Patellalateralisation mit einem arthroskopischen lateralen Patellarelease versorgt. Bei einem weiteren machte eine Synovitis eine partielle Synovektomie erforderlich. Bei 16 Patienten trat die VKB-Ruptur isoliert von anderen Verletzungen des Kniegelenkes auf.

\subsubsection{Behandlung und Operationsmethoden}

\subsubsection{Transplantate}

In 23 Fällen wurde als Bandersatz ein autologes Hamstringtransplantat verwendet. Zweimal wurde autologe Patellasehne transplantiert, wobei jeweils nur am proximalen Ende ein Knochenblock mit entnommen wurde. 


\subsubsection{Kanalanlage}

Der Knochenkanal für das Transplantat wurde bei 23 Patienten sowohl femoral als auch tibial durch die Wachstumsfuge gebohrt. Bei den zwei weiteren Patienten erfolgte die Kanalanlage beidseits fugenschonend, der Bandersatz wurde hier weder durch die femorale noch durch die tibiale Fuge gelegt.

Der femorale Kanal wurde bei 21 Patienten über das anteromediale Portal angelegt, bei zwei wurde der Kanal transtibial gebohrt, für zwei wurde keine Angabe gemacht.

Die Evaluation der Bohrkanalanlage erfolgte in 14 Fällen mit konventionellem Röntgen, bei einem Patienten mittels Magnetresonanztomographie (MRT) und bei neun Patienten ohne eine Bildgebung. Für einen wurde hierzu keine Angabe gemacht.

Für den tibialen Bohrkanal wurde ein Durchmesser von durchschnittlich 6,7 (4,5 bis 8) mm gewählt, femoral betrug dieser durchschnittlich 6,5 (4 bis 8) mm, wobei für den femoralen Durchmesser bei zwei Patienten keine Angabe gemacht wurde.

Zweimal wurde ein Handbohrer benutzt, 22-mal wurde elektrisch gebohrt, einmal wurde hierzu keine Angabe gemacht.

\subsubsection{Fixierung}

Im femoralen Knochenkanal wurde das Transplantat bei 17 Patienten mit einem Endobutton befestigt. Zweimal wurde es mit einer Interferenzschraube, die längs in den Knochenkanal eingeschraubt wurde, in diesem verkeilt. Einmal wurde es durch einen Stift fixiert, der senkrecht zum Kanalverlauf durch den Knochen und das Transplantat getrieben wurde. Fünfmal wurde das Transplantat fremdmaterialfrei mit einer Press-Fit-Methode im Knochenkanal befestigt, bei der das Transplantat mit körpereigenen Knochenzylindern verkeilt wird. 


\section{Femorale Fixierung}

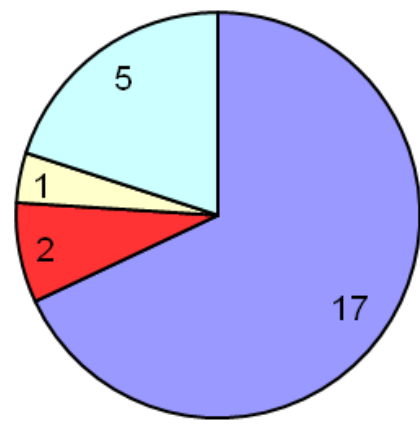

-Endobutton

口Interferenzschraube

口Transfixation

口Press-Fit

\section{Abbildung 4: Femorale Fixierung des Transplantats}

Tibial waren die Methoden vielfältiger. Fünfmal wurde das Transplantat mit einer Interferenzschraube verkeilt, sechsmal mit einem Suture Washer, also an einer Art Metallknopf, aufgehängt. Dreimal wurde es an einer Ankerschraube fixiert, zweimal mit einer Titankrampe gesichert und einmal mit einer Schraube und zusätzlicher Krampe fixiert. Einmal wurde es mit einem senkrecht durch den Kanal verlaufenden Stift (cross pin) und zweimal mit einer Pollerschraube befestigt. Einmal wurde es nur mit dem Periost vernäht, einmal an das Periost genäht und zusätzlich mit einer Pollerschraube gesichert, dreimal mit der oben beschriebenen Press-Fit-Methode befestigt.

\section{Tibiale Fixierung}

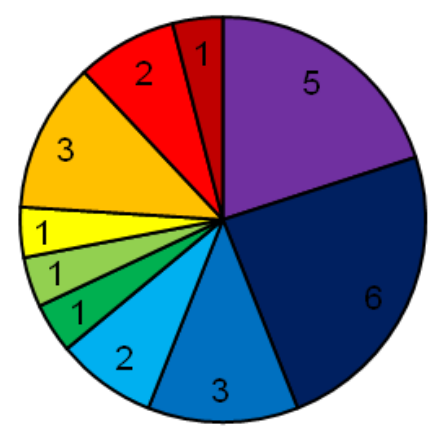

口Interferenzschraube
$\square$ Suture Washer
$\square$ Ankerschraube
$\square$ Titankrampe
$\square$ Cross Pin
$\square$ Naht an Periost
$\square$ Naht an Periost + Pollerschraube
$\square$ Press-Fit
$\square$ Pollerschraube
$\square$ Schraube + Krampe

Abbildung 5: Tibiale Fixierung des Transplantats 


\subsubsection{Nachbehandlung der Patienten}

Allen Patienten wurde postoperativ eine Sportpause von durchschnittlich 5,6 (1 bis 12) Monaten verordnet. Die durchschnittliche tatsächlich eingehaltene Sportpause betrug 7,1 (2 bis 16) Monate, wobei für acht Patienten keine Angabe über die tatsächliche Sportpause gemacht wurde. 21 Patienten durften das betroffene Bein nach der Operation nur teilbelasten. Dies galt für durchschnittlich 4,0 Wochen (5 Tage bis 6 Wochen), wobei für einen Patienten keine Aussage gemacht wurde, wie lange er das Bein nur teilweise belasten durfte. Vier Patienten durften direkt nach der Operation vollbelasten. Alle Patienten trugen nach der Operation eine Orthese, durchschnittlich für 8,0 (6 bis 12) Wochen.

20 Patienten wurde Krankengymnastik verordnet. Die durchschnittliche Dauer betrug 15,7 Wochen (6 Wochen bis 12 Monate), wobei für acht Patienten nicht angegeben war, wie lange die Krankengymnastik verordnet wurde. Zwei Patienten wurde keine Krankengymnastik verordnet. Für drei Patienten wurden keine Angaben darüber gemacht, ob Krankengymnastik verordnet wurde oder nicht.

\subsubsection{Ergebnisse der Nachuntersuchungen}

\subsubsection{Klinisches Ergebnis}

23 der 25 Patienten erreichten einen durchschnittlichen Lysholm-Score von 93,0 (42 bis 100) Punkten, bei zwei Patienten wurde kein Lysholm-Score erhoben. Bei der Berechnung des $I K D C$ erreichten sieben Patienten die Kategorie A, dreizehn B, einer C und einer D. Für drei Patienten wurde der IKDC nicht erhoben. Der Patient mit einem Lysolm-Score von 42 Punkten und dem als D klassifizierten IKDC hatte kurz vor der Nachuntersuchung eine erneute Distorsion mit Reruptur erlitten. 


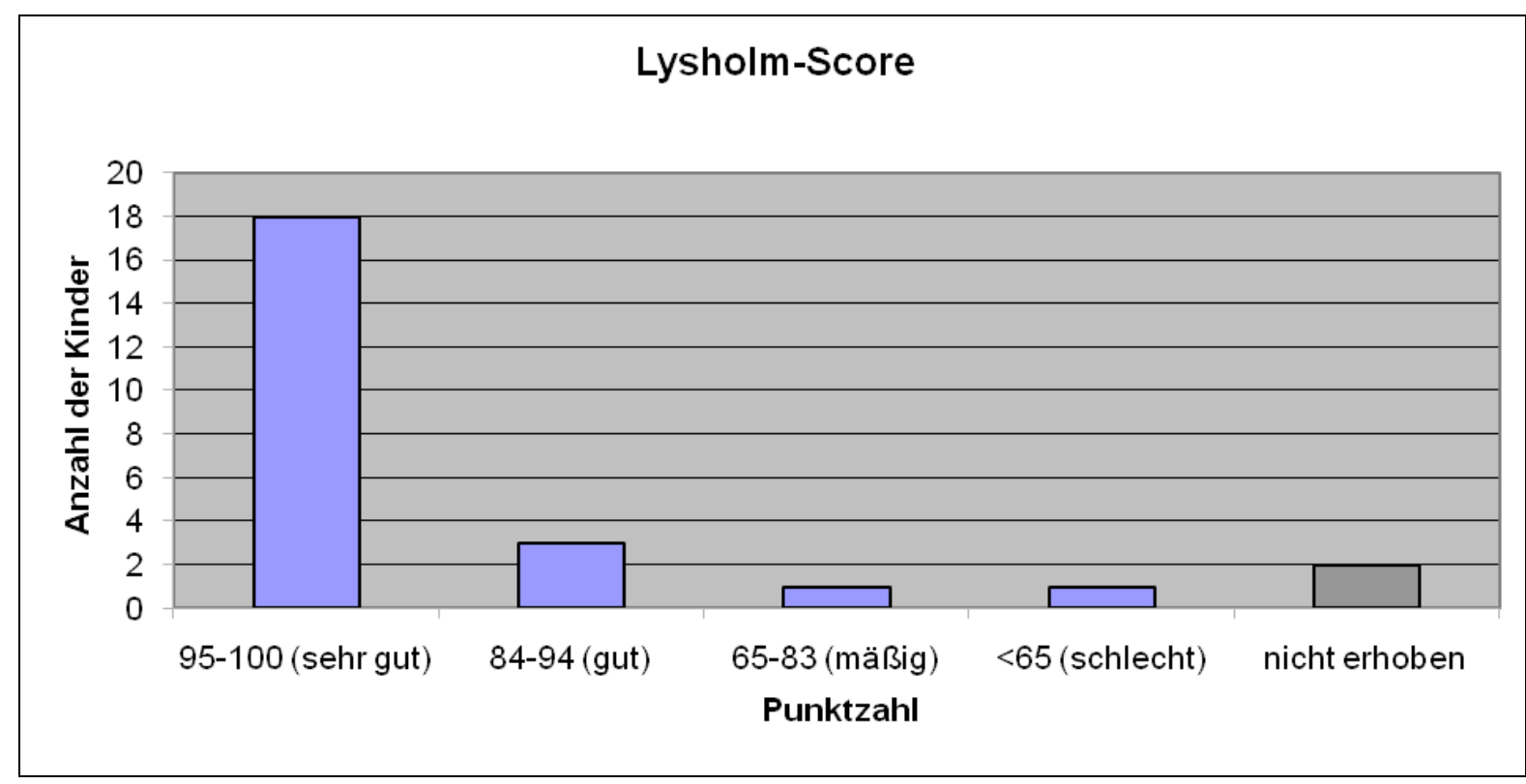

Abbildung 6: Lysholm-Score bei Nachuntersuchung

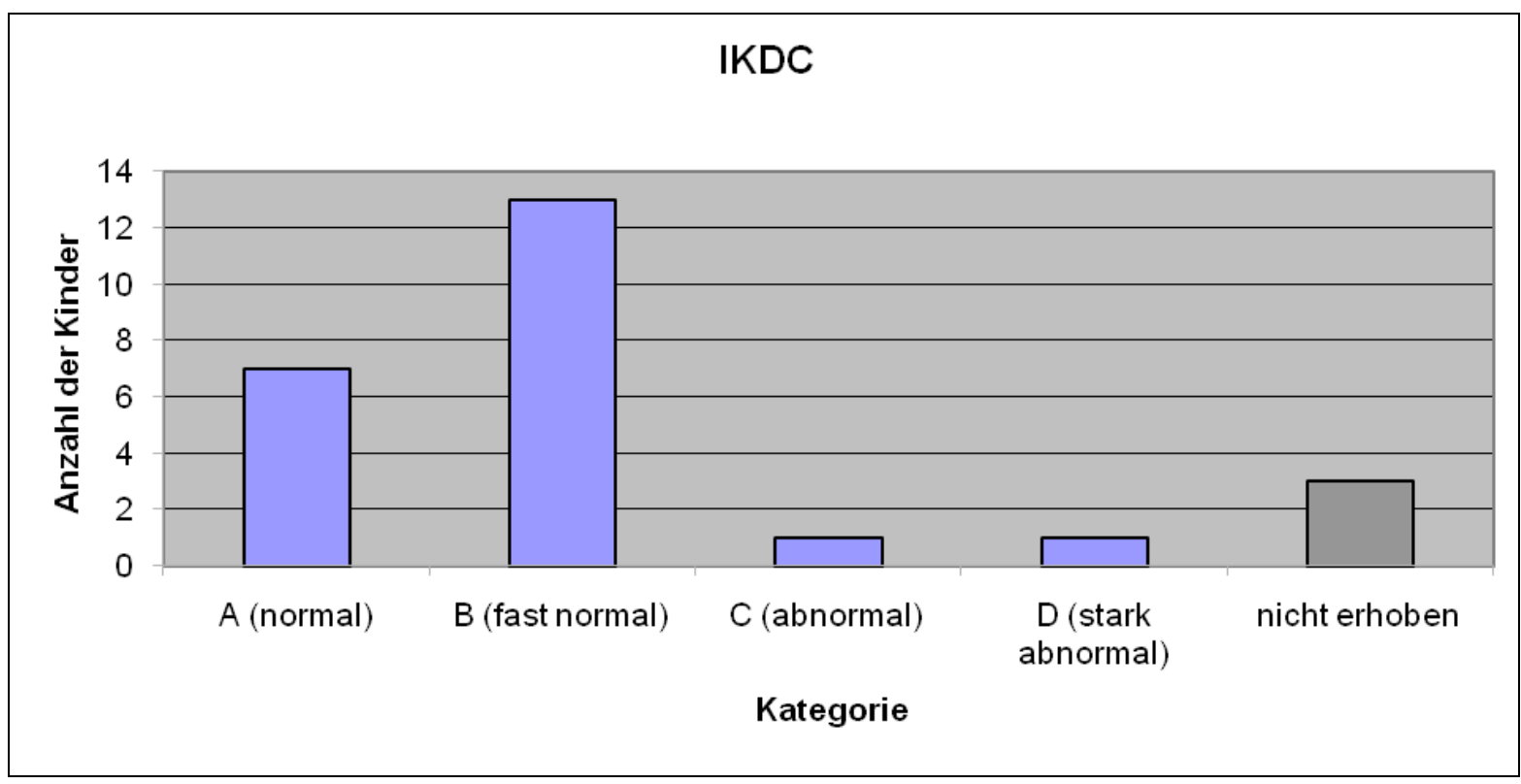

Abbildung 7: IKDC bei Nachuntersuchung

\subsubsection{Gemessene Bandlaxizität (KT-1000)}

Bei 19 Patienten wurde die Bandlaxizität des VKB instrumentell gemessen. Durchschnittlich ergab sich bei diesen Patienten bei Messung mit maximaler Zugkraft eine Seitendifferenz zwischen dem betroffenen und dem nicht betroffenen Bein von $+1,0(-2$ bis +5$) \mathrm{mm}$ auf der operierten Seite. Die operierte Seite lag bei durchschnittlich 8,6 (4 bis 15) mm, die Gegenseite bei durchschnittlich 7,7 (3 bis 12) mm. 


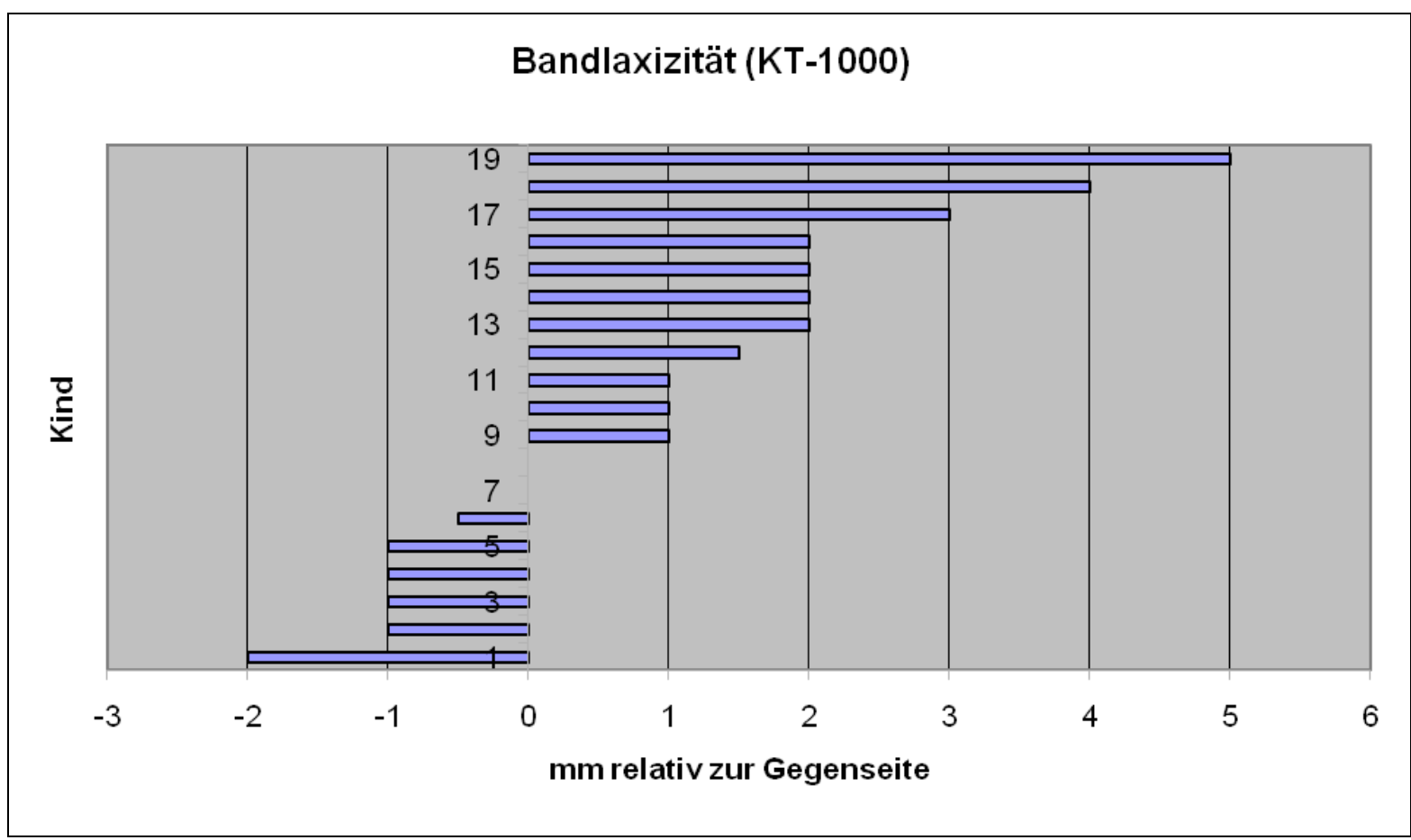

Abbildung 8: Gemessene Bandlaxizität (KT-1000) bei Nachuntersuchung relativ zur Gegenseite

\subsubsection{Postoperative Beinlängendifferenzen und Achsenabweichungen}

Sechs Patienten hatten eine Beinlängendifferenz oder Achsenabweichung entwickelt.

Ein Patient wies eine isolierte Achsenabweichung in Form eines Genu recurvatum von fünf Grad, ein anderer von zehn Grad im Vergleich zur Gegenseite auf. Zwei Patienten entwickelten eine relative Verkürzung des operierten Beines von $10 \mathrm{~mm}$. Bei einem anderen vergrößerte sich eine bereits präoperativ existierende Beinlängendifferenz postoperativ um minus $10 \mathrm{~mm}$ auf der der operierten Seite, zusätzlich wurde bei diesem Patienten ein Genu recurvatum von zehn Grad im Vergleich zur Gegenseite gemessen. Ein Patient hatte bei gleichzeitiger relativer Verkürzung von $30 \mathrm{~mm}$ und zehn Grad Achsenabweichung in Form eines Genu recurvatum eine Valgusabweichung von acht Grad entwickelt. Die von dem Nachuntersucher vermutete Ursache für das Entstehen der Wachstumsstörung bei diesem Patienten war, dass der femorale Kanal zu weit dorsal gebohrt wurde und den Ranvierschen Schnürring verletzte.

Von anderen klinischen Fehlstellungen des betroffenen Knies wurde nicht berichtet. Für die Feststellung einer Wachstumsstörung konnte bei vier Patienten auf ein aktuelles Röntgenbild und bei zwei weiteren auf eine aktuelle MRT-Untersuchung zurückgegriffen werden. Für einen Patienten wurden sowohl ein Röntgenbild als auch ein MRT angefertigt. Bei den 
anderen Patienten wurden diese Daten rein klinisch erhoben oder es wurde keine Angabe zu MRT oder konventionellen Röntgenuntersuchungen gemacht.

\section{Fehlwachstum}

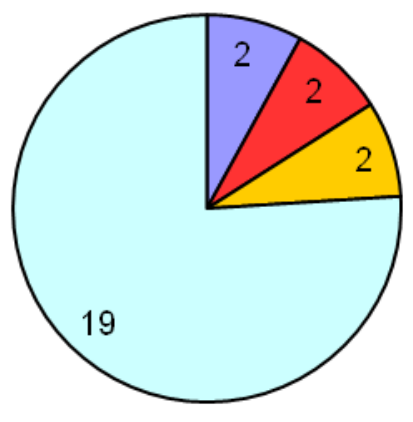

口Isolierte Beinlängendifferenz

口Isolierte Achsenabweichung

口Beinlängendifferenz+

Achsenabweichung

口Kein Fehlwachstum

Abbildung 9: Fehlwachstum im Vergleich zur Gegenseite zum Zeitpunkt der Nachuntersuchung

\subsubsection{Rerupturen, degenerative Veränderungen und weitere Komplikationen}

Bei einem der 25 Patienten kam es innerhalb des Zeitraums bis zur Nachuntersuchung zu einer erneuten Ruptur des VKB. Bei zwei Patienten wurde bei der Nachuntersuchung jeweils eine Crepitatio retropatellaris festgestellt. In bildgebenden Verfahren wurden jedoch bei keinem Kind degenerative Veränderungen detektiert. Das betroffene Knie eines weiteren der 25 Patienten musste erneut operiert werden, da er sich in der Zwischenzeit eine Verletzung des Innenmeniskus zugezogen hatte. 


\subsubsection{Operationsergebnisse in Abhängigkeit unterschiedlicher Methoden}

Tabelle 2: Operationsergebnisse nach unterschiedlichen Methoden (1)

\begin{tabular}{|l|l|l|l|}
\hline & Alle Patienten & $\begin{array}{l}\text { Wachstumsfugenschonende } \\
\text { Operationstechnik }\end{array}$ & $\begin{array}{l}\text { Wachstumsfugenkreuzende } \\
\text { Operationstechnik }\end{array}$ \\
\hline Anzahl der Patienten & 25 & 2 & 23 \\
\hline Durchschnittsalter (Jahre) & $10,4(7-12)$ & $12,0(12-12)$ & $10,3(7-12)$ \\
\hline Lysholm-Score & $\begin{array}{l}93,0(42 \text { bis } 100) \\
2 \times \mathrm{k} . \mathrm{A} .\end{array}$ & k.A. & $93,0(42-100)$ \\
\hline IKDC & $\begin{array}{l}7 \times \mathrm{A}, 13 \times \mathrm{B} \\
1 \times \mathrm{C}, 1 \times \mathrm{D}, 3 \mathrm{x} \\
\mathrm{k} . \mathrm{A} .\end{array}$ & $1 \times \mathrm{C}, 1 \times \mathrm{B}$ & $\begin{array}{l}7 \times \mathrm{A}, 12 \times \mathrm{B}, 1 \times \mathrm{D}, 3 \times \\
\mathrm{k} . \mathrm{A} .\end{array}$ \\
\hline $\begin{array}{l}\text { Rerupturrate } \\
\text { Beinlängendifferenzen oder }\end{array}$ & 6 von $25(24 \%)$ & 2 von 2(100\%) & 1 von $23(4,3 \%)$ \\
\hline
\end{tabular}

Tabelle 3: Operationsergebnisse nach unterschiedlichen Methoden (2)

\begin{tabular}{|c|c|c|c|c|}
\hline & $\begin{array}{l}\text { Autologes BTB- } \\
\text { Transplantat }\end{array}$ & $\begin{array}{l}\text { Autologes } \\
\text { Hamstring- } \\
\text { transplantat }\end{array}$ & $\begin{array}{l}\text { Zugang über } \\
\text { Anteromediales } \\
\text { Portal }\end{array}$ & $\begin{array}{l}\text { Zugang } \\
\text { transtibial }\end{array}$ \\
\hline Anzahl der Patienten & 2 & 23 & 21 & 2 \\
\hline Durchschnittsalter (Jahre) & $12,0(12-12)$ & $10,3(7-12)$ & $10,3(7-12)$ & $10,5(9-12)$ \\
\hline Lysholm-Score & k.A. & $93,0(42-100)$ & $\begin{array}{l}92,3(42-100,2 \text { x } \\
\text { k.A.) }\end{array}$ & $95(90-100)$ \\
\hline$I K D C$ & $1 \times \mathrm{C}, 1 \times \mathrm{B}$ & $\begin{array}{l}7 \times \mathrm{A}, 12 \times \mathrm{B}, 1 \times \\
\mathrm{D}, 3 \times \mathrm{k} . \mathrm{A} .\end{array}$ & $\begin{array}{l}6 \times \mathrm{A}, 10 \times \mathrm{B}, 1 \times \\
\mathrm{C}, 1 \times \mathrm{D} 3 \times \mathrm{k} . \mathrm{A} .\end{array}$ & $1 \times \mathrm{A}, 1 \times \mathrm{B}$ \\
\hline Rerupturrate & 0 von $2(0 \%)$ & 1 von $23(4,3 \%)$ & 1 von $21(4,8 \%)$ & 0 von $2(0 \%)$ \\
\hline $\begin{array}{l}\text { Beinlängendifferenzen oder } \\
\text { Achsabweichungen }\end{array}$ & 2 von $2(100 \%)$ & 4 von $23(17,4 \%)$ & 5 von $21(23,8 \%)$ & 0 von $2(0 \%)$ \\
\hline
\end{tabular}




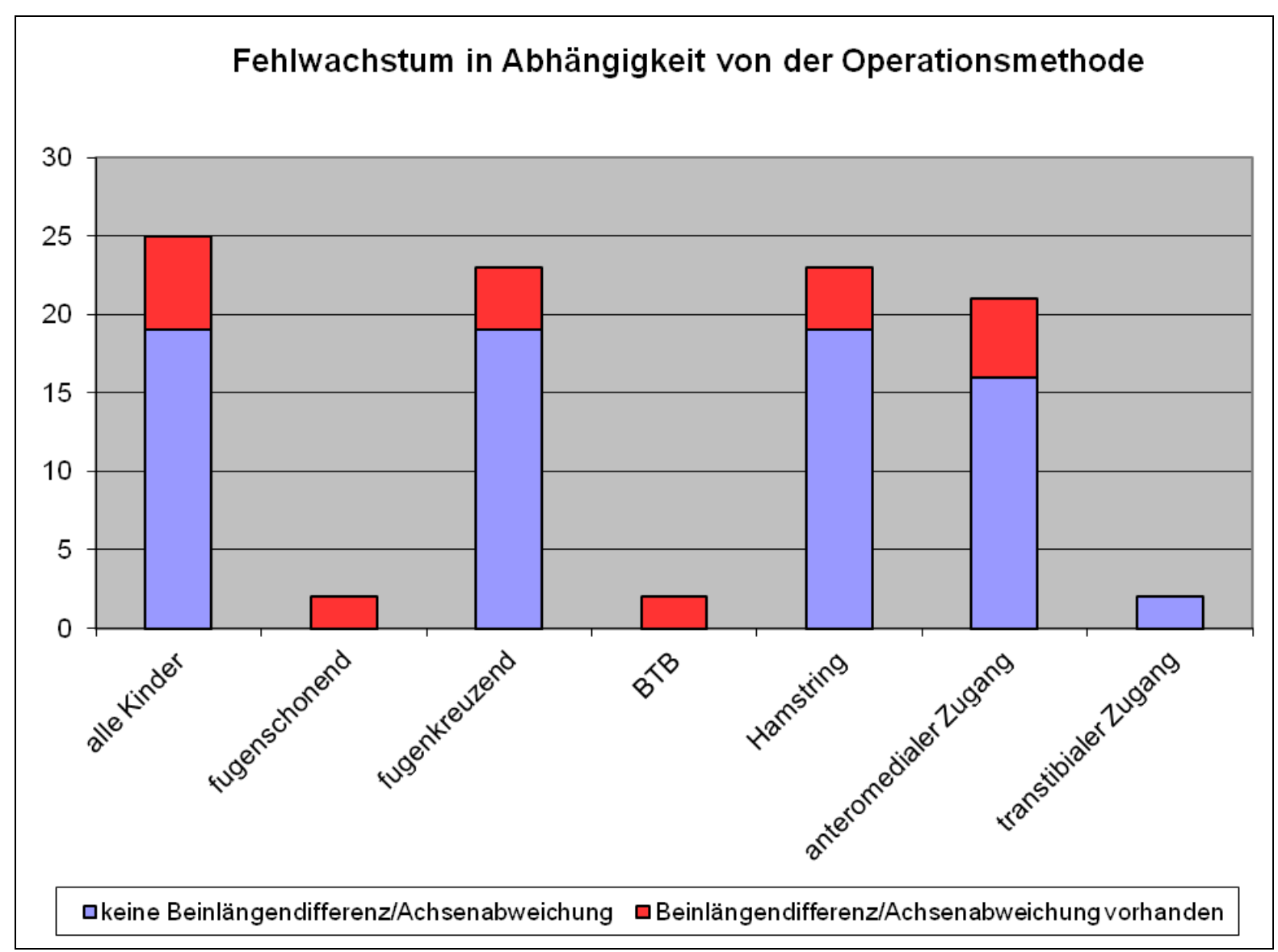

Abbildung 10: Entwicklung einer postoperativen Beinlängendifferenz oder Achsenabweichung in Abhängigkeit von der Operationsmethode

\subsection{Systematische Literaturanalyse}

\subsubsection{Ergebnisse der Literatursuche}

Zum Zeitpunkt der letzten Aktualisierung der Suchanfrage am 17. Juli 2008 ergab sich mit der oben genannten Suchformel verteilt auf alle Datenbanken eine Gesamtzahl von 1811 Treffern. Diese verteilten sich auf 947 Treffer in der Literaturdatenbank Medline, 58 in der Cochrane Library, 48 bei SpringerLink, sieben im Thieme Verlag und 751 Treffern in der Literaturdatenbank Embase (Excerpta Medica Database).

Nach Studium der Publikationstitel, sowie falls nötig und möglich der Zusammenfassungen, wurden insgesamt 120 Artikel beschafft und gelesen.

Endgültig wurden 59 Artikel ausgewertet, auf die die Ein- und Ausschlusskriterien zutrafen. 


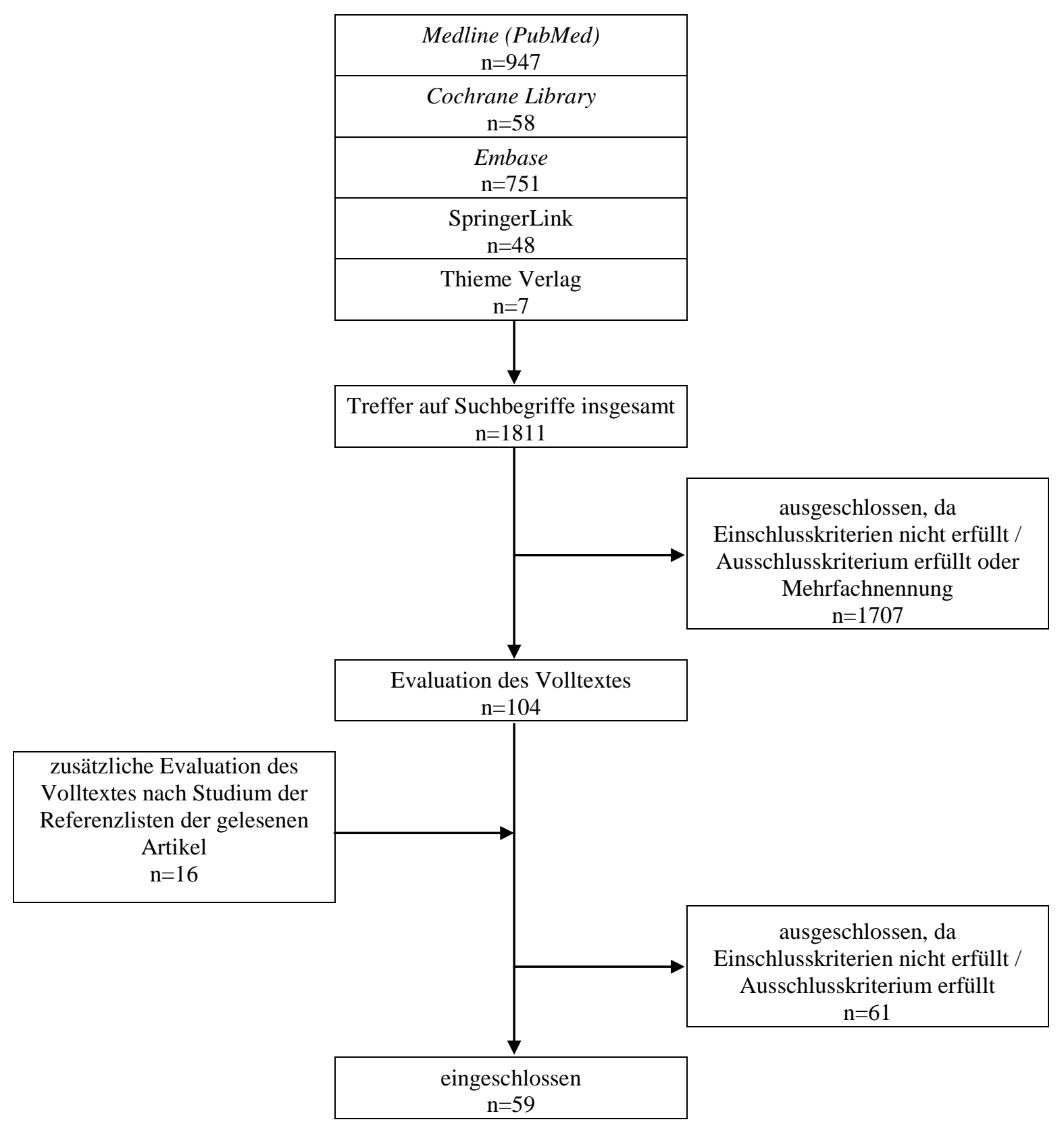

Abbildung 11: Ablauf der Studienselektion

Bei den allermeisten Publikationen handelte es sich um Fallserien (Level of Evidence IV), nur wenige Fall-Kontroll-Studien (Level of Evidence IIIb) wurden identifiziert (Aichroth et al. 2002; Arbes et al. 2007; Janarv et al. 1996; McCarroll et al. 1988; McCarroll et al. 1994; Moksnes et al. 2008; Pressman et al. 1997). Randomisiert-kontrollierte Studien (Level of Evidence $I b$ ) oder Kohortenstudien (Level of Evidence (IIb) konnten nicht gefunden werden. 


\subsubsection{Ergebnisse der Literaturanalyse}

\section{Tabelle 4: Kreuzbandplastik: Therapieergebnis im Wachstumsalter (bis maximal zwölf Jahre bei OP)}

\begin{tabular}{|c|c|}
\hline $\begin{array}{l}\text { Anzahl der Artikel, die in die } \\
\text { Auswertung eingebunden } \\
\text { werden konnten }\end{array}$ & $\begin{array}{l}13 \\
\text { (Aronowitz et al. 2000; Edwards und Grana 2001; Guzzanti et al. 2003b; Knorr et } \\
\text { al. 2006; Lipscomb und Anderson 1986; Lo et al. 1997; Lukas et al. 2007; Matava } \\
\text { und Siegel 1997; Micheli et al. 1999; Moksnes et al. 2008; Nakhostine et al. 1995; } \\
\text { Robert und Bonnard 1999; Sankar et al. 2006) }\end{array}$ \\
\hline Anzahl der Patienten & 37 \\
\hline Durchschnittsalter (Jahre) & $\begin{array}{l}\text { 10,7 (2-12) } \\
\text { (Aronowitz et al. 2000; Edwards und Grana 2001; Guzzanti et al. 2003b; Knorr et } \\
\text { al. 2006; Lipscomb und Anderson 1986; Lo et al. 1997; Lukas et al. 2007; Matava } \\
\text { und Siegel 1997; Micheli et al. 1999; Moksnes et al. 2008; Nakhostine et al. 1995; } \\
\text { Robert und Bonnard 1999; Sankar et al. 2006) }\end{array}$ \\
\hline $\begin{array}{l}\text { Klinisches Ergebnis } \\
\text { (Lysholm-Score) }\end{array}$ & $\begin{array}{l}\text { 95,6 (84 - 100) } \\
\text { (Aronowitz et al. 2000; Edwards und Grana 2001; Knorr et al. 2006; Lukas et al. } \\
\text { 2007; Matava und Siegel 1997; Micheli et al. 1999; Moksnes et al. 2008; Sankar et } \\
\text { al. 2006) }\end{array}$ \\
\hline Rerupturrate & 0 von $37(0 \%)$ \\
\hline $\begin{array}{l}\text { Beinlängendifferenzen oder } \\
\text { Achsenabweichungen }\end{array}$ & $\begin{array}{l}2 \text { von } 32(6,3 \%) \\
(1 \text { x Beinlängendifferenz, } 1 \text { x Genu recurvatum) } \\
\text { (Micheli et al. 1999; Nakhostine et al. 1995) }\end{array}$ \\
\hline
\end{tabular}

Tabelle 5: Kreuzbandplastik im Wachstumsalter (bis maximal zwölf Jahre bei OP): Wachstumsfugenschonende Operationstechniken versus transphysäre Techniken

\begin{tabular}{|l|l|l|}
\hline & $\begin{array}{l}\text { Wachstumsfugenschonende } \\
\text { Operationstechnik }\end{array}$ & $\begin{array}{l}\text { Wachstumsfugenkreuzende } \\
\text { Operationstechnik }\end{array}$ \\
\hline $\begin{array}{l}\text { Anzahl der Artikel, die in die } \\
\text { Auswertung eingebunden } \\
\text { werden konnten }\end{array}$ & $\begin{array}{l}5 \\
\text { (Guzzanti et al. 2003b; Knorr et al. 2006; } \\
\text { Micheli et al. 1999; Nakhostine et al. } \\
1995 ; \text { Robert und Bonnard 1999) }\end{array}$ & $\begin{array}{l}\text { (Aronowitz et al. 2000; Edwards und } \\
\text { Grana 2001; Lukas et al. 2007; Matava } \\
\text { und Siegel 1997; Sankar et al. 2006) }\end{array}$ \\
\hline Anzahl der Patienten & 18 & $\begin{array}{l}12 \\
\text { Durchschnittsalter (Jahre) }\end{array}$ \\
$\begin{array}{l}10,2 \text { (2-12) } \\
\text { (Guzzanti et al. 2003b; Knorr et al. 2006; } \\
\text { Micheli et al. 1999; Nakhostine et al. } \\
1995 ; \text { Robert und Bonnard 1999) }\end{array}$ & $\begin{array}{l}\text { (Aronowitz et al. 2000; Edwards und } \\
\text { und Siegel 1997; Sankar et al. 2006) }\end{array}$ \\
\hline
\end{tabular}




\begin{tabular}{|l|l|l|}
\hline $\begin{array}{l}\text { Klinisches Ergebnis } \\
\text { (Lysholm-Score })\end{array}$ & $\begin{array}{l}96,8(93-100) \\
\text { (Knorr et al. 2006; Micheli et al. 1999) }\end{array}$ & $\begin{array}{l}96(89-100) \\
\text { (Aronowitz et al. 2000; Edwards und } \\
\text { Grana 2001; Lukas et al. 2007; Matava } \\
\text { und Siegel 1997; Sankar et al. 2006) }\end{array}$ \\
\hline Rerupturrate & 0 von 18 (0\%) & 0 von 12 (0\%) \\
\hline $\begin{array}{l}\text { Beinlängendifferenzen oder } \\
\text { Achsenabweichungen }\end{array}$ & $\begin{array}{l}\text { 2 von 18 (11,1\%) } \\
(1 \times \text { Beinlängendifferenz, 1 x Genu } \\
\text { recurvatum) } \\
\text { (Micheli et al. 1999; Nakhostine et al. } \\
1995)\end{array}$ & 0 von 12 (0\%) \\
\hline
\end{tabular}

Tabelle 6: Kreuzbandplastik bei Patienten im Wachstumsalter (altersunabhängig): wachstumsfugenschonende versus wachstumsfugenkreuzende Techniken

\begin{tabular}{|c|c|c|}
\hline & $\begin{array}{l}\text { Wachstumsfugenschonende } \\
\text { Operationstechnik }\end{array}$ & Wachstumsfugenkreuzende Operationstechnik \\
\hline $\begin{array}{l}\text { Anzahl der Artikel, } \\
\text { die in die Auswertung } \\
\text { eingebunden werden } \\
\text { konnten }\end{array}$ & $\begin{array}{l}15 \\
\text { (Anderson 2003; Benedetto et al. } \\
\text { 1996; Brief 1991; Gebhard et al. } \\
\text { 2006; Guzzanti et al. 2003b; } \\
\text { Janarv et al. 1996; Knorr et al. } \\
\text { 2006; Kocher et al. 2005; Letts et } \\
\text { al. 2000; McCarroll et al. 1988; } \\
\text { Micheli et al. 1999; Nakhostine et } \\
\text { al. 1995; Parker et al. 1994; } \\
\text { Robert und Bonnard 1999; Seil } \\
\text { und Robert 2005) }\end{array}$ & $\begin{array}{l}27 \\
\text { (Aichroth et al. 2002; Arbes et al. 2007; Aronowitz et } \\
\text { al. 2000; Attmanspacher et al. 2002; Attmanspacher } \\
\text { et al. 2003; Bollen et al. 2008; Casper et al. 2006; } \\
\text { Edwards und Grana 2001; Fankhauser et al. 2004; } \\
\text { Fuchs et al. 2002; Gaulrapp und Haus 2003; } \\
\text { Gaulrapp und Haus 2006; Gebhard et al. 2006; Gorin } \\
\text { et al. 2003; Kocher et al. 2007; Koman und Sanders } \\
\text { 1999; Lukas et al. 2007; Matava und Siegel 1997; } \\
\text { McCarroll et al. 1994; McIntosh et al. 2006; } \\
\text { Pressman et al. 1997; Sankar et al. 2006; Seon et al. } \\
\text { 2005; Shelbourne et al. 2004; Sobau und Ellermann } \\
\text { 2004; Thompson et al. 2006; Vaquero et al. 2005) }\end{array}$ \\
\hline Anzahl der Patienten & 154 & 489 \\
\hline $\begin{array}{l}\text { Durchschnittsalter } \\
\text { (Jahre) }\end{array}$ & $\begin{array}{l}\text { 11,9 (2-17) } \\
\text { (Anderson 2003; Brief 1991; } \\
\text { Guzzanti et al. 2003b; Janarv et al. } \\
\text { 1996; Knorr et al. 2006; Kocher et } \\
\text { al. 2005; Letts et al. 2000; Micheli } \\
\text { et al. 1999; Nakhostine et al. } \\
\text { 1995; Parker et al. 1994; Robert } \\
\text { und Bonnard 1999) }\end{array}$ & $\begin{array}{l}\text { 13,8 (9-17) } \\
\text { (Aichroth et al. 2002; Aronowitz et al. 2000; } \\
\text { Attmanspacher et al. 2002; Bollen et al. 2008; Casper } \\
\text { et al. 2006; Edwards und Grana 2001; Fankhauser et } \\
\text { al. 2004; Fuchs et al. 2002; Gorin et al. 2003; Kocher } \\
\text { et al. 2007; Koman und Sanders 1999; Lukas et al. } \\
\text { 2007; Matava und Siegel 1997; McCarroll et al. } \\
\text { 1994; McIntosh et al. 2006; Sankar et al. 2006; Seon } \\
\text { et al. 2005; Shelbourne et al. 2004; Vaquero et al. } \\
\text { 2005) }\end{array}$ \\
\hline $\begin{array}{l}\text { Klinisches Ergebnis } \\
\text { (Lysholm-Score) }\end{array}$ & $\begin{array}{l}\text { 95,3 (74 - 100) } \\
\text { (Janarv et al. 1996; Knorr et al. } \\
\text { 2006; Kocher et al. 2005; Micheli } \\
\text { et al. 1999; Parker et al. 1994) }\end{array}$ & $\begin{array}{l}\text { 94,4 (89-100) } \\
\text { (Aronowitz et al. 2000; Attmanspacher et al. 2002; } \\
\text { Edwards und Grana 2001; Fuchs et al. 2002; } \\
\text { Gaulrapp und Haus 2003; Gaulrapp und Haus 2006; }\end{array}$ \\
\hline
\end{tabular}




\begin{tabular}{|c|c|c|}
\hline & & $\begin{array}{l}\text { Kocher et al. 2007; Lukas et al. 2007; McIntosh et al. } \\
\text { 2006; Sankar et al. 2006; Seon et al. 2005) }\end{array}$ \\
\hline Rerupturrate & $\begin{array}{l}3 \text { von } 154(1,9 \%) \\
\text { (Kocher et al. 2005; McCarroll et } \\
\text { al. 1988) }\end{array}$ & $\begin{array}{l}24 \text { von } 489(4,9 \%) \\
\text { (Aichroth et al. 2002; Edwards und Grana 2001; } \\
\text { Fankhauser et al. 2004; Gaulrapp und Haus 2006; } \\
\text { Gebhard et al. 2006; Kocher et al. 2007; Matava und } \\
\text { Siegel 1997; McCarroll et al. 1994; McIntosh et al. } \\
\text { 2006; Shelbourne et al. 2004; Sobau und Ellermann } \\
\text { 2004; Thompson et al. 2006; Vaquero et al. 2005) }\end{array}$ \\
\hline $\begin{array}{l}\text { Beinlängendifferenzen } \\
\text { oder } \\
\text { Achsenabweichungen }\end{array}$ & $\begin{array}{l}17 \text { von } 154(11,0 \%) \\
(7 \text { x Beinlängendifferenz, } 2 \text { x } \\
\text { Genu valgum, } 1 \text { x Genu varum, } 7 \\
\text { x Genu recurvatum) } \\
\text { (Anderson 2003; Micheli et al. } \\
\text { 1999; Nakhostine et al. 1995; } \\
\text { Parker et al. 1994; Seil und Robert } \\
\text { 2005) }\end{array}$ & $\begin{array}{l}7 \text { von } 489(1,4 \%) \\
(6 \text { x Beinlängendifferenz, } 1 \text { x Genu valgum) } \\
\text { (Edwards und Grana 2001; Koman und Sanders } \\
\text { 1999; McIntosh et al. 2006; Seon et al. 2005) }\end{array}$ \\
\hline
\end{tabular}

Tabelle 7: Kreuzbandplastik im Wachstumsalter (bis maximal zwölf Jahre bei OP): Ergebnisse in Abhängigkeit vom verwendeten Transplantat

\begin{tabular}{|c|c|c|c|c|c|}
\hline & Patellasehne & Hamstring & Quadrizepssehne & $\begin{array}{l}\text { Fascia } \\
\text { lata/Iliotibialband }\end{array}$ & Allograft \\
\hline $\begin{array}{l}\text { Anzahl der } \\
\text { Artikel, die in } \\
\text { die Auswertung } \\
\text { eingebunden } \\
\text { werden konnten }\end{array}$ & $\begin{array}{l}3 \\
\text { (Knorr et al. } \\
\text { 2006; Lukas } \\
\text { et al. 2007; } \\
\text { Robert und } \\
\text { Bonnard } \\
\text { 1999) }\end{array}$ & $\begin{array}{l}6 \\
\text { (Edwards und } \\
\text { Grana 2001; } \\
\text { Guzzanti et al. } \\
\text { 2003b; Lipscomb } \\
\text { und Anderson } \\
\text { 1986; Lo et al. } \\
\text { 1997; Matava und } \\
\text { Siegel 1997; } \\
\text { Moksnes et al. } \\
\text { 2008) }\end{array}$ & 0 & $\begin{array}{l}2 \\
\text { (Micheli et al. } \\
\text { 1999; Nakhostine } \\
\text { et al. 1995) }\end{array}$ & $\begin{array}{l}2 \\
\text { (Aronowitz et } \\
\text { al. 2000; } \\
\text { Sankar et al. } \\
\text { 2006) }\end{array}$ \\
\hline $\begin{array}{l}\text { Anzahl der } \\
\text { Patienten }\end{array}$ & 12 & 14 & 0 & 6 & 5 \\
\hline $\begin{array}{l}\text { Durchschnitts- } \\
\text { alter (Jahre) }\end{array}$ & $\begin{array}{l}\text { 10,3 (5-12) } \\
\text { (Knorr et al. } \\
\text { 2006; Lukas } \\
\text { et al. 2007; } \\
\text { Robert und } \\
\text { Bonnard } \\
\text { 1999) }\end{array}$ & $\begin{array}{l}11(8-12) \\
\text { (Edwards und } \\
\text { Grana 2001; } \\
\text { Guzzanti et al. } \\
\text { 2003b; Lipscomb } \\
\text { und Anderson } \\
\text { 1986; Lo et al. } \\
\text { 1997; Matava und } \\
\text { Siegel 1997; } \\
\text { Moksnes et al. }\end{array}$ & & $\begin{array}{l}9,8(2-12) \\
\text { (Micheli et al. } \\
\text { 1999; Nakhostine } \\
\text { et al. 1995) }\end{array}$ & $\begin{array}{l}11,6(11-12) \\
\text { (Aronowitz et } \\
\text { al. 2000; } \\
\text { Sankar et al. } \\
\text { 2006) }\end{array}$ \\
\hline
\end{tabular}




\begin{tabular}{|c|c|c|c|c|}
\hline & & 2008) & & \\
\hline $\begin{array}{l}\text { Klinisches } \\
\text { Ergebnis } \\
\text { (Lysholm- } \\
\text { Score) }\end{array}$ & $\begin{array}{l}96,5(90- \\
100) \\
\text { (Knorr et al. } \\
\text { 2006; Lukas } \\
\text { et al. 2007) }\end{array}$ & $\begin{array}{l}89,3(84-94) \\
\text { (Edwards und } \\
\text { Grana 2001; } \\
\text { Matava und Siegel } \\
\text { 1997; Moksnes et } \\
\text { al. 2008) }\end{array}$ & $\begin{array}{l}\text { 96,4 (93 - 100) } \\
\text { (Micheli et al. } \\
\text { 1999) }\end{array}$ & $\begin{array}{l}97,6(91- \\
100) \\
\text { (Aronowitz et } \\
\text { al. 2000; } \\
\text { Sankar et al. } \\
\text { 2006) }\end{array}$ \\
\hline Rerupturrate & 0 von $12(0 \%)$ & 0 von $14(0 \%)$ & 0 von $6(0 \%)$ & 0 von $5(0 \%)$ \\
\hline $\begin{array}{l}\text { Beinlängen- } \\
\text { differenzen } \\
\text { oder Achsen- } \\
\text { abweichungen }\end{array}$ & 0 von $12(0 \%)$ & 0 von $9(0 \%)$ & $\begin{array}{l}2 \text { von } 6(33,3 \%) \\
\text { (1 x Beinlängen- } \\
\text { differenz, } 1 \text { x } \\
\text { Genu recurvatum) } \\
\text { (Micheli et al. } \\
\text { 1999; Nakhostine } \\
\text { et al. 1995) }\end{array}$ & 0 von $5(0 \%)$ \\
\hline
\end{tabular}

Tabelle 8: Kreuzbandplastik im Wachstumsalter (altersunabhängig): Ergebnisse in Abhängigkeit vom verwendeten Transplantat

\begin{tabular}{|c|c|c|c|c|c|}
\hline & Patellasehne & Hamstring & Quadrizepssehne & $\begin{array}{l}\text { Fascia lata/ } \\
\text { Iliotibialband }\end{array}$ & Allograft \\
\hline $\begin{array}{l}\text { Anzahl der } \\
\text { Artikel, die in } \\
\text { die } \\
\text { Auswertung } \\
\text { eingebunden } \\
\text { werden } \\
\text { konnten }\end{array}$ & $\begin{array}{l}16 \\
\text { (Arbes et al. } \\
\text { 2007; Arndt et } \\
\text { al. 1999; } \\
\text { Benedetto et al. } \\
\text { 1996; Edwards } \\
\text { und Grana } \\
\text { 2001; Gaulrapp } \\
\text { und Haus 2003; } \\
\text { Janarv et al. } \\
\text { 1996; Knorr et } \\
\text { al. 2006; Lukas } \\
\text { et al. 2007; } \\
\text { McCarroll et al. } \\
\text { 1994; Millett et } \\
\text { al. 2002; } \\
\text { Moksnes et al. } \\
\text { 2008; Reilly } \\
\text { und Choit 2007; } \\
\text { Robert und } \\
\text { Bonnard 1999; } \\
\text { Seil und Robert } \\
\text { 2005; } \\
\text { Shelbourne et } \\
\text { al. 2004; } \\
\text { Wahler et al. }\end{array}$ & $\begin{array}{l}28 \\
\text { (Aichroth et al. 2002; } \\
\text { Anderson 2003; } \\
\text { Attmanspacher et al. } \\
\text { 2002; Attmanspacher } \\
\text { et al. 2003; Bisson et } \\
\text { al. 1998; Bollen et al. } \\
\text { 2008; Brief 1991; } \\
\text { Casper et al. 2006; } \\
\text { Edwards und Grana } \\
\text { 2001; Fankhauser et al. } \\
\text { 2004; Gaulrapp und } \\
\text { Haus 2006; Gebhard et } \\
\text { al. 2006; Guzzanti et } \\
\text { al. 2003b; Guzzanti et } \\
\text { al. 2003a; Hoffmann et } \\
\text { al. 1998; Janarv et al. } \\
\text { 1996; Kocher et al. } \\
\text { 2007; Koman und } \\
\text { Sanders 1999; Letts et } \\
\text { al. 2000; Lipscomb } \\
\text { und Anderson 1986; } \\
\text { Lo et al. 1997; Matava } \\
\text { und Siegel 1997; } \\
\text { McIntosh et al. 2006; } \\
\text { Millett et al. 2002; }\end{array}$ & $\begin{array}{l}2 \\
\text { (Gebhard et al. } \\
\text { 2006; Lo et al. } \\
\text { 1997) }\end{array}$ & $\begin{array}{l}5 \\
\text { (Gebhard et } \\
\text { al. 2006; } \\
\text { Kocher et al. } \\
\text { 2005; } \\
\text { McCarroll et } \\
\text { al. 1988; } \\
\text { Micheli et al. } \\
\text { 1999; } \\
\text { Nakhostine et } \\
\text { al. 1995) }\end{array}$ & $\begin{array}{l}7 \\
\text { (Andrews et } \\
\text { al. 1994; } \\
\text { Aronowitz et } \\
\text { al. 2000; } \\
\text { Fuchs et al. } \\
\text { 2002; Gorin et } \\
\text { al. 2003; } \\
\text { Millett et al. } \\
\text { 2002; Sankar } \\
\text { et al. 2006; } \\
\text { Vaquero et al. } \\
\text { 2005) }\end{array}$ \\
\hline
\end{tabular}




\begin{tabular}{|c|c|c|c|c|c|}
\hline & 1998) & $\begin{array}{l}\text { Moksnes et al. 2008; } \\
\text { Parker et al. 1994; } \\
\text { Seon et al. 2005; Sobau } \\
\text { und Ellermann 2004) }\end{array}$ & & & \\
\hline $\begin{array}{l}\text { Anzahl der } \\
\text { Patienten }\end{array}$ & 200 & 359 & 14 & 79 & 76 \\
\hline $\begin{array}{l}\text { Durchschnitts } \\
\text { alter (Jahre) }\end{array}$ & $\begin{array}{l}\text { 14,1 (5-17) } \\
\text { (Edwards und } \\
\text { Grana 2001; } \\
\text { Knorr et al. } \\
\text { 2006; Lukas et } \\
\text { al. 2007; } \\
\text { McCarroll et al. } \\
\text { 1994; Reilly } \\
\text { und Choit 2007; } \\
\text { Robert und } \\
\text { Bonnard 1999; } \\
\text { Shelbourne et } \\
\text { al. 2004; } \\
\text { Wahler et al. } \\
\text { 1998) }\end{array}$ & $\begin{array}{l}\text { 13,8 (8-17) } \\
\text { (Aichroth et al. 2002; } \\
\text { Anderson 2003; } \\
\text { Attmanspacher et al. } \\
\text { 2002; Attmanspacher } \\
\text { et al. 2003; Bollen et } \\
\text { al. 2008; Brief 1991; } \\
\text { Casper et al. 2006; } \\
\text { Edwards und Grana } \\
\text { 2001; Fankhauser et al. } \\
\text { 2004; Guzzanti et al. } \\
\text { 2003b; Guzzanti et al. } \\
\text { 2003a; Kocher et al. } \\
\text { 2007; Koman und } \\
\text { Sanders 1999; Letts et } \\
\text { al. 2000; Lipscomb } \\
\text { und Anderson 1986; } \\
\text { Lo et al. 1997; Matava } \\
\text { und Siegel 1997; } \\
\text { McIntosh et al. 2006; } \\
\text { Moksnes et al. 2008; } \\
\text { Parker et al. 1994; } \\
\text { Seon et al. 2005) }\end{array}$ & $\begin{array}{l}\text { konnte nicht } \\
\text { berechnet werden }\end{array}$ & $\begin{array}{l}\text { 10,7 }(2-15) \\
\text { (Kocher et al. } \\
2005 ; \text { Micheli } \\
\text { et al. 1999; } \\
\text { Nakhostine et } \\
\text { al. 1995) }\end{array}$ & $\begin{array}{l}\text { 13,6 }(9-17) \\
\text { (Andrews et } \\
\text { al. 1994; } \\
\text { Aronowitz et } \\
\text { al. 2000; } \\
\text { Fuchs et al. } \\
\text { 2002; Gorin et } \\
\text { al. 2003; } \\
\text { Sankar et al. } \\
\text { 2006; } \\
\text { Vaquero et al. } \\
\text { 2005) }\end{array}$ \\
\hline $\begin{array}{l}\text { Klinisches } \\
\text { Ergebnis } \\
\text { (Lysholm- } \\
\text { Score) }\end{array}$ & $\begin{array}{l}93,5(89-100) \\
\text { (Edwards und } \\
\text { Grana 2001; } \\
\text { Gaulrapp und } \\
\text { Haus 2003; } \\
\text { Knorr et al. } \\
\text { 2006; Lukas et } \\
\text { al. 2007; Reilly } \\
\text { und Choit 2007) }\end{array}$ & $\begin{array}{l}\text { 93,9 (89-100) } \\
\text { (Attmanspacher et al. } \\
\text { 2002; Edwards und } \\
\text { Grana 2001; Gaulrapp } \\
\text { und Haus 2006; } \\
\text { Hoffmann et al. 1998; } \\
\text { Kocher et al. 2007; } \\
\text { McIntosh et al. 2006; } \\
\text { Moksnes et al. 2008; } \\
\text { Parker et al. 1994; } \\
\text { Seon et al. 2005) }\end{array}$ & $\begin{array}{l}\text { konnte nicht } \\
\text { berechnet werden }\end{array}$ & $\begin{array}{l}96,0(74- \\
100) \\
\text { (Kocher et al. } \\
\text { 2005; Micheli } \\
\text { et al. 1999) }\end{array}$ & $\begin{array}{l}96,7 \text { (91-100) } \\
\text { (Aronowitz et } \\
\text { al. 2000; } \\
\text { Fuchs et al. } \\
\text { 2002; Sankar } \\
\text { et al. 2006) }\end{array}$ \\
\hline Rerupturrate & $\begin{array}{l}5 \text { von } 200 \\
(2,5 \%) \\
\text { (Edwards und } \\
\text { Grana 2001; } \\
\text { McCarroll et al. } \\
\text { 1994; } \\
\text { Shelbourne et } \\
\text { al. 2004) }\end{array}$ & $\begin{array}{l}16 \text { von } 359(4,5 \%) \\
\text { (Aichroth et al. 2002; } \\
\text { Bisson et al. 1998; } \\
\text { Edwards und Grana } \\
\text { 2001; Fankhauser et al. } \\
\text { 2004; Gaulrapp und } \\
\text { Haus 2006; Kocher et } \\
\text { al. 2007; Matava und } \\
\text { Siegel 1997; McIntosh } \\
\text { et al. 2006; Moksnes et } \\
\text { al. 2008; Sobau und } \\
\text { Ellermann 2004) }\end{array}$ & $\begin{array}{l}3 \text { von } 14(21,4 \%) \\
\text { (Gebhard et al. } \\
2006)\end{array}$ & $\begin{array}{l}3 \text { von } 79 \\
(3,8 \%) \\
\text { (Kocher et al. } \\
2005 ; \\
\text { McCarroll et } \\
\text { al. } 1988)\end{array}$ & $\begin{array}{l}2 \text { von } 76 \\
(2,6 \%) \\
\text { (Andrews et } \\
\text { al. 1994; } \\
\text { Vaquero et al. } \\
2005 \text { ) }\end{array}$ \\
\hline
\end{tabular}


3. Ergebnisse

\begin{tabular}{|c|c|c|c|c|c|}
\hline $\begin{array}{l}\text { Beinlängen- } \\
\text { differenzen } \\
\text { oder Achsen- } \\
\text { abweichungen }\end{array}$ & $\begin{array}{l}5 \text { von } 200 \\
(2,5 \%) \\
(2 \times \text { Genu } \\
\text { valgum, } 1 \text { x } \\
\text { Genu varum, } 2 \\
\text { x Genu } \\
\text { recurvatum) } \\
\text { (Seil und Robert } \\
2005)\end{array}$ & $\begin{array}{l}16 \text { von } 359(4,5 \%) \\
(14 \text { x } \\
\text { Beinlängendifferenz, } \\
2 \text { x Genu valgum) } \\
\text { (Anderson 2003; } \\
\text { Edwards und Grana } \\
2001 \text {; Koman und } \\
\text { Sanders 1999; } \\
\text { Lipscomb und } \\
\text { Anderson } 1986 ; \\
\text { McIntosh et al. 2006; } \\
\text { Parker et al. 1994; } \\
\text { Seon et al. 2005) }\end{array}$ & 0 von $14(0 \%)$ & $\begin{array}{l}6 \text { von } 79 \\
(7,6 \%) \\
(1 \mathrm{x} \\
\text { Beinlängen- } \\
\text { differenz, } 5 \mathrm{x} \\
\text { Genu } \\
\text { recurvatum) } \\
\text { (Micheli et al. } \\
\text { 1999; } \\
\text { Nakhostine et } \\
\text { al. 1995) }\end{array}$ & $\begin{array}{l}2 \text { von } 76 \\
(2,6 \%) \\
(2 x \\
\text { Beinlängen- } \\
\text { differenz) } \\
\text { (Andrews et } \\
\text { al. 1994) }\end{array}$ \\
\hline
\end{tabular}

Tabelle 9: Kreuzbandplastik im Wachstumsalter (bis maximal zwölf Jahre bei OP): Femoral und tibial gelenkferne versus beidseitig gelenknahe Transplantatfixierung

\begin{tabular}{|c|c|c|}
\hline & Beidseitig gelenknahe Fixierung & Beidseitig gelenkferne Fixierung \\
\hline $\begin{array}{l}\text { Anzahl der Artikel, die } \\
\text { in die Auswertung } \\
\text { eingebunden werden } \\
\text { konnten }\end{array}$ & $\begin{array}{l}3 \\
\text { (Edwards und Grana 2001; Knorr et al. } \\
\text { 2006; Robert und Bonnard 1999) }\end{array}$ & $\begin{array}{l}8 \\
\text { (Aronowitz et al. 2000; Lipscomb und } \\
\text { Anderson 1986; Lo et al. 1997; Lukas et al. } \\
\text { 2007; Matava und Siegel 1997; Micheli et al. } \\
\text { 1999; Nakhostine et al. 1995; Sankar et al. } \\
\text { 2006) }\end{array}$ \\
\hline Anzahl der Patienten & 8 & 23 \\
\hline $\begin{array}{l}\text { Durchschnittsalter } \\
\text { (Jahre) }\end{array}$ & $\begin{array}{l}10,1(5-12) \\
\text { (Edwards und Grana 2001; Knorr et al. } \\
\text { 2006; Robert und Bonnard 1999) }\end{array}$ & $\begin{array}{l}\text { 10,8 }(2-12) \\
\text { (Aronowitz et al. 2000; Lipscomb und } \\
\text { Anderson 1986; Lo et al. 1997; Lukas et al. } \\
\text { 2007; Matava und Siegel 1997; Micheli et al. } \\
\text { 1999; Nakhostine et al. 1995; Sankar et al. } \\
\text { 2006) }\end{array}$ \\
\hline $\begin{array}{l}\text { Klinisches Ergebnis } \\
\text { (Lysholm-Score) }\end{array}$ & $\begin{array}{l}94,5(90-99) \\
\text { (Edwards und Grana 2001; Knorr et al. } \\
\text { 2006) }\end{array}$ & $\begin{array}{l}\text { 96,5 (90 - 100) } \\
\text { (Aronowitz et al. 2000; Lukas et al. 2007; } \\
\text { Matava und Siegel 1997; McIntosh et al. } \\
\text { 2006; Micheli et al. 1999; Sankar et al. 2006) }\end{array}$ \\
\hline Rerupturrate & 0 von $8(0 \%)$ & 0 von $23(0 \%)$ \\
\hline $\begin{array}{l}\text { Beinlängendifferenzen } \\
\text { oder } \\
\text { Achsenabweichungen }\end{array}$ & 0 von $8(0 \%)$ & $\begin{array}{l}2 \text { von } 18(11,1 \%) \\
(1 \text { x Beinlängendifferenz, } 1 \text { x Genu } \\
\text { recurvatum) } \\
\text { (Micheli et al. 1999; Nakhostine et al. 1995) }\end{array}$ \\
\hline
\end{tabular}




\section{Tabelle 10: Kreuzbandplastik im Wachstumsalter (altersunabhängig): Femoral und tibial gelenkferne versus beidseits gelenknahe Transplantatfixierung}

\begin{tabular}{|c|c|c|}
\hline & Beidseitig gelenknahe Fixierung & Beidseitig gelenkferne Fixierung \\
\hline $\begin{array}{l}\text { Anzahl der Artikel, die } \\
\text { in die Auswertung } \\
\text { eingebunden werden } \\
\text { konnten }\end{array}$ & $\begin{array}{l}7 \\
\text { (Arbes et al. 2007; Benedetto et al. } \\
\text { 1996; Edwards und Grana 2001; } \\
\text { Fankhauser et al. 2004; Knorr et al. } \\
\text { 2006; Robert und Bonnard 1999; } \\
\text { Vaquero et al. 2005) }\end{array}$ & $\begin{array}{l}31 \\
\text { (Aichroth et al. 2002; Anderson 2003; Andrews } \\
\text { et al. 1994; Aronowitz et al. 2000; } \\
\text { Attmanspacher et al. 2002; Attmanspacher et al. } \\
\text { 2003; Bisson et al. 1998; Brief 1991; Casper et } \\
\text { al. 2006; Fuchs et al. 2002; Gaulrapp und Haus } \\
\text { 2006; Gebhard et al. 2006; Guzzanti et al. } \\
\text { 2003a; Hoffmann et al. 1998; Janarv et al. 1996; } \\
\text { Kocher et al. 2005; Kocher et al. 2007; Letts et } \\
\text { al. 2000; Lipscomb und Anderson 1986; Lo et } \\
\text { al. 1997; Lukas et al. 2007; Matava und Siegel } \\
\text { 1997; McIntosh et al. 2006; Micheli et al. 1999; } \\
\text { Nakhostine et al. 1995; Reilly und Choit 2007; } \\
\text { Sankar et al. 2006; Seon et al. 2005; Shelbourne } \\
\text { et al. 2004; Sobau und Ellermann 2004; } \\
\text { Thompson et al. 2006) }\end{array}$ \\
\hline Anzahl der Patienten & 56 & 506 \\
\hline $\begin{array}{l}\text { Durchschnittsalter } \\
\text { (Jahre) }\end{array}$ & $\begin{array}{l}\text { 13,1 }(5-17) \\
\text { (Edwards und Grana 2001; } \\
\text { Fankhauser et al. 2004; Knorr et al. } \\
\text { 2006; Robert und Bonnard 1999; } \\
\text { Vaquero et al. 2005) }\end{array}$ & $\begin{array}{l}\text { 13,2 (2-17) } \\
\text { (Aichroth et al. 2002; Anderson 2003; Andrews } \\
\text { et al. 1994; Aronowitz et al. 2000; } \\
\text { Attmanspacher et al. 2002; Brief 1991; Casper } \\
\text { et al. 2006; Fuchs et al. 2002; Gebhard et al. } \\
\text { 2006; Guzzanti et al. 2003a; Janarv et al. 1996; } \\
\text { Kocher et al. 2005; Kocher et al. 2007; Letts et } \\
\text { al. 2000; Lipscomb und Anderson 1986; Lo et } \\
\text { al. 1997; Lukas et al. 2007; Matava und Siegel } \\
\text { 1997; McIntosh et al. 2006; Micheli et al. 1999; } \\
\text { Nakhostine et al. 1995; Reilly und Choit 2007; } \\
\text { Sankar et al. 2006; Shelbourne et al. 2004) }\end{array}$ \\
\hline $\begin{array}{l}\text { Klinisches Ergebnis } \\
\text { (Lysholm-Score) }\end{array}$ & $\begin{array}{l}97,9(90-100) \\
\text { (Edwards und Grana 2001; Knorr et } \\
\text { al. 2006) }\end{array}$ & $\begin{array}{l}94,5(74-100) \\
\text { (Aronowitz et al. 2000; Attmanspacher et al. } \\
\text { 2002; Fuchs et al. 2002; Gaulrapp und Haus } \\
\text { 2006; Gebhard et al. 2006; Hoffmann et al. } \\
\text { 1998; Janarv et al. 1996; Kocher et al. 2005; } \\
\text { Kocher et al. 2007; Lukas et al. 2007; McIntosh } \\
\text { et al. 2006; Micheli et al. 1999; Reilly und } \\
\text { Choit 2007; Sankar et al. 2006) }\end{array}$ \\
\hline Rerupturrate & $\begin{array}{l}4 \text { von } 56(7,1 \%) \\
\text { (Edwards und Grana 2001; } \\
\text { Fankhauser et al. 2004; Vaquero et } \\
\text { al. 2005) }\end{array}$ & $\begin{array}{l}21 \text { von } 506(4,2 \%) \\
\text { (Aichroth et al. 2002; Andrews et al. 1994; } \\
\text { Bisson et al. 1998; Gaulrapp und Haus 2006; } \\
\text { Gebhard et al. 2006; Kocher et al. 2005; Kocher } \\
\text { et al. 2007; Matava und Siegel 1997; McIntosh } \\
\text { et al. 2006; Shelbourne et al. 2004; Sobau und } \\
\text { Ellermann 2004; Thompson et al. 2006) }\end{array}$ \\
\hline
\end{tabular}




\begin{tabular}{|l|l|l|}
\hline $\begin{array}{l}\text { Beinlängendifferenzen } \\
\text { oder }\end{array}$ & $\begin{array}{l}1 \text { von 56 (1,8\%) } \\
\text { Achsenabweichungen }\end{array}$ & $\begin{array}{l}13 \text { von } 506(2,6 \%) \\
\text { (1 x Beinlängendifferenz) }\end{array}$ \\
& $\begin{array}{l}\text { (Edwards und Grana 2001) } \\
\text { Genu recurvatum) } \\
\text { (Anderson 2003; Andrews et al. 1994; } \\
\text { Lipscomb und Anderson 1986; McIntosh et al. } \\
\text { 2006; Micheli et al. 1999; Nakhostine et al. } \\
1995)\end{array}$ \\
\hline
\end{tabular}

Tabelle 11: Kreuzbandplastik im Wachstumsalter (bis maximal zwölf Jahre bei OP): Anlage des femoralen Bohrkanals über das anteromediale Portal gegenüber einer transtibialen Anlage des Bohrkanals

\begin{tabular}{|l|l|l|}
\hline & $\begin{array}{l}\text { Anlage des femoralen Kanals über } \\
\text { anteromediales Portal }\end{array}$ & $\begin{array}{l}\text { Transtibiale Anlage des femoralen } \\
\text { Kanals }\end{array}$ \\
\hline $\begin{array}{l}\text { Anzahl der Artikel, die in die } \\
\text { kuswertung eingebunden werden } \\
\text { konnten }\end{array}$ & $\begin{array}{l}\text { (Lukas et al. 2007; Robert und } \\
\text { Bonnard 1999) }\end{array}$ & $\begin{array}{l}1 \\
\text { (Sankar et al. 2006) }\end{array}$ \\
\hline Anzahl der Patienten & 11 & 3 \\
\hline Durchschnittsalter (Jahre) & $\begin{array}{l}10,8(9-12) \\
(\text { Lukas et al. 2007; Robert und } \\
\text { Bonnard 1999) }\end{array}$ & $\begin{array}{l}\text { (Sankar et al. 2006) } \\
11,7(11-12)\end{array}$ \\
\hline $\begin{array}{l}\text { Klinisches Ergebnis (Lysholm- } \\
\text { Score) }\end{array}$ & $\begin{array}{l}96,0(90-100) \\
(\text { Lukas et al. 2007) }\end{array}$ & $\begin{array}{l}97,3(96-100) \\
\text { (Sankar et al. 2006) }\end{array}$ \\
\hline Rerupturrate & 0 von 11 (0\%) & von 3 (0\%) \\
\hline $\begin{array}{l}\text { Beinlängendifferenzen oder } \\
\text { Achsenabweichungen }\end{array}$ & von 11 (0\%) & von $)$ \\
\hline
\end{tabular}

Tabelle 12: Kreuzbandplastik im Wachstumsalter (altersunabhängig): Anlage des femoralen Bohrkanals über das anteromediale Portal gegenüber einer transtibialen Anlage des Bohrkanals

\begin{tabular}{|l|l|l|}
\hline & $\begin{array}{l}\text { Anlage des femoralen Kanals über } \\
\text { anteromediales Portal }\end{array}$ & $\begin{array}{l}\text { Transtibiale Anlage des femoralen } \\
\text { Kanals }\end{array}$ \\
\hline $\begin{array}{l}\text { Anzahl der Artikel, die in die } \\
\text { Auswertung eingebunden } \\
\text { werden konnten }\end{array}$ & $\begin{array}{l}\text { (Aichroth et al. 2002; Anderson } \\
\text { 2003; Hoffmann et al. 1998; Lukas } \\
\text { et al. 2007; Robert und Bonnard } \\
1999)\end{array}$ & $\begin{array}{l}\text { Attmanspacher et al. 2002; } \\
\text { Attmanspacher et al. 2003; Kocher et al. } \\
\text { al. 2006; Thompson et al. 2006) }\end{array}$ \\
\hline
\end{tabular}




\begin{tabular}{|c|c|c|}
\hline Anzahl der Patienten & 94 & 141 \\
\hline Durchschnittsalter (Jahre) & $\begin{array}{l}\text { 13,0 }(9-16) \\
\text { (Aichroth et al. 2002; Anderson } \\
\text { 2003; Lukas et al. 2007; Robert und } \\
\text { Bonnard 1999) }\end{array}$ & $\begin{array}{l}\text { 14,0 (10 - 16) } \\
\text { (Attmanspacher et al. 2002; } \\
\text { Attmanspacher et al. 2003; Kocher et al. } \\
\text { 2007; McIntosh et al. 2006; Sankar et } \\
\text { al. 2006) }\end{array}$ \\
\hline $\begin{array}{l}\text { Klinisches Ergebnis (Lysholm- } \\
\text { Score) }\end{array}$ & $\begin{array}{l}96,1(89-100) \\
\text { (Hoffmann et al. 1998; Lukas et al. } \\
\text { 2007) }\end{array}$ & $\begin{array}{l}93,9(89-100) \\
\text { (Attmanspacher et al. 2002; Kocher et } \\
\text { al. 2007; McIntosh et al. 2006; Sankar } \\
\text { et al. 2006) }\end{array}$ \\
\hline Rerupturrate & $\begin{array}{l}3 \text { von } 94(3,2 \%) \\
\text { (Aichroth et al. 2002) }\end{array}$ & $\begin{array}{l}5 \text { von } 141(3,5 \%) \\
\text { (Kocher et al. 2007; McIntosh et al. } \\
\text { 2006; Thompson et al. 2006) }\end{array}$ \\
\hline $\begin{array}{l}\text { Beinlängendifferenzen oder } \\
\text { Achsenabweichungen }\end{array}$ & $\begin{array}{l}1 \text { von } 94(1,1 \%) \\
(1 \text { x Beinlängendifferenz) } \\
\text { (Anderson 2003) }\end{array}$ & $\begin{array}{l}1 \text { von } 141(0,7 \%) \\
(1 \times \text { Beinlängendifferenz) } \\
\text { (McIntosh et al. 2006) }\end{array}$ \\
\hline
\end{tabular}

Tabelle 13: Analyse der Beinlängendifferenzen oder Achsenabweichungen nach Kreuzbandplastik im Wachstumsalter: Vergleich der Altersgruppen

\begin{tabular}{|c|c|c|c|}
\hline & $\begin{array}{l}\text { Bis maximal zwölf } \\
\text { Jahre bei OP }\end{array}$ & Ab 13 Jahre bei OP & Altersunabhängig \\
\hline $\begin{array}{l}\text { Beinlängendifferenzen } \\
\text { oder } \\
\text { Achsenabweichungen } \\
\text { insgesamt }\end{array}$ & $\begin{array}{l}2 \text { von } 37(5,4 \%) \\
\text { (Micheli et al. 1999; } \\
\text { Nakhostine et al. } \\
1995 \text { ) }\end{array}$ & $\begin{array}{l}10 \text { von } 262(3,8 \%) \\
\text { (Edwards und Grana } \\
\text { 2001; Koman und } \\
\text { Sanders 1999; } \\
\text { Nakhostine et al. 1995; } \\
\text { Seon et al. 2005) }\end{array}$ & $\begin{array}{l}29 \text { von } 769(3,8 \%) \\
\text { (Anderson 2003; Andrews et al. } \\
\text { 1994; Edwards und Grana 2001; } \\
\text { Koman und Sanders 1999; Lipscomb } \\
\text { und Anderson 1986; McIntosh et al. } \\
\text { 2006; Micheli et al. 1999; } \\
\text { Nakhostine et al. 1995; Parker et al. } \\
\text { 1994; Seil und Robert 2005; Seon et } \\
\text { al. 2005) }\end{array}$ \\
\hline Operiertes Bein länger & 0 von $37(0 \%)$ & $\begin{array}{l}3 \text { von } 262(1,1 \%) \\
(\text { Seon et al. 2005) }\end{array}$ & $\begin{array}{l}12 \text { von } 769(1,6 \%) \\
\text { (Anderson 2003; Andrews et al. } \\
\text { 1994; Lipscomb und Anderson 1986; } \\
\text { McIntosh et al. 2006; Parker et al. } \\
\text { 1994; Seon et al. 2005) }\end{array}$ \\
\hline Operiertes Bein kürzer & $\begin{array}{l}1 \text { von } 37(2,7 \%) \\
\text { (Micheli et al. 1999) }\end{array}$ & $\begin{array}{l}2 \text { von } 262(0,8 \%) \\
\text { (Edwards und Grana } \\
\text { 2001; Seon et al. 2005) }\end{array}$ & $\begin{array}{l}5 \text { von } 769(0,7 \%) \\
\text { (Andrews et al. 1994; Edwards und } \\
\text { Grana 2001; Lipscomb und Anderson } \\
\text { 1986; Micheli et al. 1999; Seon et al. } \\
\text { 2005) }\end{array}$ \\
\hline
\end{tabular}




\begin{tabular}{|c|c|c|c|}
\hline Genu valgum & 0 von $37(0 \%)$ & $\begin{array}{l}1 \text { von } 262(0,4 \%) \\
\text { (Koman und Sanders } \\
1999)\end{array}$ & $\begin{array}{l}4 \text { von } 769(0,5 \%) \\
\text { (Koman und Sanders 1999; } \\
\text { Lipscomb und Anderson 1986; Seil } \\
\text { und Robert 2005) }\end{array}$ \\
\hline Genu varum & 0 von $37(0 \%)$ & 0 von $262(0 \%)$ & $\begin{array}{l}1 \text { von } 769(0,1 \%) \\
\text { (Seil und Robert 2005) }\end{array}$ \\
\hline Genu recurvatum & $\begin{array}{l}1 \text { von } 37(2,7 \%) \\
\text { (Nakhostine et al. } \\
1995)\end{array}$ & $\begin{array}{l}4 \text { von } 262(1,5 \%) \\
\text { (Nakhostine et al. 1995) }\end{array}$ & $\begin{array}{l}7 \text { von } 769(0,9 \%) \\
\text { (Nakhostine et al. 1995; Seil und } \\
\text { Robert 2005) }\end{array}$ \\
\hline
\end{tabular}

Tabelle 14: Kreuzbandnaht: Therapieergebnisse bei Patienten bis zu einem Alter von zwölf Jahren gegenüber Patienten ab 13 Jahren zum Zeitpunkt der OP

\begin{tabular}{|c|c|c|}
\hline & Patienten bis zwölf Jahre & Patienten ab 13 Jahre \\
\hline $\begin{array}{l}\text { Anzahl der Artikel, die in die } \\
\text { Auswertung eingebunden } \\
\text { werden konnten }\end{array}$ & $\begin{array}{l}4 \\
\text { (Bradley et al. 1979; DeLee und } \\
\text { Curtis 1983; Gaudernak 1996; } \\
\text { Schaefer et al. 1993) }\end{array}$ & $\begin{array}{l}2 \\
\text { (Engebretsen et al. 1988; Gaudernak } \\
\text { 1996) }\end{array}$ \\
\hline Anzahl der Patienten & 6 & 9 \\
\hline Durchschnittsalter (Jahre) & $\begin{array}{l}9,8(4-12) \\
\text { (Bradley et al. 1979; DeLee und } \\
\text { Curtis 1983; Gaudernak 1996; } \\
\text { Schaefer et al. 1993) }\end{array}$ & $\begin{array}{l}14,9(13-16) \\
\text { (Engebretsen et al. 1988; Gaudernak } \\
\text { 1996) }\end{array}$ \\
\hline $\begin{array}{l}\text { Klinisches Ergebnis (Lysholm- } \\
\text { Score) }\end{array}$ & k.A. & k.A. \\
\hline Rerupturrate & $\begin{array}{l}2 \text { von } 6(33,3 \%) \\
\text { (Gaudernak 1996; Schaefer et al. } \\
\text { 1993) }\end{array}$ & $\begin{array}{l}1 \text { von } 9(11,1 \%) \\
\text { (Gaudernak 1996) }\end{array}$ \\
\hline $\begin{array}{l}\text { Beinlängendifferenzen oder } \\
\text { Achsenabweichungen }\end{array}$ & 0 von $6(0 \%)$ & 0 von $9(0 \%)$ \\
\hline
\end{tabular}




\section{Tabelle 15: Kreuzbandnaht versus Kreuzbandplastik bei Patienten im Wachstumsalter (altersunabhängig)}

\begin{tabular}{|c|c|c|}
\hline & Bandnaht & Bandplastik \\
\hline $\begin{array}{l}\text { Anzahl der Artikel, die } \\
\text { in die Auswertung } \\
\text { eingebunden werden } \\
\text { konnten }\end{array}$ & $\begin{array}{l}14 \\
\text { (Arbes et al. 2007; } \\
\text { Arndt et al. 1999; } \\
\text { Attmanspacher et al. } \\
\text { 2003; Bradley et al. } \\
\text { 1979; DeLee und } \\
\text { Curtis 1983; } \\
\text { Engebretsen et al. } \\
\text { 1988; Gaudernak } \\
\text { 1996; Gaulrapp und } \\
\text { Haus 2006; Kunz M } \\
\text { 1992; Millett et al. } \\
\text { 2002; Pressman et al. } \\
\text { 1997; Schaefer et al. } \\
\text { 1993; Schultz et al. } \\
\text { 1996; Wahler et al. } \\
\text { 1998) }\end{array}$ & $\begin{array}{l}\text { 52 } \\
\text { (Aichroth et al. 2002; Anderson 2003; Andrews et al. 1994; Arbes } \\
\text { et al. 2007; Arndt et al. 1999; Aronowitz et al. 2000; } \\
\text { Attmanspacher et al. 2002; Attmanspacher et al. 2003; Benedetto } \\
\text { et al. 1996; Bisson et al. 1998; Bollen et al. 2008; Brief 1991; } \\
\text { Casper et al. 2006; Edwards und Grana 2001; Fankhauser et al. } \\
\text { 2004; Fuchs et al. 2002; Gaulrapp und Haus 2003; Gaulrapp und } \\
\text { Haus 2006; Gebhard et al. 2006; Gorin et al. 2003; Guzzanti et al. } \\
\text { 2003b; Guzzanti et al. 2003a; Hoffmann et al. 1998; Janarv et al. } \\
\text { 1996; Knorr et al. 2006; Kocher et al. 2005; Kocher et al. 2007; } \\
\text { Koman und Sanders 1999; Letts et al. 2000; Lipscomb und } \\
\text { Anderson 1986; Lo et al. 1997; Lukas et al. 2007; Matava und } \\
\text { Siegel 1997; McCarroll et al. 1988; McCarroll et al. 1994; } \\
\text { McIntosh et al. 2006; Micheli et al. 1999; Millett et al. 2002; } \\
\text { Moksnes et al. 2008; Nakhostine et al. 1995; Parker et al. 1994; } \\
\text { Pressman et al. 1997; Reilly und Choit 2007; Robert und Bonnard } \\
\text { 1999; Sankar et al. 2006; Seil und Robert 2005; Seon et al. 2005; } \\
\text { Shelbourne et al. 2004; Sobau und Ellermann 2004; Thompson et } \\
\text { al. 2006; Vaquero et al. 2005; Wahler et al. 1998) }\end{array}$ \\
\hline Anzahl der Patienten & 91 & 769 \\
\hline $\begin{array}{l}\text { Durchschnittsalter } \\
\text { (Jahre) }\end{array}$ & $\begin{array}{l}\text { 12,8 }(4-16) \\
\text { (Bradley et al. 1979; } \\
\text { DeLee und Curtis } \\
\text { 1983; Engebretsen et } \\
\text { al. 1988; Gaudernak } \\
\text { 1996; Pressman et al. } \\
\text { 1997; Schaefer et al. } \\
\text { 1993) }\end{array}$ & $\begin{array}{l}\text { 13,4 }(2-17) \\
\text { (Aichroth et al. 2002; Anderson 2003; Andrews et al. 1994; } \\
\text { Aronowitz et al. 2000; Attmanspacher et al. 2002; Bollen et al. } \\
\text { 2008; Brief 1991; Casper et al. 2006; Edwards und Grana 2001; } \\
\text { Fankhauser et al. 2004; Fuchs et al. 2002; Gebhard et al. 2006; } \\
\text { Gorin et al. 2003; Guzzanti et al. 2003b; Guzzanti et al. 2003a; } \\
\text { Janarv et al. 1996; Knorr et al. 2006; Kocher et al. 2005; Kocher } \\
\text { et al. 2007; Koman und Sanders 1999; Letts et al. 2000; } \\
\text { Lipscomb und Anderson 1986; Lo et al. 1997; Lukas et al. 2007; } \\
\text { Matava und Siegel 1997; McCarroll et al. 1994; McIntosh et al. } \\
\text { 2006; Micheli et al. 1999; Moksnes et al. 2008; Nakhostine et al. } \\
\text { 1995; Parker et al. 1994; Reilly und Choit 2007; Robert und } \\
\text { Bonnard 1999; Sankar et al. 2006; Seon et al. 2005; Shelbourne et } \\
\text { al. 2004; Vaquero et al. 2005; Wahler et al. 1998) }\end{array}$ \\
\hline $\begin{array}{l}\text { Klinisches Ergebnis } \\
\text { (Lysholm-Score) }\end{array}$ & $\begin{array}{l}\text { 79,6 (Minimum und } \\
\text { Maximum nicht } \\
\text { angegeben) } \\
\text { (Gaulrapp und Haus } \\
\text { 2006; Pressman et al. } \\
\text { 1997) }\end{array}$ & $\begin{array}{l}\text { 94,4 (62 - 100) } \\
\text { (Aronowitz et al. 2000; Attmanspacher et al. 2002; Edwards und } \\
\text { Grana 2001; Fuchs et al. 2002; Gaulrapp und Haus 2003; } \\
\text { Gaulrapp und Haus 2006; Hoffmann et al. 1998; Janarv et al. } \\
\text { 1996; Knorr et al. 2006; Kocher et al. 2005; Kocher et al. 2007; } \\
\text { Lukas et al. 2007; McIntosh et al. 2006; Micheli et al. 1999; } \\
\text { Moksnes et al. 2008; Parker et al. 1994; Reilly und Choit 2007; } \\
\text { Sankar et al. 2006; Seon et al. 2005) }\end{array}$ \\
\hline Rerupturrate & $\begin{array}{l}7 \text { von } 91(7,7 \%) \\
\text { (Gaudernak 1996; } \\
\text { Millett et al. 2002; } \\
\text { Schaefer et al. } 1993\end{array}$ & $\begin{array}{l}30 \text { von } 769(3,9 \%) \\
\text { (Aichroth et al. 2002; Andrews et al. 1994; Bisson et al. 1998; } \\
\text { Edwards und Grana 2001; Fankhauser et al. 2004; Gaulrapp und } \\
\text { Haus 2006; Gebhard et al. 2006; Kocher et al. 2005; Kocher et al. }\end{array}$ \\
\hline
\end{tabular}




\begin{tabular}{|l|l|l|}
\hline & Wahler et al. 1998) & $\begin{array}{l}\text { 2007; Matava und Siegel 1997; McCarroll et al. 1988; McCarroll } \\
\text { et al. 1994; McIntosh et al. 2006; Moksnes et al. 2008; } \\
\text { Shelbourne et al. 2004; Sobau und Ellermann 2004; Thompson et } \\
\text { al. 2006; Vaquero et al. 2005) }\end{array}$ \\
\hline $\begin{array}{l}\text { Beinlängendifferenzen } \\
\text { oder }\end{array}$ & Achsenabweichungen & $\begin{array}{l}\text { 29 von 769 (3,8\%) } \\
\text { (17x Beinlängendifferenz, } 4 \text { x Genu valgum, 1 x Genu varum, 7 x } \\
\text { Genu recurvatum) } \\
\text { (Anderson 2003; Andrews et al. 1994; Edwards und Grana 2001; } \\
\text { Koman und Sanders 1999; Lipscomb und Anderson 1986; } \\
\text { McIntosh et al. 2006; Micheli et al. 1999; Nakhostine et al. 1995; } \\
\text { Parker et al. 1994; Seil und Robert 2005; Seon et al. 2005) }\end{array}$ \\
\hline
\end{tabular}




\section{Diskussion}

In der vorliegenden Arbeit wurde einerseits eine Multicenterstudie zur VKB-Plastik bei Patienten bis zu einem Alter von zwölf Jahren zum OP-Zeitpunkt durchgeführt sowie andererseits eine systematische Analyse der aktuellen Literatur zur VKB-Plastik und VKBNaht bei Patienten bis zu einem Alter von zwölf Jahren ausgearbeitet. Zum Vergleich wurden Daten von Patienten jeden Alters bei noch offenen Wachstumsfugen nach Versorgung einer VKB-Ruptur bzw. für eine Fragestellung Patienten in einem Alter von mindestens 13 Jahren mit noch offenen Wachstumsfugen herangezogen.

Als systematisch werden Literaturanalysen dann bezeichnet, wenn sie vordefinierte und transparente Methoden bezüglich Literatursuche, Studienselektion und Studienauswertung verwenden (Cook et al. 1997). Eine Meta-Analyse der Literatur war nicht durchführbar, da sie eine Auswertung in Form einer integrierenden statistischen Analyse aller eingeschlossenen Studien wie eine einzige große Studie (pooling) vorraussetzt (Linde 2002). Dies war aufgrund der großen Heterogenität nicht möglich.

Die VKB-Ruptur ist bei Kindern und Jugendlichen insgesamt selten (Andrish 2001; Lipscomb und Anderson 1986). Dies gilt umso mehr für junge Patienten im Alter von bis zu zwölf Jahren. Je jünger die Patienten sind, desto höher ist die Wahrscheinlichkeit von epiphysären Ausrissfrakturen relativ zu der Ruptur des VKB (Rinaldi und Mazzarella 1980, Seil, 2002). Dies ist darauf zurückzuführen, dass die kollagenen Fasern des VKB in das Perichondrium des epiphysären Knorpels übergehen, welcher im allgemeinen bei Patienten im Wachstumsalter weniger reißfest ist als das VKB selbst (Micheli und Coady 1997). So konnten Kellenberger und von Laer (1990) in einer Untersuchung von 62 Patienten im Wachstumsalter mit einer VKB-Ruptur zeigen, dass es bei jüngeren Patienten vorwiegend zu knöchernen Ausrissverletzungen kommt, bei älteren hingegen zu ligamentären Rupturen. 80 Prozent der untersuchten Patienten, die jünger waren als zwölf Jahre, erlitten einen knöchernen Bandausriss, bei 90 Prozent der Patienten, die älter waren als zwölf Jahre, diagnostizierten die Untersucher hingegen eine intraligamentäre Ruptur.

Dementsprechend sind auch die publizierten Daten und das Wissen zur VKB-Ruptur bei Patienten mit einem Alter von bis zu zwölf Jahren sehr begrenzt (Moksnes et al. 2008). Daher wurde diese Altersgrenze als Einschlusskriterium für die vorliegende Studie gewählt.

In der Multicenterstudie konnten 25 Patienten aus zehn Zentren eingeschlossen werden. Berechnungen auf Basis dieses kleinen Patientenkollektivs haben eine eingeschränkte 
statistische Aussagekraft. Da zum Beispiel bei den 25 Patienten insgesamt zwölf verschiedene Methoden der Transplantatfixierung zur Anwendung kamen, war eine einzelne Auswertung dieser Methoden wenig sinnvoll. Die Bewertung der Größe des Patientenkollektivs muss jedoch vor dem Hintergrund stattfinden, dass eine ligamentäre Ruptur des VKB bei Patienten bis zu zwölf Jahren extrem selten ist (DeLee und Curtis 1983; Seil und Robert 2005). Auch in Anbetracht der Tatsache, dass in der gesamten publizierten Literatur nur 37 höchstens zwölf jährige Patienten mit dazugehöriger Operationsmethode und Therapieergebnis beschrieben werden, verdienen die hier aufgeführten Ergebnisse trotz eingeschränkter statistischer Aussagekraft Beachtung.

Mit Blick auf die Patientenzahl und die weitere Unterteilung in Untergruppen wurde - unter regelmäßiger Betreuung durch die Abteilung der Medizinischen Statistik der Universität Göttingen - entschieden, die Ergebnisse der Multicenterstudie rein deskriptiv und ohne die Anwendung statistischer Tests darzustellen. Das Hauptaugenmerk dieser Arbeit gilt Patienten bis zu einem Alter von zwölf Jahren. Da die aktuelle Datenlage für diese Altersgruppe jedoch sehr dünn ist, wurde die Literaturanalyse zur Beleuchtung bestimmter Fragestellungen auf Patienten im Wachstumsalter, also bis zum Verschluss der Wachstumsfugen und somit auch über ein Alter von zwölf Jahren hinaus, ausgeweitet.

\subsection{Beinlängendifferenzen und Achsenabweichungen}

Laut Moksnes et al. (2008) ist die wichtigste Frage bezüglich der optimalen Versorgung einer VKB-Ruptur bei offenen Wachstumsfugen, wie hoch das Risiko für eine Wachstumsstörung durch diese Therapie ist.

Eine Verletzung der kniegelenksnahen Wachstumsfugen durch eine Kreuzbandersatzoperation kann zu einer Epiphysiodese und einer Wachstumsstörung des operierten Beines führen (Koman und Sanders 1999; Lipscomb und Anderson 1986; Moksnes et al. 2008; Seil und Robert 2005). Ein gewisses Maß an Traumatisierung toleriert die Wachstumsfuge jedoch auch, ohne dass deren Wachstum beeinträchtigt wird (Seil und Robert 2005). Wie hoch diese Toleranz gegenüber einer Verletzung ist, ist derzeit noch nicht genau bekannt (Seil 2002). In der Literatur findet man nur wenige Studien, die über das Auftreten einer iatrogenen Wachstumsstörung berichten (Steadman et al. 2006). Dass es dennoch immer wieder zu solchen Komplikationen kommt, belegt eine Studie von Kocher et al. (2002), in der die Berichte über postoperative Wachstumsstörungen von 140 internationalen Kniespezialisten erfasst wurden. Insgesamt berichtet die Studie von 15 skelettalen 
Deformierungen, die auf eine VKB Rekonstruktion zurückzuführen sind. Bei zehn Patienten mit offenen Wachstumsfugen zum Zeitpunkt der Operation war es zu einer Achsenabweichung in Form einer Valgus-Fehlstellung des Kniegelenks gekommen, dreimal trat eine Achsenabweichung in Form eines Genu recurvatum auf. Zwei Patienten wiesen eine Beinlängendifferenz auf. Bei dem einen Patient war das operierte Bein bei gleichzeitiger Valgus-Fehlstellung um 2,5 cm verkürzt, bei dem anderen durch ein übermäßiges Wachstum $3 \mathrm{~cm}$ länger als das gesunde Bein. Diese Studie stellt die bisher umfassendste Erhebung zur Häufigkeit von Wachstumsstörungen durch vordere Kreuzbandplastik bei Patienten im Wachstumsalter dar (Shea et al. 2003). Da jedoch die Anzahl der insgesamt operierten Patienten nicht angegeben wird, ist es nicht möglich, eine Aussage über das Risiko für ein Fehlwachstum zu machen. Eine Verwertung der Daten in unserer systematischen Literaturanalyse war aufgrund fehlender Einschlusskriterien nicht möglich.

\subsubsection{Tierexperimentelle Studien}

Einige tierexperimentelle Studien haben sich dem Problem der Wachstumsstörungen aufgrund von Durchbohrungen der Wachstumsfugen bei der Kreuzbandersatztherapie gewidmet.

Makela et al. (1988) durchbohrten die aktive distale femorale Wachstumsfuge von 44 Kaninchen. Sie stellten fest, dass es bei einer Zerstörung von drei Prozent der Fläche der Wachstumsfuge nicht zu einer Beeinträchtigung des normalen Wachstums kam; dass eine Zerstörung von sieben Prozent der Fläche jedoch eine dauerhafte Wachstumsstörung mit einer Verkürzung des Beines bewirkte.

Stadelmaier et al. (1995) operierten acht Hunde mit offenen Wachstumsfugen. Sie durchbohrten die distale Wachstumsfuge des Femurs sowie die proximale Wachstumsfuge der Tibia. Bei vier Hunden ersetzten sie das natürliche Kreuzband durch eine Ersatzplastik aus autologer Fascia lata, bei den vier anderen ließ man die Bohrkanäle leer. Zwei Wochen bzw. vier Monate nach der Operation wurden die Wachstumsfugen von jeweils zwei Hunden aus beiden Gruppen untersucht. Bei den Hunden, bei denen die Bohrkanäle ungefüllt geblieben waren, hatte sich schon innerhalb von zwei Wochen eine Knochenbrücke über die Wachstumsfuge ausgebildet. Keine Knochenbrücke fand sich jedoch bei den anderen vier Hunden, bei denen die Bohrkanäle mit einem autologen Fascia-lata-Transplantat gefüllt worden waren. Die Autoren schlossen daraus, dass ein Weichgewebetransplantat, welches sich im Bohrkanal auf Höhe der Wachstumsfuge befindet, verhindert, dass sich eine 
Knochenbrücke ausbilden kann, die wiederum zu einer Wachstumsstörung der Extremität führen könnte.

Guzzanti et al. (1994) berichten über Kreuzbandersatzoperationen mit einer SemitendinosusSehne bei 21 Kaninchen mit offenen Wachstumsfugen. Die Schädigung der Wachstumsfuge betrug am distalen Femur elf Prozent in Projektion auf die Frontalebene und drei Prozent der Fugenfläche, an der proximalen Tibia zwölf Prozent in Projektion auf die Frontalebene und vier Prozent der Fugenfläche. Bei der späteren Untersuchung der Versuchstiere zeigten sich femoral keine Beeinträchtigungen des normalen Wachstums. Tibial jedoch hatten sich zwei Valgusdeformitäten entwickelt. Bei einem Tier war das operierte Bein im Vergleich zur Gegenseite zusätzlich auch verkürzt. Bei der histologischen Untersuchung der Kniegelenke zeigte sich bei keiner der betroffenen Wachstumsfugen eine Verknöcherung. Auch die zwei tibialen Wachstumsfugen, welche eine Valgusdeformität verursacht hatten, waren normal weit geöffnet. Die Autoren empfehlen eine sehr genaue Evaluation des prozentualen Anteils der Wachstumsfugenverletzung bei Kreuzbandersatzoperationen bei Patienten im Wachstumsalter.

Babb et al. (2008) operierten drei Gruppen von jeweils fünf noch nicht ausgewachsenen Kaninchen. In der ersten Gruppe wurden die Wachstumsfugen des distalen Femurs und der proximalen Tibia durchbohrt und der Bohrkanal leer belassen. Bei der zweiten wurden die Fugen durchbohrt und eine Kreuzbandplastik mit autologer Sehne des Musculus extensor digitorum communis eingesetzt. Die dritte Gruppe wurde prinzipiell gleich der zweiten behandelt, mit dem Unterschied, dass das Transplantat aus der Sehne des Musculus extensor digitorum communis zusammen mit mesenchymalen Stammzellen eingepflanzt wurde. Bei der späteren Untersuchung der operierten Gelenke und Wachstumsfugen zeigte sich, dass es nur bei denjenigen Tieren, die mit mesenchymalen Stammzellen behandelt worden waren, nicht zu Knochenbrücken über die Wachstumsfugen und Achsenabweichungen der Beine gekommen war. Die Autoren schlussfolgern daraus, dass das Einbringen von mesenchymalen Stammzellen das Auftreten von Wachstumsstörungen nach einer Wachstumsfugendurchbohrung verhindern oder minimieren kann.

Edwards et al. (2001) konnten zeigen, dass eine sehr starke Transplantatspannung auch ohne das Vorhandensein von Knochenbrücken zu Wachstumsstörungen führen kann. In der Studie wurden autologe Fascia-lata-Transplantate mit 80 Newton Zug über die distale femorale und proximale tibiale Wachstumsfuge von jungen Hunden gespannt. Die hohe Transplantatspannung führte zu Knochendeformitäten sowohl auf der femoralen als auch auf 
der tibialen Seite. Der genaue Wert für die Transplantatspannung, bei dem beim Menschen Wachstumsstörungen auftreten können, ist noch unbekannt (Seil 2002).

\subsubsection{Die Grenzwerte zur Einstufung eines Fehlwachstums}

In dieser Arbeit sollten insbesondere postoperative Beinlängendifferenzen oder Achsenabweichungen nach vorderer Kreuzbandplastik erfasst und analysiert werden. Daher wurde ein Grenzwert festgelegt, der bei mindestens ein Zentimeter Beinlängendifferenz bzw. bei mindestens drei Grad Achsenabweichung zur Gegenseite in der Frontal- oder Sagittalebene lag. Ab diesen Werten wurden Abweichungen als Fehlwachstum eingestuft.

Die Grenzen für eine Beinlängendifferenz oder Achsenabweichung wurden vergleichbar niedrig gewählt, um die in der publizierten Literatur beschriebenen Fälle eines Fehlwachstums möglichst vollständig auswerten zu können. Andere Autoren setzten die Grenzen für ein Fehlwachstum mitunter höher als in der vorliegenden Arbeit. Daher konnten in der systematischen Literaturanalyse nicht alle Fälle eines Fehlwachstums, welche die hier festgelegten Grenzen überschritten, erfasst werden, da sie von einigen Autoren gar nicht beschrieben wurden, da sie ihre eigenen Grenzen höher gesetzt hatten (Gaulrapp und Haus 2003; Matava und Siegel 1997; Seon et al. 2005). Bei unserer, in der systematischen Literaturanalyse ermittelten, Rate an Beinlängendifferenzen oder Achsenabweichungen von insgesamt 29 von 769 (3,8 Prozent) bei einer Kreuzbandplastik und auch bei allen anderen berechneten Fehlwachstumsraten für die einzelnen untersuchten Untergruppen des Patientenkollektivs, ist daher von einer zusätzlichen Dunkelziffer auszugehen.

Ein Nachteil der in dieser Arbeit gesetzten Grenzen für Beinlängendifferenzen oder Achsenabweichungen ist, dass mit diesen vergleichsweise niedrigen Grenzwerten die Wahrscheinlichkeit, idiopathische Beinlängendifferenzen als pathologisches Fehlwachstum zu erfassen, steigt. Eine idiopathische Beinlängendifferenz von $11 \mathrm{~mm}$ oder mehr ist laut Rush und Steiner (1946) bei beschwerdefreien Menschen mit einer Häufigkeit von vier Prozent zu finden.

\subsubsection{Ursachenanalyse für die Beinlängendifferenzen und Achsenabweichungen}

In der Studie von Kocher et al. (2002) werden folgende Ursachen für das Entstehen der 15 beschriebenen Wachstumsstörungen genannt: Dreimal bedingte ein Implantat, mit dem das Transplantat fixiert wurde, eine Valgus-Fehlstellung (Interferenzschraube, Klammer, Transfixationsstift). Das Implantat kreuzte in allen Fällen den lateralen Bereich der distalen 
Wachstumsfuge des Femurs und verursachte eine Knochenbrücke. Dreimal verursachte der Knochenblock eines Patellasehnentransplantats eine Valgus-Fehlstellung im Bereich des distalen Femurs, da der Knochenblock im lateralen Bereich der Fuge gelegen hatte und diese dort verknöcherte. Einmal führte ein großer Tunneldurchmesser von $12 \mathrm{~mm}$ zu einer Knochenbrücke über die distale Wachstumsfuge des Femurs und verursachte so eine ValgusFehlstellung. Ein anderes Mal kam es durch die femorale over-the-top-Fixierung zu einer einseitigen Fugenverknöcherung und einer Valgus-Verformung. Zweimal führte eine laterale extraartikuläre Tenodese am Femur zu einem Genu valgum, ohne dass sich eine Knochenbrücke über die Wachstumsfuge ausgebildet hatte. Einmal bedingte das Festnähen der Sehne am tibialen Periost, zweimal eine Klammer, die die Apophyse kreuzte, eine Verknöcherung der Apophyse des tibialen Tuberkels und so ein Genu recurvatum. Die Beinlängendifferenz von minus 2,5 cm mit zusätzlicher Valgus-Fehlstellung wurde auf einen großen Bohrtunneldurchmesser von $12 \mathrm{~mm}$ zurückgeführt. Für eine Beinlängendifferenz von $3 \mathrm{~cm}$ gab es keine eindeutige Erklärung.

Die Kliniken dieser Multicenterstudie berichteten in sechs Fällen über eine Beinlängendifferenz oder Achsenabweichung. Zweimal wurde ein isoliertes Genu recurvatum, zweimal eine isolierte Beinverkürzung im Vergleich zur Gegenseite beobachtet. Ein Patient entwickelte eine relative Beinverkürzung, die zusammen mit einem Genu recurvatum auftrat. Ein weiterer wies bei der Nachuntersuchung eine relative Verkürzung des Beines, zusammen mit einem Genu recurvatum und einer Valgusabweichung auf. Nur für den letztgenannten wurde die vermutliche Ursache für die Wachstumsstörung angegeben.

In der systematischen Literaturanalyse wurden insgesamt 29 Beinlängendifferenzen oder Achsenabweichungen detektiert. 17-mal handelte es sich um eine Beinlängendifferenz, wobei das operierte Bein in zwölf Fällen länger und in fünf Fällen kürzer war als das nicht betroffene Bein. In sieben Fällen hatte sich ein Genu recurvatum entwickelt, viermal ein Genu valgum und einmal ein Genu varum. Nur für zwei dieser Patienten war ein Alter von höchstens zwölf Jahren beschrieben, das Alter der anderen lag entweder über zwölf Jahren oder war nicht angegeben. Einer dieser beiden Patienten entwickelte eine Beinverkürzung (Micheli et al. 1999), der andere ein Genu recurvatum (Nakhostine et al. 1995).

Von den insgesamt elf Autoren (Anderson 2003; Andrews et al. 1994; Edwards und Grana 2001; Koman und Sanders 1999; Lipscomb und Anderson 1986; McIntosh et al. 2006; Micheli et al. 1999; Nakhostine et al. 1995; Parker et al. 1994; Seil und Robert 2005; Seon et al. 2005), die in ihren Studien Beinlängendifferenzen oder Achsenabweichungen 
beobachteten, gaben nur zwei Autoren die vermutliche Ursache für die Entstehung der Wachstumsstörung an.

\subsubsection{Mögliche Ursachen für eine Beinlängendifferenz}

Die von einer Klinik der Multicenterstudie beschriebene Beinlängendifferenz von minus 30 mm im Vergleich zur nicht operierten Seite trat gemeinsam mit einer Deformität der Beinachse auf. Diese komplexe Wachstumsstörung wurde vom nachuntersuchenden Arzt mit einem zu weit dorsal gebohrten femoralen Knochenkanal in Verbindung gebracht, der vermutlich den Ranvierschen Schnürring verletzte und $\mathrm{zu}$ einem peripheren Epiphysiodeseeffekt führte.

Die von Lipscomb und Anderson (1986) beschriebene Beinverkürzung von $20 \mathrm{~mm}$ wurde durch Befestigungsklammern verursacht, mit denen die tibiale und die femorale Fuge blockiert worden war. Eine weitere Beinverkürzung wurde ätiologisch von den Autoren mit dem vorbestehenden Krankheitsbild einer „kongenitalen Anisomelie“ mit begleitender Kreuzbandinsuffizienz in Verbindung gebracht (Micheli et al. 1999).

Prinzipiell kann eine Verletzung der Wachstumsfuge zu einer knöchernen Überbrückung der knorpeligen Fuge führen und das Wachstum in der Folge hemmen oder zum Erliegen bringen (Fehnel und Johnson 2000). Ist die Wachstumshemmung bzw. der Wachstumsstopp symmetrisch, so wird keine Winkelabweichung entstehen, sondern das betroffene Bein im Vergleich zur Gegenseite kürzer bleiben (Seil 2002). Die Aktivität der Wachstumsfuge kann durch eine Manipulation aber nicht nur gehemmt, sondern auch aktiviert werden (von Recum et al. 2002). In diesem Fall kann eine Beinlängendifferenz mit einem im Vergleich zur Gegenseite längeren Bein die Folge sein. Denkbar wäre auch eine zu große Transplantatspannung, die zu einer relativen Verkürzung des Beines führen könnte (Edwards et al. 2001).

Von einigen Untersuchern der ausgewerteten Artikel ist die präoperative Beinlängendifferenz nicht bestimmt worden (Anderson 2003; Seon et al. 2005), so dass die Beinlängendifferenz, die zum Zeitpunkt der Nachuntersuchung bestand, nicht mit letzter Sicherheit auf die Kreuzbandoperation zurückgeführt werden kann.

\subsubsection{Mögliche Ursachen für ein Genu recurvatum}

In der Multicenterstudie wurde für keinen der vier gemeldeten Fälle eines Genu recurvatum die vermutete Ursache für deren Entstehung beschrieben. Auch in der analysierten Literatur 
machte keiner der Autoren, die bei ihren Patienten ein Genu recurvatum beobachteten, eine Angabe über welchen Mechanismus sich dieses Fehlwachstum entwickelt haben könnte.

Für die Entstehung eines Genu recurvatum sind mehrere mögliche Ursachen zu diskutieren. Zum einen könnte durch die Anlage des tibialen Bohrkanals die Apophyse der proximalen Tibia oder der ventrale Abschnitt der proximalen Wachstumsfuge der Tibia verletzt worden sein und dadurch $\mathrm{zu}$ einem frühzeitigen Fugenschluss in diesem Bereich geführt haben (Kocher et al. 2002; Seil und Robert 2005). Auch wäre durch das fugennahe, tangentiale Bohren eine Hitzeschädigung der Apophyse der Tuberositas tibiae möglich, welche einen vorzeitigen Wachstumsstopp als Folge haben könnte (Seil 2002). Die Apophyse der Tuberositas tibiae kann auch bei der Entnahme des Patellasehnentransplantats verletzt werden (Woods und O'Connor 2004). Eine weitere Möglichkeit der Ausbildung eines Genu recurvatum ist insbesondere bei den fugenschonenden Techniken denkbar. Beim ventralen Herausziehen des Transplantates aus dem Kniegelenk proximal der tibialen Wachstumsfuge und seiner Fixierung distal der Fuge, könnte das Wachstum der Fuge ventralseitig durch den Zug des Transplantats blockiert werden. In allen diesen Fällen würde ein weiteres Wachstum im dorsalen Bereich der tibialen Wachstumsfuge eine Achsabweichung im Sinne eines Genu recurvatum bedingen.

\subsubsection{Mögliche Ursachen für ein Genu valgum}

Viermal wurde in den ausgewerteten Publikationen die Entwicklung eines Genu valgum berichtet. Von Koman et al. wurde als Ursache für die in dieser Studie beschriebene Achsenabweichung von 14 Grad eine Transfix-Schraube angegeben, mit der das Transplantat femoral befestigt wurde. Die Schraube kreuzte den lateralen Bereich der femoralen Epiphysenfuge und hinderte diese am Wachstum (Koman und Sanders 1999). Da der Ursprung des VKB an der Innenseite der lateralen Femurkondyle liegt, wird auch der Bohrkanal für einen anatomischen Kreuzbandersatz durch den lateralen Teil der femoralen Wachstumsfuge gelegt. Kommt es in dieser Position zu einer Wachstumshemmung der Fuge, zum Beispiel durch ein Implantat zur Transplantatbefestigung, den Knochenblock eines Patellasehnentransplantats oder eine sich ausbildende Knochenbrücke, so bedingt das mediale Weiterwachsen der Fuge eine Achsenabweichung mit resultierendem Genu valgum (Koman und Sanders 1999).

Die im Rahmen der Multicenterstudie beobachtete Valgusabweichung von acht Grad im Vergleich zur nicht operierten Seite wurde vom nachuntersuchenden Chirurgen auf eine 
Verletzung des Ranvierschen Schnürrings zurückgeführt, die durch einen zu weit dorsal gebohrten femoralen Knochenkanal bedingt war.

Auch aus einem Tierversuch (Seil et al. 2008) ist bekannt, dass ein $\mathrm{zu}$ randständiges transphysäres Bohren des femoralen Kanals mit einer Verletzung des Ranvierschen Schnürrings einhergehen kann, die zu einem frühzeitigen Verschluss des lateralen Abschnitts der femoralen Wachstumsfuge und dadurch zu einem Genu valgum führen kann (Seil et al. 2008). Aber auch bei fugenschonenden Operationstechniken kann es zu einem Genu valgum kommen (Lipscomb und Anderson 1986; Seil und Robert 2005). Denkbar wäre eine Wachstumshemmung der lateralen Seite der distalen Wachstumsfuge des Femurs durch ein Befestigen des Transplantats auf dem lateralen Rand der Fuge oder auch hier eine Beschädigung des Ranvierschen Schnürrings (Bales et al. 2004; Seil 2002).

\subsubsection{Mögliche Ursachen für ein Genu varum}

Die Entstehung eines Genu varum wurde in der Multicenterstudie nicht beobachtet und in der analysierten Literatur nur einmal beschrieben (Seil und Robert 2005). Die Achsenabweichung hatte sich im Bereich der tibialen Wachstumsfuge entwickelt, nachdem der Patient eine Kreuzbandplastik mit fugenschonender Operationstechnik erhalten hatte. Das Patellasehnentransplantat war auf der tibialen Epiphyse in einer $1 \mathrm{~cm}$ tiefen knöchernen Nut fixiert worden. Eine Erklärung für diese Komplikation wurde von den Autoren nicht gegeben. Eine prinzipiell denkbare Ursache für die Entstehung eines Genu varum wäre eine Wachstumshemmung des medialen bzw. eine Stimulation des lateralen Anteils der proximalen Wachstumsfuge der Tibia oder der distalen Wachstumsfuge des Femurs.

\subsubsection{Bedeutung für die Therapie}

Auf Grundlage der genannten möglichen Ursachen für die verschiedenen Wachstumsstörungen lassen sich einige Empfehlungen für die Therapie der VKB-Ruptur im Wachstumsalter aussprechen, deren Berücksichtigung das Risiko für eine Wachstumsstörung verringern kann.

- Der operierende Chirurg sollte sehr gute Kenntnisse über die spezifischen anatomischen Gegebenheiten des Kniegelenks bei Patienten im Wachstumsalter besitzen (Seil und Robert 2005). 
- Das Weichteiltransplantat sollte die Wachstumsfuge im Bohrkanal überbrücken, da ein leerer Bohrkanal die Ausbildung einer Knochenbrücke über die Fuge begünstigen kann (Behr et al. 2001; Seil et al. 2008).

- Die Platzierung von Knochenblöcken oder Implantatmaterialien auf Höhe der Wachstumsfuge sollte vermieden werden (Koman und Sanders 1999).

- Um ein Genu recurvatum als Folge einer Apophysenverletzung der Tuberositas tibiae zu vermeiden, sollte ein ausreichender Abstand des tibialen Bohrkanals zur Apophyse eingehalten werden (Kocher et al. 2002; Shea et al. 2003).

- Der tibiale Bohrkanal sollte möglichst nahe dem Zentrum der Fuge liegen. Der femorale Bohrkanal sollte so platziert werden, dass dorsal noch eine Knochenbrücke bestehen bleibt, da besonders durch randständige Verletzung der femoralen Epiphysenfuge ein Genu valgum induziert werden kann (Ford und Key 1956; Shea et al. 2007).

- Auch wenn die Wachstumsfuge nicht durchbohrt wird, muss darauf geachtet werden, dass die Fuge, bzw. der perichondrale Ring, nicht von außen durch Fixierungsmaterial, die Präparation oder andere Manipulationen beschädigt wird (Kocher et al. 2002). Vor allem femoral ist dies von besonderer Bedeutung, da die over-the-top-Position, in der das Band häufig bei fugenschonenden Verfahren befestigt wird, auf Höhe der distalen femoralen Wachstumsfuge liegt (Bales et al. 2004).

- Die Durchmesser der Bohrkanäle durch die Wachstumsfugen sollten möglichst klein gewählt werden (Kocher et al. 2002; Seil und Robert 2005).

- Die Vorspannung des Transplantats sollte nicht extrem hoch sein, um eine Epiphysiodese zu vermeiden (Edwards et al. 2001).

- Um eine Hitzenekrose der Wachstumsfuge zu verhindern, wird empfohlen, die Knochenkanäle mit einer geringen Drehzahl des Bohrers zu bohren (Seil 2002).

- Einige Autoren empfehlen, nicht ein Patellasehnentransplantat mit Knochenblöcken, sondern ein Hamstringtransplantat zu verwenden, da die Knochenblöcke des Patellasehnentransplantats eine Fugenverknöcherung bewirken könnten und bei der Entnahme des Transplantats die proximale Wachstumsfuge der Tibia verletzt werden könnte (Kocher et al. 2002; Mohtadi und Grant 2006; Woods und O'Connor 2004). Diese Befürchtung wird jedoch von der hier vorliegenden Arbeit nicht unterstützt. 
- Zur Einschätzung des Risikos für eine Wachstumsstörung ist es von Vorteil, wenn dem Operateur das noch verbleibende Wachstumspotential des Patienten bekannt ist (Aronowitz et al. 2000). Dieses kann mithilfe eines Röntgenbildes der Hand, dem Tanner-Stadium oder mit der Körpergröße und Wachstumscharakteristik der Eltern abgeschätzt werden (Aronowitz et al. 2000; Fehnel und Johnson 2000).

Auch bei Berücksichtigung aller technischen Aspekte kann es in Einzelfällen zu Wachstumsstörungen kommen. Daher sollten nach jeder vorderen Kreuzbandplastik regelmäßige postoperative Kontrollen bis zum Abschluss des Wachstums erfolgen (Seil und Robert 2005).

\subsection{Können bei Patienten bis zu zwölf Jahren mit einer vorderen Kreuzbandplastik zufriedenstellende Ergebnisse erreicht werden? Erzielen diese Patienten ein schlechteres Therapieergebnis als ältere Patienten mit offenen Wachstumsfugen?}

Mit einer VKB-Plastik lassen sich bei Patienten bis zu zwölf Jahren grundsätzlich zufriedenstellende Therapieergebnisse erreichen. Das erhebliche Risiko einer Wachstumsstörung muss aber unbedingt in die Therapieüberlegungen mit eingeschossen und weitestmöglich minimiert werden.

Die 25 im Rahmen der Multicenterstudie nachuntersuchten Patienten erreichten einen durchschnittlichen Lysholm-Score von 93,0 (42 bis 100) Punkten, dies entspricht einem guten Ergebnis. Ein Patient schnitt mit 42 Punkten schlecht, ein anderer mit 70 Punkten mäßig ab. Die anderen Patienten hatten entweder gute oder sehr gute Punktwerte. Für zwei Patienten konnte der Lysholm-Score aufgrund unvollständiger Angaben nicht berechnet werden. Bei der Nachuntersuchung des Kniegelenkes nach IKDC wurden die operierten Knie siebenmal als normal (A), 13-mal als fast normal (B), einmal als abnormal (C) und einmal als deutlich abnormal (D) eingestuft. Bei drei Patienten wurde diese Untersuchung nicht durchgeführt. Bei einem der 25 Patienten (4 Prozent) trat eine Reruptur auf. Die Rate an Beinlängendifferenzen oder Achsabweichungen war mit einem Auftreten bei sechs von 25 (24 Prozent) hoch. Der Patient mit dem schlechten Lysholm-Score und dem deutlich abnormalen IKDC hatte direkt vor der Nachuntersuchung ein erneutes Distorsionstrauma erlitten und sich dabei die Reruptur zugezogen.

In der publizierten Literatur werden individuelle Ergebnisse der Patienten von bis zu zwölf Jahren nur selten beschrieben. So war bei 30 der insgesamt 59 Artikel, die über Patienten mit offenen Wachstumsfugen berichteten, entweder gar nicht erkennbar ob überhaupt Patienten 
mit maximal zwölf Jahre behandelt wurden oder die Ergebnisse wurden nur in Form von Mittelwerten für junge und ältere Patienten mit offenen Wachstumsfugen zusammen beschrieben. 37 mit Kreuzbandplastik versorgte Patienten und einem Alter von maximal zwölf Jahren konnten separat ausgewertet werden. Für sie wurden bei einem durchschnittlichen Lysholm-Score von 95,6 (84-100) Punkten ausschließlich gute und sehr gute klinische Ergebnisse berichtet. Eine Reruptur trat keinmal auf, eine Beinlängendifferenz oder Achsenabweichung bei zwei von 32 (6,3 Prozent), für fünf wurden keine eindeutigen Angaben gemacht.

Dass die Rate an Beinlängendifferenzen oder Achsenabweichungen in der Multicenterstudie deutlich höher lag als bei den bis zu zwölfjährigen Patienten in der Literaturanalyse, könnte damit zusammen hängen, dass die nachuntersuchenden Chirurgen der Multicenterstudie durch den Nachuntersuchungsbogen dazu angehalten waren, speziell nach Wachstumsstörungen zu suchen. Es ist denkbar, dass diese sonst nicht auffallen, weil sich die betroffenen Patienten häufig beschwerdefrei präsentieren.

Die Therapieergebnisse der bis zu Zwölfjährigen wurden in der vorliegenden Arbeit den Ergebnissen aller Patienten mit offenen Wachstumsfugen gegenübergestellt. Diese Gegenüberstellung wurde dem Vergleich mit Patienten mit einem Alter von mindestens 13 Jahren vorgezogen. Wie oben beschrieben, ist bekannt, dass die überwiegende Mehrzahl der Patienten mit einer ligamentären Kreuzbandruptur bei offenen Wachstumsfugen älter als zwölf Jahre alt ist. In den Publikationen wird das Alter der einzelnen Patienten jedoch nur selten angegeben, so dass die Unterscheidung in eine Gruppe von mindestens 13-jährigen schwierig ist.

Das klinische Ergebnis war bei der Gruppe aller 769 überwiegend älteren Patienten mit offenen Wachstumsfugen mit einem durchschnittlichen Lysholm-Score von 94,4 (62-100) Punkten ähnlich den publizierten Ergebnissen der bis zu zwölfjährigen. Die Rerupturrate war mit 30 von 769 (3,9 Prozent) etwas schlechter, die Rate an beschriebenen Beinlängendifferenzen und Achsenabweichungen mit 29 von 769 (3,8 Prozent) niedriger.

Prinzipiell besteht eine mögliche Ursache für eine höhere Fehlwachstumsrate bei den bis zu zwölf Jahren alten Patienten darin, dass die Wachstumsfugen aller dieser Patienten zum Zeitpunkt der Operation noch weit geöffnet sind und deren Wachstumspotential noch größer ist als das der älteren Patienten (Seil 2002). Ein Sistieren des Längenwachstums ist bei Mädchen etwa mit 14 bis 15, bei Jungen mit 16 bis 17 Jahren zu erwarten (Bales et al. 2004; Seil und Robert 2005). Auch wenn alle 769 mit Kreuzbandplastik versorgten Patienten zum 
Zeitpunkt der Operation noch offene Wachstumsfugen besaßen, so stand doch bei vielen Patienten die natürliche Verknöcherung der Fuge und ein Sistieren des Längenwachstums kurz bevor. Es ist davon auszugehen, dass eine Irritation der Wachstumsfuge bei den Patienten unter 13 Jahren eine größere Auswirkung auf die Beinlänge und Beinachse hat als bei älteren Patienten.

Dass sich auch für junge Patienten im Alter von bis zu zwölf Jahren mit einer Kreuzbandplastik gute und sehr gute Ergebnisse erzielen lassen, wird auch durch eine Studie von Kocher et al. bestätigt, in welcher 44 Patienten mit einem Durchschnittsalter von 10,3 (3,6 bis 14) Jahren mit einer vorderen Kreuzbandplastik in fugenschonender Technik versorgt wurden. Nach durchschnittlich 5,3 (2,0 bis 15,1) Jahren wurden die Patienten nachuntersucht und wiesen gute bis sehr gute Ergebnisse und keine Wachstumsstörungen auf. Sie waren innerhalb dieser Zeit durchschnittlich 21,5 (9,5 bis 118,5) cm gewachsen (Kocher et al. 2005).

Die Gefahr, durch die Operation eine irreversible Beinlängendifferenz oder Achsenabweichung zu verursachen, ist jedoch bei Patienten bis zu zwölf Jahren vermutlich größer als bei denen, die sich kurz vor dem Ende ihres Längenwachstums befinden.

\subsection{Ist bei Patienten bis zu zwölf Jahren eine fugenschonende Technik notwendig, oder können die Transplantatkanäle fugenkreuzend angelegt werden?}

Die Frage, ob Patienten mit offenen Wachstumsfugen mit einer VKB-Insuffizienz fugenkreuzend operiert werden können, oder ob eine fugenschonende Operationstechnik nötig ist, ist eine der zentralen Fragen in der kindlichen und jugendlichen Kreuzbandchirurgie (Schachter und Rokito 2007; Sobau und Ellermann 2004). Viele Autoren verweisen auf das Risiko einer iatrogenen Wachstumsstörung durch die Verletzung der Wachstumsfugen bei den fugenkreuzenden Verfahren (Cohen et al. 2009; Lukas et al. 2007; Seil 2002; Vaquero et al. 2005). Das Risiko des Auftretens dieser Komplikationen hat zur Anwendung einer Vielzahl verschiedener wachstumsfugenschonender Operationstechniken geführt (Kocher et al. 2005; Robert und Bonnard 1999; Seil und Robert 2005)

In den beteiligten Krankenhäusern der Multicenterstudie wurden zwei Patienten fugenschonend operiert. Der Lysholm-Score wurde bei der Nachuntersuchung nicht bestimmt, bei der Untersuchung nach IKDC wurde das Knie eines Patienten als abnormal (C), das des anderen als fast normal (B) eingestuft. Eine Reruptur trat nicht auf, aber beide Patienten (100 Prozent) entwickelten eine Beinlängendifferenz. Bei 23 weiteren Patienten wurde eine 
fugenkreuzende Operationstechnik angewandt. Der Lysholm-Score betrug bei der Nachuntersuchung 93,0 (42 bis 100) Punkte, der IKDC war bei sieben Patienten normal (A), bei zwölf fast normal (B), bei einem deutlich abnormal (D), dreimal wurde er nicht bestimmt. Eine Reruptur trat bei einem der Patienten (4,3 Prozent) auf, eine Achsenabweichung oder Beinlängendifferenz wurde bei vier von ihnen (17,4 Prozent) festgestellt.

Die publizierte Literatur zu Patienten bis zu zwölf Jahren ist spärlich. Beschrieben werden 18 Patienten, welche mit verschiedenen fugenschonenden Kreuzbandersatztechniken behandelt wurden. Das klinische Ergebnis ist mit einem Lysholm-Score von durchschnittlich 96,8 (93 bis 100) Punkten sehr gut. Keinmal kam es zu einer Reruptur, jedoch entwickelte ein Patient (5,6 Prozent) eine Beinlängendifferenz und ein anderer (5,6 Prozent) ein Genu recurvatum. Auch die beschriebenen zwölf fugenkreuzend operierten Patienten, erzielten durchschnittlich ein sehr gutes klinisches Ergebnis bei einem Lysholm-Score von 96 (89 bis 100) Punkten. Bei keinem dieser Patienten kam es zu einer Reruptur, Beinlängendifferenz oder Achsenabweichung.

Die Ergebnisse der Multicenterstudie und der Literaturanalyse sprechen somit tendenziell nicht für die Favorisierung von fugenschonenden Operationsmethoden gegenüber fugenkreuzenden Techniken bei Patienten bis zwölf Jahren. Das Risiko einer iatrogenen Wachstumsstörung scheint durch fugenschonende Techniken nicht wesentlich reduziert werden zu können.

Aufgrund der wenigen publizierten Daten für diese Altersgruppe wurde zur Klärung der Frage, ob fugenschonende Techniken notwendig sind, oder die Transplantatkanäle fugenkreuzend angelegt werden können, ergänzend die Literatur für alle Patienten im Wachstumsalter analysiert.

\subsection{Ist eine fugenschonende Operationstechnik bei der vorderen Kreuzbandplastik bei offenen Wachstumsfugen generell nötig oder kann fugenkreuzend operiert werden?}

643 Patienten mit offenen Wachstumsfugen konnten in der systematischen Literaturanalyse ausgewertet werden. 154 Patienten wurden sowohl femoral als auch tibial fugenschonend operiert. Zum Zeitpunkt der Nachuntersuchung hatten sie einen Lysholm-Score von 95,3 (74 bis 100) Punkten, bei dreien (1,9 Prozent) war eine Reruptur, bei 17 (11 Prozent) eine Beinlängendifferenz oder Achsenabweichung des operierten Beines aufgetreten. Unter den fugenschonenden Operationsverfahren wurde eine große Zahl verschiedener Operationstechniken zusammengefasst, welche alle auf eine Transplantatführung durch die 
Wachstumsfugen verzichteten. Diese Techniken einzeln auszuwerten war aufgrund der Vielzahl und der geringen Behandlungszahlen der einzelnen Techniken nicht möglich.

489 Patienten wurden femoral als auch tibial fugenkreuzend operiert. Der Lysholm-Score bei der Nachuntersuchung betrug 94,4 (89 bis 100) Punkte, eine Reruptur wurde bei 24 (4,9 Prozent) der Patienten, eine Beinlängendifferenz oder Achsabweichung bei sieben $(1,4$ Prozent) beobachtet.

Bei der Interpretation der Ergebnisse ist zu berücksichtigen, dass das Durchschnittsalter der Gruppe mit fugenkreuzenden Verfahren mit 13.8 (9 bis 17) Jahren deutlich über demjenigen der Gruppe fugenschonender Techniken mit 11.9 Jahren (2 bis 17) liegt. Da vermutlich bei einem Teil vor allem der Patienten mit fugenkreuzenden Verfahren der natürliche Schluss der Epiphysenfugen zum Zeitpunkt der Operation schon kurz bevor stand, hatte bei diesen Patienten eine Fugenirritation möglicher Weise weniger Auswirkungen auf das Wachstum als bei den jüngeren Patienten mit noch offenen Wachstumsfugen.

Trotz der häufig geäußerten Gefahr eines Fehlwachstums durch eine Fugendurchbohrung (Cohen et al. 2009; Lukas et al. 2007; Seil 2002; Vaquero et al. 2005) werden die beidseits fugenkreuzenden Verfahren bei Patienten mit offenen Wachstumsfugen deutlich häufiger angewendet als die fugenschonenden Techniken. Zu diesem Ergebnis kamen bereits 2002 auch Kocher et al. in ihrer internationalen Befragung von Kreuzbandspezialisten.

In der Literatur wird als Nachteil der fugenschonenden Technik die Schwierigkeit genannt, das Transplantat in die anatomisch korrekte Lage $\mathrm{zu}$ bringen und wieder eine normale Physiologie des Kniegelenkes herzustellen (Dorizas und Stanitski 2003; Vaquero et al. 2005; Woods und O'Connor 2004). Die Gefahr eines Fehlwachstums wird als Nachteil der fugenkreuzenden Techniken genannt (Vaquero et al. 2005, Schachter und Rokito 2007). Die Ergebnisse der Multicenterstudie und der systematischen Literaturanalyse sprechen aber dafür, dass bei Patienten mit offenen Wachstumsfugen auch durch fugenschonende Techniken Wachstumsstörungen verursacht werden können. In der Literaturanalyse lag deren Rate an Beinlängendifferenzen bzw. Achsenabweichungen sogar weit höher, als bei den fugenkreuzenden Verfahren. Auch wenn die Wachstumsfugen nicht durchbohrt werden, sollte beim Kreuzbandersatz daher immer an das Risiko einer Wachstumsstörung gedacht werden. Es ist sogar in Frage zu stellen, ob eine Durchbohrung der Wachstumsfugen überhaupt ein höheres Risiko für eine Wachstumsstörung bedeutet als ein Verfahren, bei dem die Fugen zwar nicht durchbohrt, aber eventuell auf andere Art irritiert werden. 
Mehrere Autoren machen den Vorschlag, zur Überbrückung der Zeit bis zu einem Wachstumsfugenverschluss, in einer ersten Operation fugenschonende Techniken anzuwenden, um dann später in einer zweiten Operation fugenkreuzend zu rekonstruieren (Dorizas und Stanitski 2003; Micheli et al. 1999; Schachter und Rokito 2007). Die hohe Zahl der in der vorliegenden Arbeit detektierten Beinlängendifferenzen oder Achsenabweichungen nach fugenschonender Rekonstruktion stellt dieses Vorgehen jedoch grundsätzlich in Frage.

\subsection{Welches Transplantat kann für die Kreuzbandplastik bei Patienten bis zu zwölf Jahren und bei Patienten im Wachstumsalter (altersunabhängig) empfohlen werden?}

In der Literatur mit Abstand am häufigsten beschrieben ist die Verwendung von autologen Transplantaten der Semitendinosus-Grazilis-Sehne und der Patellasehne (Kocher et al. 2002). Seltener kommen auch autologe Quadrizepssehnentransplantate (Gebhard et al. 2006; Lo et al. 1997) und Transplantate der Fascia lata (Gebhard et al. 2006; Kocher et al. 2005) zur Anwendung. Patellasehnentransplantate zeichnen sich durch eine von Anfang an hohe Reißfestigkeit aus (Noyes et al. 1984). Da die Patellasehne zusammen mit angrenzenden Knochenblöcken implantiert werden kann, erfolgt eine schnelle Einheilung des Transplantats (Aronowitz et al. 2000). Nachteil sind die vergleichsweise häufigen Komplikationen an der Entnahmestelle, vor allem Schmerzen und einer Schwäche des Musculus quadriceps (Aronowitz et al. 2000). Einige Autoren geben zusätzlich zu bedenken, dass die transplantierten Knochenblöcke des Patellasehnentransplantats eine Fugenverknöcherung bedingen könnten und dass bei der Entnahme des Transplantats die proximale tibiale Wachstumsfuge verletzt werden kann. Beides könnte Wachstumsstörungen bedingen (Kocher et al. 2002; Mohtadi und Grant 2006; Woods und O'Connor 2004). Im Vergleich zum Patellasehnentransplantat kommt es bei Verwendung der Semitendinosus-Grazilis-Sehne seltener zu Entnahmekomplikationen. Der benötigte Zugang zum Entnahmegebiet ist kleiner. Nachteil dieses Transplantats ist eine häufigere postoperative anteriore Laxizität des Kniegelenks (Aronowitz et al. 2000). Die Semitendinosus-Grazilis-Sehne kann entweder vollständig entnommen werden oder aber mit ihrem natürlichen Ansatz an der Tibia belassen und von hier aus durch das Gelenk gezogen werden (Kocher et al. 2002).

Von den autologen Transplantaten müssen allogene unterschieden werden. Unter ihnen werden am häufigsten die Achillessehne (Andrews et al. 1994; Aronowitz et al. 2000; Sankar et al. 2006) und die Patellasehne (Fuchs et al. 2002; Vaquero et al. 2005) verwendet. Vorteil der allogenen Transplantate gegenüber der autologen ist, dass die Operationsdauer kürzer ist, 
da die Transplantatentnahme entfällt und das Transplantat direkt eingebracht werden kann. Außerdem ist die Operation nicht wie bei den autologen Transplantaten mit möglichen Schmerzen und Komplikationen an der Entnahmestelle verbunden. Nachteil ist jedoch das Risiko einer Krankheitsübertragung mit dem Transplantat, eine mögliche Autoimmunreaktion des operierten Patienten und eine verlangsamte Einheilung des Transplantats (Aronowitz et al. 2000; Vaquero et al. 2005). Diese Nachteile und die Sorge, das Transplantat könne durch die Sterilisierung in seinen mechanischen Eigenschaften beeinträchtigt werden, haben dazu beigetragen, dass allogene Transplantate nur selten verwendet werden (Dorizas und Stanitski 2003).

In einem Artikel von Tallay et al. (2008) wird außerdem von einem elf Jahre alten Patienten berichtet, welcher nach einer VKB-Ruptur die Semitendinosus-Grazilis-Sehne der eigenen Mutter als Kreuzbandersatz implantiert bekam. Das Operationsergebnis war gut.

Eine vor noch wenigen Jahren bestehende Unsicherheit galt dem Verhalten des eingebrachten Transplantats über die Wachstumsphase hinweg. Da das Transplantat in den meisten Fällen proximal der distalen femoralen und distal der proximalen tibialen Wachstumsfuge befestigt wird, bewegen sich diese Ankerpunkte mit dem Wachstum der unteren Extremität voneinander fort. Aktuelle Studien konnten zeigen, dass das Transplantat in Länge und Durchmesser mit dem Knie mitwächst (Liddle et al. 2008).

Im Rahmen der Multicenterstudie wurde bei zwei der 25 Patienten ein autologes Patellasehnentransplantat mit femoralem Knochenblock verwendet. Für diese Patienten wurde bei der Nachuntersuchung kein Lysholm-Score erhoben, der IKDC war einmal abnormal (C) und einmal fast normal (B). Es kam zu keiner Reruptur, beide (100 Prozent) wiesen bei der Nachuntersuchung eine Beinlängendifferenz auf. Die restlichen 23 Patienten wurden mit einem Hamstringtransplantat versorgt, ihr Lysholm-Score betrug bei der Nachuntersuchung 93,0 (42 bis 100) Punkte, der IKDC fiel bei sieben Patienten normal aus (A), bei zwölf fast normal (B) und bei einem deutlich abnormal (D). Eine Reruptur war einmal (4,3 Prozent) aufgetreten eine Achsabweichung oder Beinlängendifferenz bei vier der 23 Patienten (17,4 Prozent).

Von den 37 publizierten Fällen von Patienten bis zwölf Jahren wurden 14 mit Hamstring, zwölf mit Patellasehne, sechs mit Fascia lata und fünf mit einem Allografttransplantat versorgt. Die mit Hamstringtransplantat versorgten Patienten erreichten im Durchschnitt einen Lysholm-Score von 89,3 (84 bis 94) Punkten und damit „nur“ ein gutes klinisches Ergebnis. Die anderen Transplantatgruppen erzielten jeweils sehr gute klinische Ergebnisse in Form der 
Lysholm-Scores. Bei keinem der 37 Patienten wurde eine Reruptur beobachtet. Bei zwei (33,3 Prozent) der sechs Patienten, die einen VKB-Ersatz mit einem Fascia-lata-Transplantat erhalten hatten, wurde eine Wachstumsstörung festgestellt, bei einem Patient wurde bei der Nachuntersuchung eine Beinlängendifferenz, bei dem anderen ein Genu recurvatum verzeichnet. Jedoch sind die Patientenzahlen dieser verschiedenen Gruppen zu klein, um von diesen Ergebnissen allgemeingültige Aussagen abzuleiten. Daher wurden die publizierten Operationsergebnisse zusätzlich altersunabhängig für Patienten mit offenen Wachstumsfugen ausgewertet.

Die häufigste Anwendung fand mit 359 Patienten die Semitendinosus-Grazilis-Sehne, ihr folgte die Patellasehne mit 200 Patienten. Im Lysholm-Score unterschieden sich die Ergebnisse aller verwendeten Transplantate nicht wesentlich. Bezüglich der Rerupturrate lag die Gruppe der Quadrizepssehnentransplantate mit drei von 14 (21,4 Prozent) deutlich über allen anderen Transplantatgruppen (Gebhard et al. 2006). Das beste Ergebnis in Bezug auf die Rerupturrate hatte die Gruppe der Patellasehnentransplantate mit fünf von 200 (2,5 Prozent) (Edwards und Grana 2001; McCarroll et al. 1994; Shelbourne et al. 2004). Die Rate an Wachstumsstörungen war mit sechs von 79 (7,6 Prozent) bei den mit Fascia-lata-Transplantat versorgten Patienten am höchsten (Micheli et al. 1999; Nakhostine et al. 1995). Die Patienten dieser Gruppe hatten auch ein sehr niedriges Durchschnittsalter von 10,3 Jahren. Beachtlich ist, dass die Fehlwachstumsrate der mit einem Patellasehnentransplantat versorgten Patienten mit fünf von 200 (2,5 Prozent) deutlich unter der Rate der Patienten mit einer Semitendinosus-Grazilis-Sehne lag. Bei letzteren kam es in 16 von 359 (4,5 Prozent) der Fälle zu einer Beinlängendifferenz bzw. Achsenabweichung. Diese Ergebnisse widersprechen der Empfehlung mehrerer Autoren, Patellasehnentransplantate bei Patienten mit offenen Wachstumsfugen aufgrund des erhöhten Risikos einer Wachstumsstörung zu vermeiden (Kocher et al. 2002; Mohtadi und Grant 2006; Woods und O'Connor 2004). Wichtig ist aber eine Überbrückung der Wachstumsfugen mit dem sehnigen Anteil des Patellasehnentransplantats (Seil und Robert 2005), um die Bildung von Knochenbrücken über die Fugen zu verhindern.

Eine klare Empfehlung für ein bestimmtes Transplantat kann auf Grundlage dieser Ergebnisse nicht gegeben werden. Vermutlich hat die Technik der Transplantateinbringung und die Platzierung der Bohrtunnel einen größeren Einfluss auf das Operationsergebnis als die Wahl des Transplantats (Aronowitz et al. 2000; Dorizas und Stanitski 2003). 


\subsection{Der Einfluss des Zugangsweges zur Anlage des femoralen Knochenkanals auf das Auftreten von Beinlängendifferenzen oder Achsenanbweichungen}

Um das Risiko einer Wachstumsstörung zu minimieren, gibt es die Empfehlung, die Wachstumsfugen bei der Bohrung der Knochenkanäle möglichst senkrecht zu perforieren (Sobau und Ellermann 2004). Denn je flacher der Winkel des Bohrkanals zur Fuge ist, desto mehr Volumen des Fugenknorpels wird durch die Bohrung zerstört (Kercher et al. 2009). Für den femoralen Knochenkanal erfordert eine möglichst senkrechte Kanalanlage, dass die Bohrung nicht wie beim Erwachsenen üblich über einen anteromedialen Zugangsweg gebohrt wird, sondern transtibial.

In der Multicenterstudie wurde von 21 anteromedialen Zugangswegen berichtet. Von diesen Patienten entwickelten fünf (23,8 Prozent) eine Beinlängendifferenz oder Achsenabweichung. Zweimal wurde transtibial gebohrt, hier kam es zu keiner Wachstumsstörung. Für zwei Patienten wurde die Kanalanlage nicht beschrieben. Ob dieser Ergebnisunterschied auf die Wahl des Zugangsweges zurückgeführt werden kann, bleibt aufgrund der nur zweifachen Anwendung des transtibialen Zugangsweges offen.

Die systematische Literaturanalyse für Patienten bis zum Alter von zwölf Jahren ergab keine Beinlängendifferenz oder Achsabweichung bei beiden Verfahren. Auch für die gesamte (altersunabhängige) Gruppe der Patienten im Wachstumsalter ergab die systematische Literaturanalyse keine wesentlichen Unterschiede in der Anzahl der Beinlängendifferenzen oder Achsenabweichungen hinsichtlich der verwendeten Technik für die Anlage des femoralen Kanals. Die Daten der Literaturanalyse sprechen somit dafür, dass auch im Hinblick auf eine Minimierung des Risikos für eine Wachstumsstörung, die Anlage des femoralen Kanals über einen anteromedialen Zugang vertretbar ist.

\subsection{Gelenkferne oder gelenknahe Fixierung?}

Ein grundsätzlicher Nachteil einer gelenkfernen Fixierung ist, dass das Transplantat eine große Strecke unfixiert durch den knöchernen Kanal verläuft, bevor es in diesem befestigt ist. Die dynamische Zugbelastung bedingt feine Bewegungen des frei im Knochenkanal verlaufenden Transplantats und erschwert seine Einheilung. Einige Zeit postoperativ kann eine Vergrößerung des Tunneldurchmessers in diesem Bereich beobachtet werden (Hersekli et al. 2004). Fauno und Kaalund (2005) konnten in einer prospektiv randomisierten Studie zeigen, dass diese Tunnelaufweitung bei einer gelenkfernen Fixierung signifikant größer 
ausfällt als bei einer gelenknahen. Dieses Phänomen hatte in deren Studie jedoch keinen Einfluss auf das klinische Ergebnis der Patienten. In der Literatur gibt es die Empfehlung, diesen ungewollten Effekt der Tunnelaufweitung bei gelenkferner Fixierung in Kauf zu nehmen, da das Risiko einer dauerhaften Störung der Wachstumsfuge durch Implantatmaterial und für daraus resultierende Wachstumsstörungen bei gelenknaher Fixierung ungleich höher sei; denn das Implantatmaterial zur Befestigung des Transplantats muss bei gelenknaher Fixierung näher an der Fuge platziert werde (Lukas et al. 2007).

Entgegen dieser Befürchtung zeigte in der systematischen Literaturanalyse die gelenknahe Transplantatfixierung sowohl in der Gruppe der Patienten bis zu zwölf Jahren - keiner von acht (0 Prozent) gegenüber zwei von 18 (11,1 Prozent) bei gelenkferner Fixierung - als auch in der Gruppe aller Patienten mit offenen Wachstumsfugen - einer von 56 (1,8 Prozent) gegenüber 13 von 506 (2,6 Prozent) - keine höhere Rate an späteren Beinlängendifferenzen oder Achsenabweichungen.

Bezüglich des Lysholm-Scores und der Rerupturrate zeigten sowohl die bis zu zwölf Jahren alten Patienten als auch das gesamte Altersspektrum von Patienten mit offenen Wachstumsfugen nur unwesentlich verschiedene Ergebnisse der beiden Techniken.

Trotz räumlicher Nähe des Implantationsmaterials zur Wachstumsfuge zeigte sich die gelenknahe Transplantatbefestigung somit nicht von Nachteil.

\subsection{Naht der Bandstümpfe bei Kreuzbandverletzungen im Wachstumsalter}

Wenige Studien berichten von Patienten im Wachstumsalter deren Kreuzbandruptur mit einer Naht behandelt wurde. Nur für 15 Patienten in sechs Studien wird das Alter angegeben. Sechs Patienten sind zum Zeitpunkt der Operation bis zu zwölf Jahren alt, neun sind mindestens 13 Jahre alt (Bradley et al. 1979; DeLee und Curtis 1983; Engebretsen et al. 1988; Gaudernak 1996; Schaefer et al. 1993). Für keinen Patienten wird das klinische Ergebnis in Form des Lysholm-Scores beschrieben, keiner entwickelte eine Beinlängendifferenz oder Achsenabweichung. Bei zwei (33,3 Prozent) der sechs bis zu zwölfjährigen Patienten und einem (11,1 Prozent) der neun mindestens 13 Jahre alten Patienten kam es zu einer erneuten Ruptur des genähten Bandes (Gaudernak 1996; Schaefer et al. 1993). Mit diesen in der Literatur verfügbaren Daten kann die Frage, ob die Kreuzbandnaht bei Patienten mit einem Alter von bis zu zwölf Jahren erfolgversprechender ist als bei älteren Patienten mit noch offenen Wachstumsfugen, nicht befriedigend beantwortet werden. Jedoch dämpft allein die 
hohe Rate an Rerupturen in der Gruppe der bis zu Zwölfjährigen die Hoffnung, die Heilungstendenz des Bandes in dieser Altersgruppe könnte besser sein als die älterer Jugendlicher oder Erwachsener. Bei diesen sind die Ergebnisse der Kreuzbandnaht ähnlich schlecht wie die einer rein konservativen Therapie (Shea et al. 2003) und gehen meist mit einem starken Rückgang der sportlichen Aktivität der Patienten einher (Aronowitz et al. 2000).

\subsection{Kreuzbandnaht gegenüber Kreuzbandplastik bei offenen Wachstumsfugen}

In der systematischen Literaturanalyse zeigte die Kreuzbandnaht im Vergleich zu der Kreuzbandersatzplastik tendenziell schlechtere Ergebnisse. Das klinische Ergebnis in Form des Lysholm-Scores lag mit durchschnittlich 79,6 Punkten unter dem der Ersatzverfahren mit 94,4 (62 - 100) Punkten. (Für die Kreuzbandnaht war kein minimaler oder maximaler Lysholm-Score zu ermitteln.) Die Rate an Rerupturen war mit 7 von 91 (7,7 Prozent) im Vergleich zu den Kreuzbandplastiken mit 30 von 769 (3,9 Prozent) hoch. Bemerkenswert ist jedoch, dass die Kreuzbandnähte in keinem Falle zu einer Wachstumsstörung führten. Die Kreuzbandplastiken taten dies jedoch in 29 von 769 Fällen (3,8 Prozent). Grund für die schlechten klinischen Ergebnisse der Kreuzbandnähte im Wachstumsalter waren vor allem die persistierende Instabilität der Kniegelenke (Attmanspacher et al. 2003; Engebretsen et al. 1988; Gaudernak 1996; Pressman et al. 1997). Von einer Kreuzbandnaht bei VKB-Rupturen im Wachstumsalter ist daher eher abzuraten (DeLee und Curtis 1983; Engebretsen et al. 1988).

\subsection{Schwächen der Multicenterstudie}

Eine Schwachstelle der Multicenterstudie ist die relativ niedrige Zahl an nachuntersuchten Patienten, was die statistische Aussagekraft begrenzt. Statistische Tests wurden aufgrund der kleinen Patientenzahl als nicht zielführend erachtet. Da es sich um eine retrospektive Studie handelt, war eine Standardisierung der präoperativen Datenerfassung nicht möglich. Es gibt weitere Faktoren die die Vergleichbarkeit der Behandlungsergebnisse verschlechtern: So wurden die nachuntersuchten Patienten sowohl von verschiedenen Chirurgen operiert, als auch von verschiedenen Ärzten nachuntersucht. Auch die Operationstechniken unterschieden sich in mehreren Aspekten. Die Nachbehandlung der Patienten war nicht einheitlich geregelt. Auch wurden zur Beurteilung der Behandlungsergebnisse verschiedene bildgebende 
Verfahren benutzt, ein MRT und/oder Röntgenbild wurde nur von einem Teil der Untersucher erstellt. Da keine vergleichbaren prä- und postoperativen Ganzbeinaufnahmen im Seitenvergleich herangezogen wurden, konnte eine Beinlängendifferenz oder Achsenabweichung nicht hoch exakt quantifiziert werden. Außerdem unterschieden sich die Patienten bezüglich der zum Zeitpunkt der Operation bestehenden Begleitverletzungen des Kniegelenkes, welche ebenso einen Einfluss auf die Ergebnisse der Therapie haben könnten. Es ist davon auszugehen, dass bei einem Teil der Patienten zum Zeitpunkt der Nachuntersuchung das Wachstum noch nicht abgeschlossen war. Eine radiologische Kontrolle, ob die Wachstumsfuge schon verschlossen oder noch offen war, wurde nicht durchgeführt. Daher ist nicht auszuschließen, dass es im weiteren Verlauf noch zu Komplikationen in Form eines Fehlwachstums kommen kann.

\subsection{Schwächen der Literaturanalyse}

Die vorliegende Literaturanalyse wurde systematisch und gründlich durchgeführt, dennoch müssen einige Schwachstellen erwähnt und diskutiert werden.

Alle herangezogenen Studien umfassen aufgrund der Seltenheit des Krankheitsbildes kleine Patientenzahlen. Die Evidenz der ausgewerteten Studien ist niedrig, keine der 59 Studien besitzt einen Evidenzlevel von eins oder zwei. Bei der Mehrheit der Studien handelt es sich um Fallserien. $\mathrm{Zu}$ diesem Ergebnis kamen auch Mohtadi und Grant (2006) in ihrer Literaturanalyse. Die Evidenz der Ergebnisse der vorliegenden systematischen Literaturanalyse ist somit ebenso begrenzt, da sie direkt von der Evidenz der Primärliteratur abhängt.

Es ist denkbar, dass eine Publikations-Bias zu Gunsten von Patienten mit einem guten operativen Ergebnis besteht, da mehrere Autoren eine neue Operationsmethode vorstellen und dieses Vorgehen mit guten Ergebnissen der von ihnen behandelten Patienten unterstreichen.

Andererseits wurden auch Einzelfallberichte ausgewertet, die teilweise vermutlich besonders komplikationsreiche Verläufe beschreiben (Koman und Sanders 1999). Dies wurde bewusst in Kauf genommen, um ein möglichst vollständiges Bild der verschiedenen Komplikationen sowie deren möglichen Ursachen zu erhalten.

Die Interpretation der hier dargestellten Ergebnisse wird auch dadurch erschwert, dass zur Berechnung mancher Werte, Patienten zu Gruppen zusammengefasst wurden, die bezüglich des jeweils untersuchten Merkmals zwar identisch waren, sich aber in anderen Merkmalen voneinander unterschieden. So ist anzunehmen, dass die verschieden schweren 
Begleitverletzungen, die unterschiedlichen Nachbehandlungen, die verschieden langen Intervalle zwischen Unfall und Operation und andere Faktoren ebenfalls einen Einfluss auf das Therapieergebnis hatten. Diese Faktoren wurden aber bei den Auswertungen und der Beurteilung der Behandlungsergebnisse nicht berücksichtigt.

In der präsentierten Literaturanalyse wurden unter der Gruppe der fugenschonenden Operationsverfahren all jene Kreuzbandersatzverfahren zusammengefasst, bei denen die Wachstumsfugen nicht perforiert wurden. Darunter fallen sowohl extraartikuläre als auch intraartikuläre Verfahren.

Die Vielzahl an diagnostischen Tests und klinischen Scores, mit denen das Behandlungsergebnis von den verschiedenen Autoren evaluiert wurde, erschwert den Vergleich zwischen den Studien und erschwert es einen gemeinsamen Maßstab zu finden, anhand dessen alle operierten Patienten miteinander verglichen werden können. Als am häufigsten verwendeter Maßstab hat sich hier der Lysholm-Score erwiesen, er wurde daher in der Literaturanalyse als Parameter für das klinische Ergebnis verwendet. Trotzdem wurde er in einigen Publikationen nicht angegeben, welche dadurch für die Berechnung der klinischen Ergebnisse nicht zur Verfügung standen.

Eine Metaanalyse der Publikationen war aufgrund deren Heterogenität nicht möglich. Für exaktere Aussagen und Empfehlungen müsste die statistische Signifikanz durch eine Regressionsanalyse berechnet werden, hierfür sind jedoch die Fallzahlen bei den meisten untersuchten Aspekten zu gering.

\subsection{Anregungen für zukünftige Studien}

Wie oben beschrieben präsentieren die aktuell publizierten Studien zur operativen Versorgung der VKB-Ruptur im Wachstumsalter kleine Patientenzahlen. Meist handelt es sich um Fallstudien ohne Kontrollgruppen. Zur abschließenden Beantwortung der in dieser Arbeit vorgestellten Fragenfelder wäre es sinnvoll, wenn Studien mit einer höheren Evidenz durchgeführt werden würden.

In Anbetracht der Tatsache, dass der vordere Kreuzbandersatz mehrere Jahrzehnte funktionsfähig bleiben soll und dass eine nicht exakt anatomische Positionierung des Bandes die Belastungsverhältnisse innerhalb des Kniegelenkes dauerhaft verändern kann, sind zukünftige Studien zur Beurteilung der Langzeitergebnisse notwendig (Shea et al. 2003). 
Außerdem mangelt es noch an Untersuchungen zum besten Operationszeitpunkt und zur

Optimierung der Einheilung des Transplantats (Lukas et al. 2007; Schachter und Rokito 2007). 


\section{Zusammenfassung}

Für die Versorgung intraligamentärer vorderer Kreuzbandrupturen bei Patienten im Wachstumsalter wurde - aufgrund der Sorge vor dauerhaften Wachstumsstörungen durch eine Schädigung der Wachstumsfugen - eine Vielzahl verschiedener operativer Techniken entwickelt. Noch hat sich keines der praktizierten Verfahren eindeutig etablieren können. Dies gilt ganz besonders für Patienten, welche noch weit vom Abschluss des Wachstums entfernt sind.

Ziel dieser Arbeit war es, die angewandten Operationsverfahren hinsichtlich ihrer klinischen Ergebnisse und Risiken zu untersuchen. Dies sollte insbesondere für Patienten bis zu einem Alter von zwölf Jahren und vergleichend für alle Patienten im Wachstumsalter geschehen.

In einer retrospektiven nationalen Multicenterstudie wurden Patienten, die eine vordere Kreuzbandoperation erhalten hatten, anhand eines standardisierten Fragebogens nachuntersucht und die gewonnenen Daten bezüglich der Behandlungsverfahren und Therapieergebnisse ausgewertet. Eingeschlossen wurden Patienten bis zu einem Alter von einschließlich zwölf Jahren mit offenen kniegelenksnahen Wachstumsfugen zum Zeitpunkt der Operation, deren intraligamentäre VKB-Ruptur mit einer Kreuzbandersatzplastik versorgt worden war und deren Nachuntersuchung frühestens sechs Monate postoperativ erfolgte.

Im Rahmen einer systematischen Literaturanalyse wurden die Literaturdatenbanken Medline und Embase, die Cochrane Library, die Springer-Verlagsdatenbank und die ThiemeVerlagsdatenbank durchsucht. Die letzte Aktualisierung erfolgte am 17. Juli 2008. Die Suchbegriffe waren: "Anterior cruciate ligament, ACL, child, immature, pediatric, young, open growth plates, growth age" und "open physes". Einschlusskriterien waren die Versorgung einer intraligamentären VKB-Ruptur mit einer Kreuzbandersatzplastik oder Kreuzbandnaht und eine spätere Nachuntersuchung der Patienten. Außerdem war ein Alter der Patienten von maximal zwölf Jahren bei offenen kniegelenksnahen Wachstumsfugen zum Zeitpunkt der Operation und für die Vergleichsgruppe offene kniegelenksnahe Wachstumsfugen ohne Begrenzung des Alters Voraussetzung.

In den zehn Kliniken, die in die Multicenterstudie eingeschlossen waren, wurden 25 Patienten mit einem Durchschnittsalter von 10,4 (7 bis 12) Jahren behandelt. 23-mal wurde ein Hamstringtransplantat femoral und tibial fugenkreuzend, zweimal ein Patellasehnentransplantat fugenschonend eingebracht. Die Patienten hatten bei der Nachuntersuchung einen durchschnittlichen Lysholm-Score von 93,0 (42 bis 100) Punkten, 
beim $I K D C$ erreichten sieben die Kategorie A und dreizehn die Kategorie B; je einer erreichte die Kategorie C und D. Für zwei Patienten wurde kein Lysholm-Score, für drei kein IKDC erhoben. Die Messung der Bandlaxizität mit dem KT-1000 ergab eine Seitendifferenz von durchschnittlich $+1,0(-2$ bis +5$)$ mm auf der operierten Seite. Bei einem der Patienten kam es innerhalb des Nachuntersuchungszeitraums zu einer Reruptur des Bandes. Sechs Patienten entwickelten eine Beinlängendifferenz oder Achsenabweichung.

In der systematischen Literaturanalyse wurden insgesamt 59 Artikel mit 860 operativen Versorgungen von Patienten im Wachstumsalter erfasst. Von den Patienten bis zu einem Alter von zwölf Jahren wurden 37 mit einem Durchschnittsalter von 10,7 (2 bis 12) mit einer Kreuzbandersatzplastik versorgt. Ihr durchschnittlicher Lysholm-Score betrug 95,6 (84 bis 100) Punkte, keiner hatte eine Reruptur erlitten, zwei hatten eine Beinlängendifferenz oder Achsenabweichung entwickelt. Sechs Patienten wurden im Alter von durchschnittlich 9,8 (4 bis 12) Jahren mit einer Kreuzbandnaht versorgt. Für diese Gruppe wurde kein Lysholm-Score angegeben. Bei zweien trat eine Reruptur auf, eine Beinlängendifferenz oder Achsenabweichung wurde nicht beobachtet.

Von allen im Wachstumsalter erfassten Patienten wurden 769 mit einem Altersdurchschnitt von 13,8 (2 bis 17) Jahren mit einer Kreuzbandersatzplastik behandelt. Bei einem durchschnittlichen Lysholm-Score von 94,4 (62 bis 100) Punkten erlitten 30 (3,9 Prozent) von ihnen eine Reruptur und 29 (3,8 Prozent) eine Beinlängendifferenz oder Achsenabweichung. Bei 91 Patienten mit einem Alter von durchschnittlich 12,8 (4 bis 16) Jahren wurde das rupturierte Kreuzband genäht. Der durchschnittliche Lysholm-Score bei der Nachuntersuchung betrug bei diesen Patienten 79,6 Punkte. Siebenmal kam es zu einer Reruptur; eine Beinlängendifferenz oder Achsenabweichung trat nicht auf.

Bei der Betrachtung und Interpretation der Ergebnisse aus der Multicenterstudie und der systematischen Literaturanalyse nach einzelnen Untergruppen zeigte die Kreuzbandnaht bei Patienten im Alter von bis zu zwölf Jahren ähnlich schlechte Ergebnisse wie bei älteren Patienten und war der Kreuzbandersatzplastik unterlegen.

Fugenkreuzende und fugenschonende Operationstechniken führten sowohl bei den bis zu zwölf Jahren alten als auch bei allen Patienten im Wachstumsalter zu ähnlich guten klinischen Ergebnissen. Die Rate an Beinlängendifferenzen oder Achsenabweichungen war jedoch bei den fugenschonenden Techniken deutlich höher als bei den fugenkreuzenden. Fugenschonende Operationstechniken scheinen daher eher nachteilig zu sein. 
Zwischen den verwendeten Transplantaten (Patellasehne, Hamstring, Quadrizepssehne, Fascia lata und Allograft) und auch zwischen gelenknaher und gelenkferner Transplantatfixierung wurden in der Gruppe der bis zu zwölf Jahren alten Patienten und in der Gruppe aller Patienten im Wachstumsalter keine wesentlichen Unterschiede in den Ergebnissen festgestellt. Eine klare Empfehlung für ein bestimmtes Transplantat kann daher nicht gegeben werden. Die gelenknahe Transplantatbefestigung scheint trotz räumlicher Nähe des Implantationsmaterials zur Wachstumsfuge nicht von Nachteil zu sein.

Hinsichtlich der Technik zur Bohrung des femoralen Knochenkanals war der anteromediale Zugangsweg nicht mit einer höheren Rate an Beinlängendifferenzen oder Achsenabweichungen assoziiert als der transtibiale Zugang. Trotz der vermutlich flächenmäßig größeren Verletzung der Wachstumsfuge durch den flacheren Penetrationswinkel scheint daher die Kanalanlage über den anteromedialen Zugang vertretbar.

Insgesamt lassen sich für Patienten im Alter von bis zu zwölf Jahren mit einer Kreuzbandplastik gute und sehr gute klinische Ergebnisse erzielen. Die Gefahr, mit der Operation eine irreversible Beinlängendifferenz oder Achsenabweichung zu verursachen, ist jedoch beachtlich und bei Patienten bis zu zwölf Jahren vermutlich größer als bei älteren Patienten, die sich kurz vor dem Ende ihres Wachstums befinden.

Dass besonders die Patienten der Multicenterstudie eine hohe Rate an Beinlängendifferenzen oder Achsenabweichungen aufwiesen, spricht möglicherweise dafür, dass sonst viele dieser postoperativen Wachstumsstörungen bei beschwerdefreien Patienten unentdeckt bleiben, wenn von den Untersuchern nicht gezielt nach ihnen gefahndet wird.

Die Gefahr eines Fehlwachstums muss durch Einhalten mehrerer technischer Empfehlungen so weit wie irgend möglich minimiert werden. Auch unter Berücksichtigung aller technischen Aspekte kann es in Einzelfällen zu Wachstumsstörungen kommen. Daher sollten nach jeder Kreuzbandplastik regelmäßig postoperative Kontrollen bis zum Abschluss des Wachstums erfolgen. 


\section{Anhang}

\subsection{Nachuntersuchungsbogen der Multicenterstudie}

\section{Nachuntersuchungsbogen Kreuzbandruptur im Wachstumsalter \\ AG Arthroskopische Chirurgie der DGU}

\section{Individuelle Daten}

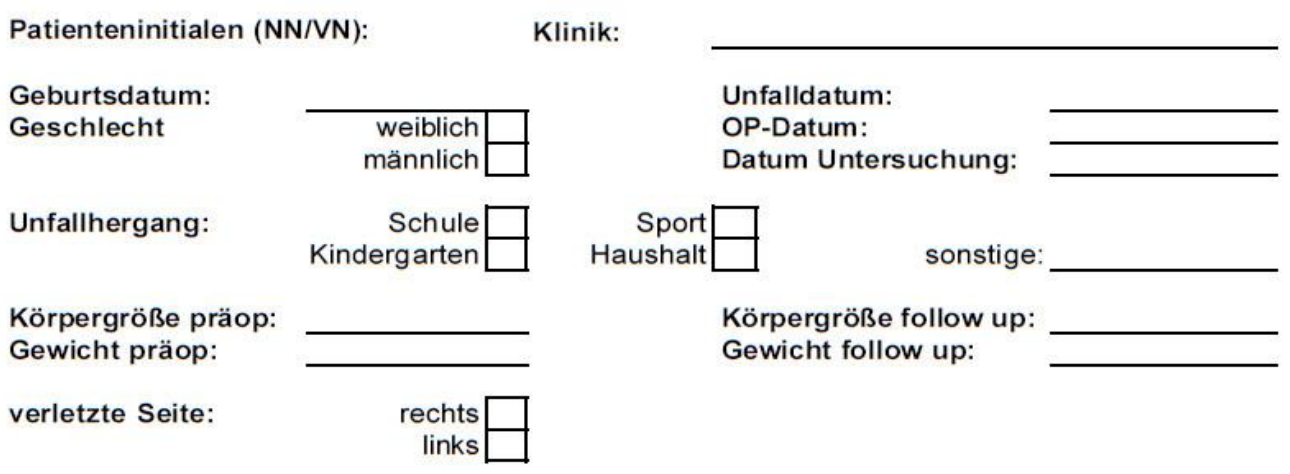

\section{Operative Daten}

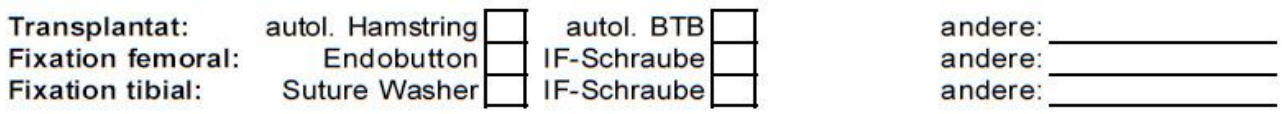

\begin{tabular}{|c|c|c|}
\hline $\begin{array}{ll}\text { Kanallage: } & \text { femoral transepiphysär } \\
& \text { tibial transepiphysär }\end{array}$ & $\begin{array}{l}\text { andere: } \\
\text { andere: }\end{array}$ & \\
\hline
\end{tabular}

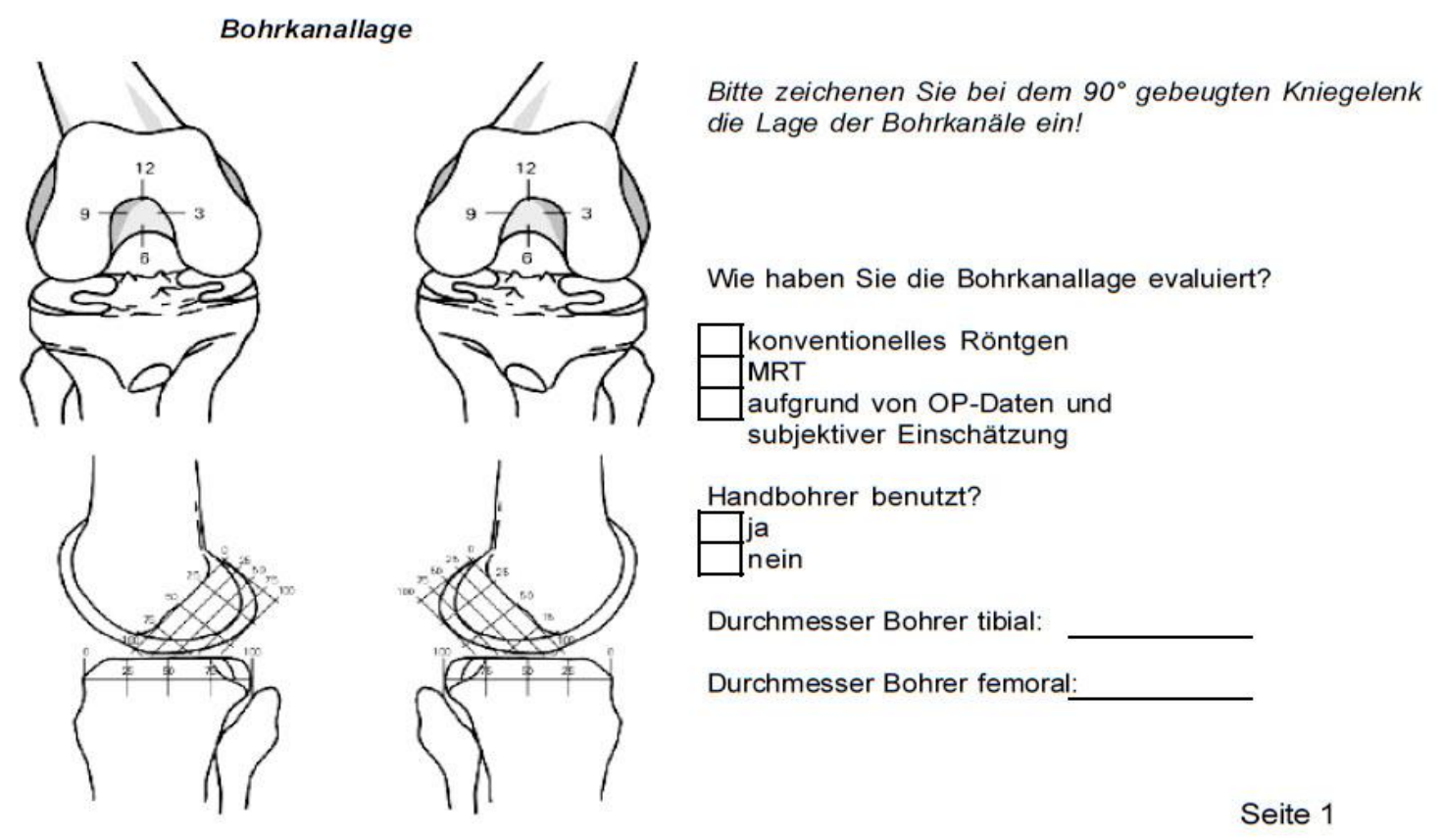

Abbildung 12: Nachuntersuchungsbogen der Multicenterstudie, Seite 1 


\section{Begleitverletzungen}

Begleitverletzungen:

Therapie Begleitverletzungen:

\section{Nachbehandlung}

Teilbelastung

Orthese

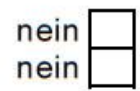

ja wenn ja, wie lange:

ja $\square$ wenn ja, wie lange:

KG

verordnete Sportpause

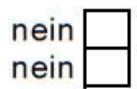

ja $\square$ wenn ja, wie lange:

ja $\square$ wenn ja, wie lange:

Dauer tatsächliche Sportpause:

\section{Nachuntersuchung}

Beinlängendifferenz präop

Beinlängendifferenz follow up
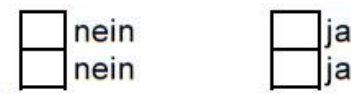

Ausmaß:

(jeweils klinisch, Brettchenmethode)

Ausmaß:

Varusabweichung (klinisch)

Valgusabweichung (klinisch)

genu recurvatum (klinisch)
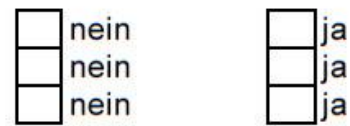

andere klinische Fehlstellung:

MRT

Fehlwachstum im MRT

degenerative Veränderungen

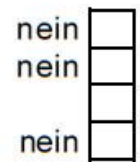

ja

ja $\square$

wenn ja, welche:

Ausmaß:

Ausmaß:

Ausmaß:

Röntgen Knie in $2 \mathrm{E}$

Auffälligkeiten?

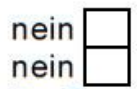

ja

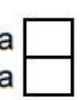

Datum:

wenn ja, bitte exakte Beschreibung:

\section{KT-1000 (falls vorhanden)}

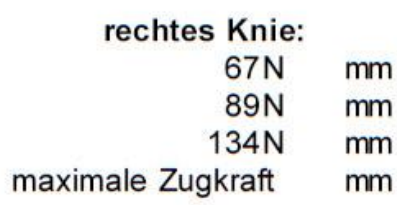

nein linkes Knie:

$67 \mathrm{~N} \mathrm{~mm}$

$89 \mathrm{~N} \mathrm{~mm}$

$134 \mathrm{~N} \mathrm{~mm}$

maximale Zugkraft $\mathrm{mm}$

Reruptur?

Komplikationen (z.B. Meniskusläsion im Verlauf, Knorpelschäden, etc):

Abbildung 13: Nachuntersuchungsbogen der Multicenterstudie, Seite 2 


\section{Lysholm-Score}

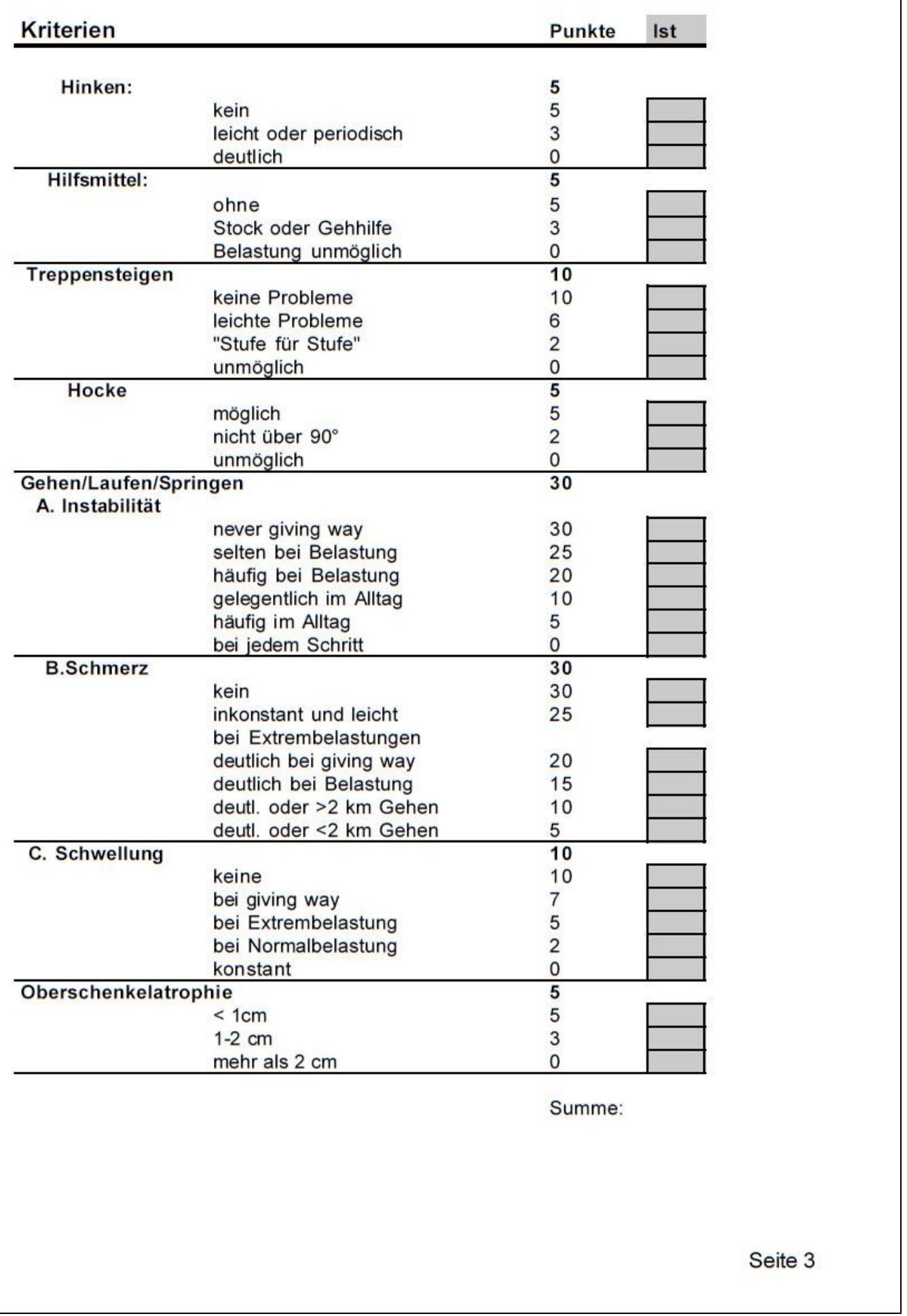

Abbildung 14: Nachuntersuchungsbogen der Multicenterstudie, Seite 3 


\section{Formblatt zur Untersuchung des Kniegelenks} nach IKDC 2000

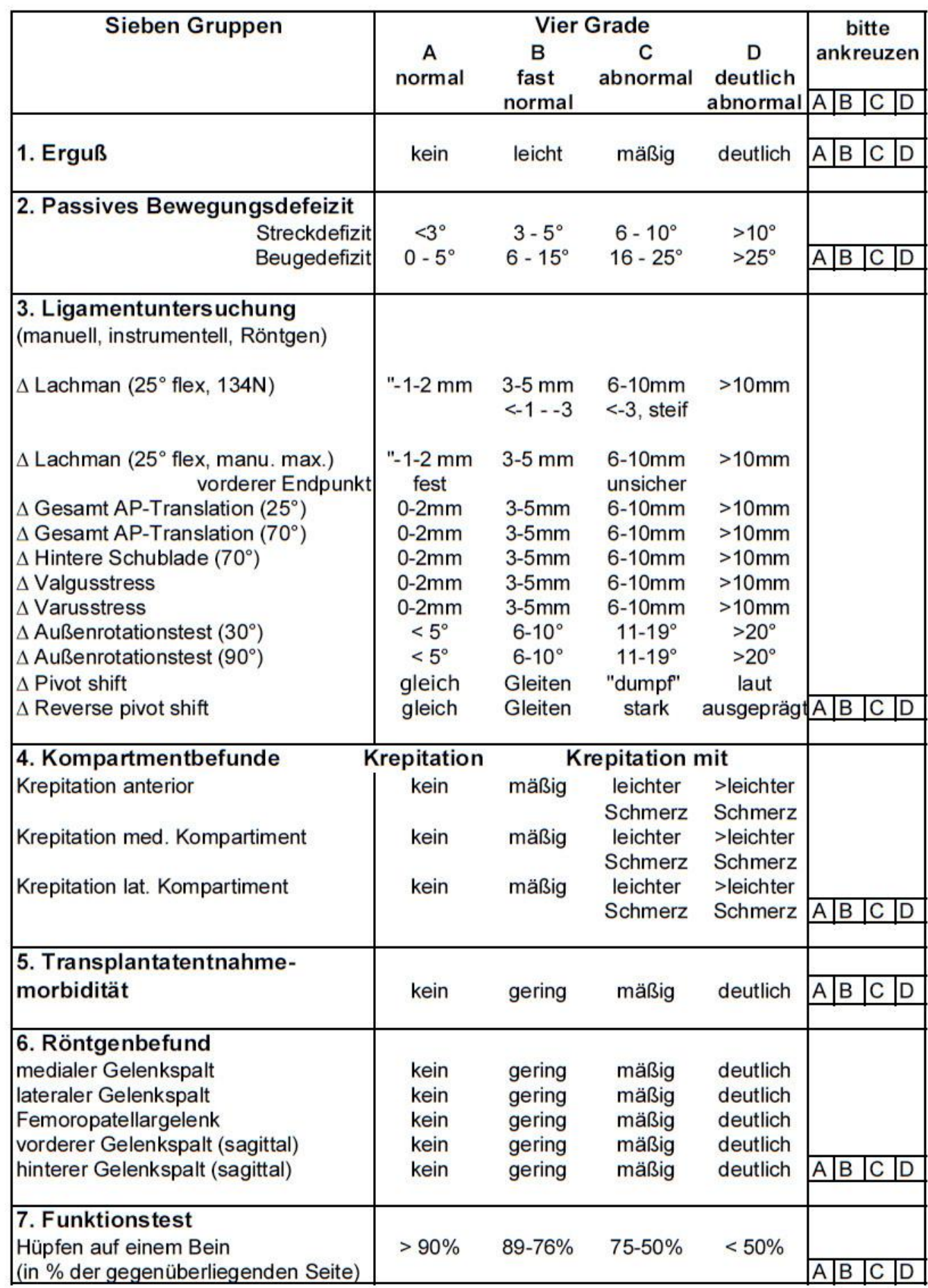

Abbildung 15: Nachuntersuchungsbogen der Multicenterstudie, Seite 4 


\subsection{Daten der Multicenterstudie}

Tabelle 16: Daten der Multicenterstudie

\begin{tabular}{|c|c|c|c|c|c|c|c|c|c|}
\hline Nr. & Klinik & Geburtsdatum & Alter & Geschl. & $\begin{array}{c}\text { Intervall } \\
\text { (Tage) }\end{array}$ & $\begin{array}{c}\text { Zeitraum von OP bis } \\
\text { Nachuntersuchung (Tage) }\end{array}$ & Unfallhergang & $\begin{array}{c}\text { Größe } \\
\text { präoperativ }\end{array}$ & $\begin{array}{c}\text { Größe } \\
\text { follow up }\end{array}$ \\
\hline 1 & Friederikenstift Hannover & 03.12 .1996 & 10 & $\mathrm{w}$ & 27 & 232 & Fahrradsturz & 138 & 140 \\
\hline 2 & Sportklinik Stuttgart & 17.08 .1997 & 9 & $\mathrm{~m}$ & 20 & 383 & Schulsport & k.A. & 166 \\
\hline 3 & Universitätsklinik Göttingen & 05.11 .1998 & 9 & $\mathrm{~m}$ & 69 & 243 & Sport & 130 & 140 \\
\hline 4 & Kath.Klinikum Koblenz & 16.01 .1999 & 8 & $\mathrm{~m}$ & k.A. & 522 & k.A. & 143 & 146 \\
\hline 5 & Kath.Klinikum Koblenz & 25.03 .1995 & 12 & $\mathrm{w}$ & k.A. & 461 & k.A. & 145 & 153 \\
\hline 6 & Kath.Klinikum Koblenz & 16.05 .1995 & 11 & $\mathrm{~m}$ & k.A. & 693 & k.A. & 158 & 168 \\
\hline 7 & MLK-Berlin & 17.07 .1986 & 12 & $\mathrm{~m}$ & 63 & 1232 & Sport & 165 & 182 \\
\hline 8 & MLK-Berlin & 06.05 .1989 & 12 & $\mathrm{~W}$ & 305 & 286 & Sport & 168 & 176 \\
\hline 9 & Sportklinik Stuttgart & 11.08 .1997 & 10 & $\mathrm{~m}$ & 52 & 278 & Sport & k.A. & 147 \\
\hline 10 & Universitätsklinik Freiburg & 29.01 .1995 & 12 & $\mathrm{w}$ & 95 & 435 & Sport & 156 & 160 \\
\hline 11 & Universitätsklinik Freiburg & 21.06 .2000 & 7 & $\mathrm{~m}$ & 92 & 543 & Sport & 120 & 136 \\
\hline 12 & MH-Hannover & 11.08 .1995 & 11 & $\mathrm{~m}$ & 25 & 916 & Sport & k.A. & 172 \\
\hline 13 & Uniklinik Gießen & 26.03 .1998 & 9 & $\mathrm{w}$ & 171 & 403 & Schulsport & 139 & 145 \\
\hline 14 & ARTICO Sportklinik V.-S. & k.A. & 11 & $\mathrm{~m}$ & 180 & 990 & Sport & k.A. & 168 \\
\hline 15 & ARTICO Sportklinik V.-S. & k.A. & 11 & $\mathrm{~m}$ & 7 & 370 & Sport & k.A. & k.A. \\
\hline 16 & ARTICO Sportklinik V.-S. & k.A. & 11 & $\mathrm{w}$ & 120 & 805 & Sport & k.A. & k.A. \\
\hline 17 & Universitätsklinik Göttingen & k.A. & 9 & $\mathrm{~m}$ & 42 & 365 & Sport & k.A. & k.A. \\
\hline 18 & MH-Hannover & 05.05 .1992 & 12 & $\mathrm{w}$ & k.A. & 2247 & Sport & 130 & 176 \\
\hline 19 & Marienkrankenhaus Kaiserswerth & 25.03 .1998 & 11 & $\mathrm{w}$ & k.A. & 492 & k.A. & 155 & 164 \\
\hline 20 & Marienkrankenhaus Kaiserswerth & 07.03 .1998 & 11 & $\mathrm{~m}$ & 81 & 549 & Fahrradsturz & 158 & 170 \\
\hline 21 & Marienkrankenhaus Kaiserswerth & 12.04 .1999 & 10 & $\mathrm{w}$ & k.A. & 615 & Sport & 150 & 154 \\
\hline 22 & Marienkrankenhaus Kaiserswerth & 30.10 .2000 & 8 & $\mathrm{~m}$ & 31 & 742 & Sport & 145 & 148 \\
\hline 23 & Marienkrankenhaus Kaiserswerth & 30.05 .1999 & 11 & $\mathrm{~m}$ & k.A. & 231 & Sport & 153 & 153 \\
\hline 24 & Marienkrankenhaus Kaiserswerth & 29.09 .1997 & 11 & $\mathrm{~m}$ & 39 & 716 & Sport & 152 & 155 \\
\hline 25 & Marienkrankenhaus Kaiserswerth & 07.01 .1999 & 11 & $\mathrm{~m}$ & k.A. & 346 & Sport & 152 & 154 \\
\hline
\end{tabular}




\begin{tabular}{|c|c|c|c|c|c|c|c|c|}
\hline Nr. & $\begin{array}{c}\text { Größenwachstum } \\
\text { (cm) }\end{array}$ & $\begin{array}{c}\text { Gewicht } \\
\text { präoperativ }\end{array}$ & $\begin{array}{l}\text { Gewicht } \\
\text { follow up }\end{array}$ & $\begin{array}{l}\text { Gewichts- } \\
\text { zunahme }\end{array}$ & Seite & Transplantat & Fixation femoral & Fixation tibial \\
\hline 1 & 2 & 36 & 37 & 1 & li. & autol. Hamstring & Transfixation & IF-Schraube \\
\hline 2 & k.A. & k.A. & 55 & k.A. & re. & autol. Hamstring & Endobutton & Ankerschraube \\
\hline 3 & 10 & k.A. & 30 & k.A. & re. & autol. Hamstring & Endobutton & Transfixation \\
\hline 4 & 3 & 35 & 36 & 1 & li. & autol. Hamstring & Endobutton & Suture Washer \\
\hline 5 & 8 & 32 & 37 & 5 & li. & autol. Hamstring & Endobutton & Titankrampe \\
\hline 6 & 10 & 46 & 61 & 15 & li. & autol. Hamstring & Endobutton & Suture Washer \\
\hline 7 & 17 & k.A. & k.A. & k.A. & li. & autol. BTB & Knochenblock in Kanal verkeilt & Naht an Periost + Schraube \\
\hline 8 & 8 & k.A. & k.A. & k.A. & li. & autol. BTB & Knochenblock in Kanal verkeilt & Naht an Periost \\
\hline 9 & k.A. & k.A. & 32,5 & k.A. & li. & autol. Hamstring & Endobutton & Ankerschraube \\
\hline 10 & 4 & 48 & 49 & 1 & re. & autol. Hamstring & IF-Schraube & IF-Schraube \\
\hline 11 & 16 & 20 & 27,5 & 7,5 & li. & autol. Hamstring & IF-Schraube & IF-Schraube \\
\hline 12 & k.A. & k.A. & 70 & k.A. & li. & autol. Hamstring & Endobutton & IF-Schraube \\
\hline 13 & 6 & 47 & 51 & 4 & re. & autol. Hamstring & Endobutton & Suture Washer \\
\hline 14 & k.A. & k.A. & k.A. & k.A. & li. & autol. Hamstring & press fit (fremdmaterialfrei) & press fit (fremdmaterialfrei) \\
\hline 15 & k.A. & k.A. & k.A. & k.A. & re. & autol. Hamstring & press fit (fremdmaterialfrei) & press fit (fremdmaterialfrei) \\
\hline 16 & k.A. & k.A. & k.A. & k.A. & re. & autol. Hamstring & press fit (fremdmaterialfrei) & press fit (fremdmaterialfrei) \\
\hline 17 & 8 & k.A. & k.A. & 4 & re. & autol. Hamstring & Endobutton & Suture Washer \\
\hline 18 & 46 & 40 & 70 & 30 & li. & autol. Hamstring & Endobutton & IF-Schraube \\
\hline 19 & 9 & 45 & 53 & 8 & re. & autol. Hamstring & Endobutton & Krampen \\
\hline 20 & 12 & 44 & 50 & 6 & re. & autol. Hamstring & Endobutton & Suture Washer \\
\hline 21 & 4 & 32 & 37 & 5 & li. & autol. Hamstring & Endobutton & Pollerschraube \\
\hline 22 & 3 & 38,5 & 44 & 5,5 & li. & autol. Hamstring & Endobutton & Schraube + Krampe \\
\hline 23 & 0 & 42 & 48 & 6 & li. & autol. Hamstring & Endobutton & Pollerschraube \\
\hline 24 & 3 & 44 & 48 & 4 & li. & autol. Hamstring & Endobutton & Ankerschraube \\
\hline 25 & 2 & 36 & 40 & 4 & li. & autol. Hamstring & Endobutton & Suture Washer \\
\hline
\end{tabular}


6. Anhang

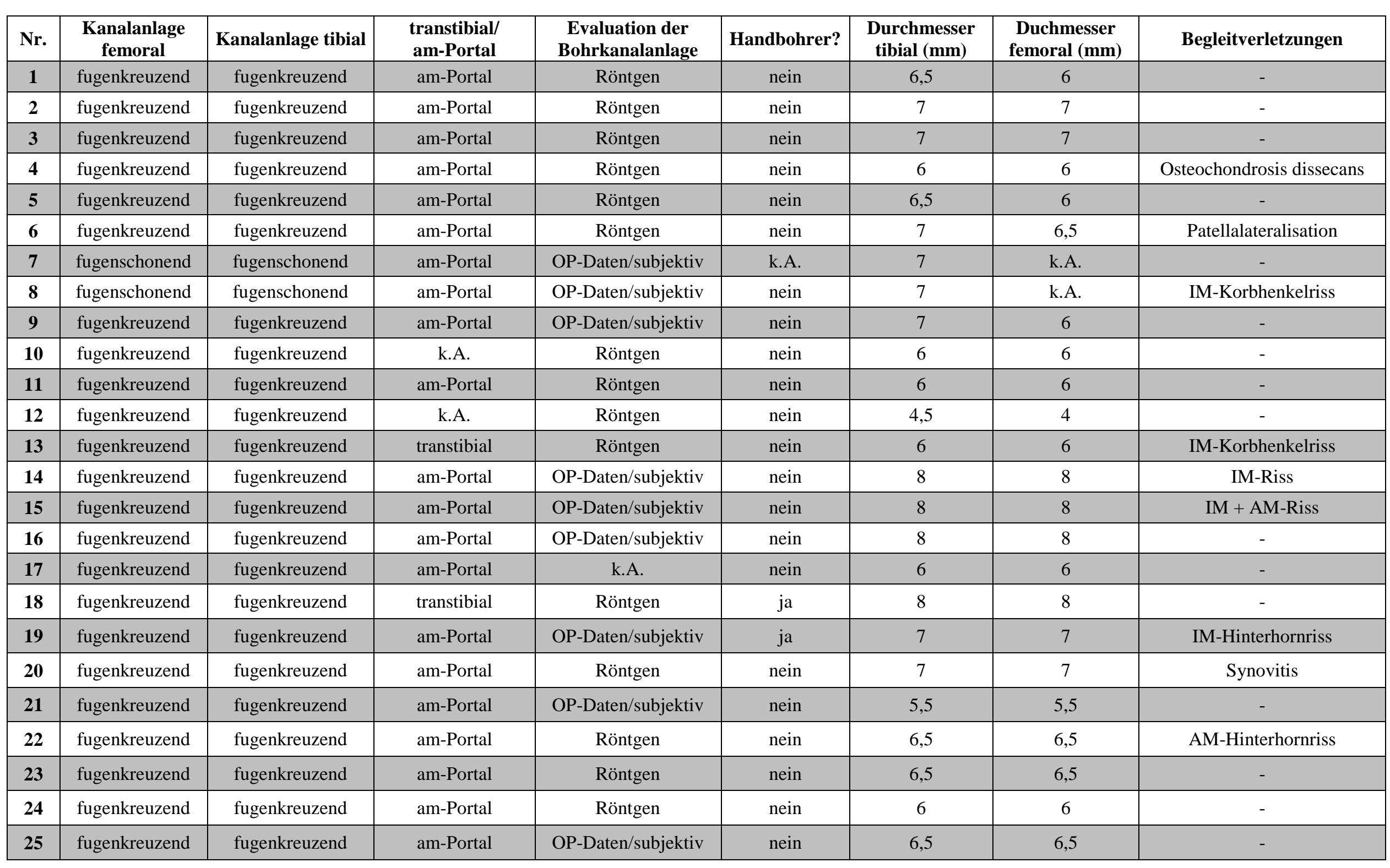




\begin{tabular}{|c|c|c|c|c|c|c|c|c|}
\hline Nr. & $\begin{array}{c}\text { Therapie der } \\
\text { Begleitverletzungen }\end{array}$ & KG & $\begin{array}{l}\text { Teilbelastung } \\
\text { (Wochen) }\end{array}$ & $\begin{array}{c}\text { Orthese } \\
\text { (Wochen) }\end{array}$ & $\begin{array}{c}\text { verordnete } \\
\text { Sportpause (Mo) } \\
\end{array}$ & $\begin{array}{c}\text { tatsächliche } \\
\text { Sportpause (Mo) }\end{array}$ & $\begin{array}{c}\text { Beinlängendiff. } \\
\text { präoperativ }\end{array}$ & $\begin{array}{c}\text { Beinlängendiff. } \\
\text { postoperativ }\end{array}$ \\
\hline 2 & - & nein & 2 & 6 & 6 & 6 & nein & nein \\
\hline 4 & keine & $6 \mathrm{Wo}$ & 3 & 6 & 1,5 & 2 & nein & nein \\
\hline 5 & - & $3 \mathrm{Mo}$ & 6 & 12 & 3 & k.A. & nein & nein \\
\hline 6 & arthr. Lat. Patellarelease & $6 \mathrm{Mo}$ & 4 & 12 & 6 & 6 & nein & nein \\
\hline 8 & Naht & k.A. & 6 & 6 & 3 & k.A. & nein & $-1 \mathrm{~cm}$ \\
\hline 9 & - & $3 \mathrm{Mo}$ & 2 & 6 & 6 & 6 & nein & nein \\
\hline 10 & - & $12 \mathrm{Wo}$ & 2 & 6 & 12 & k.A. & nein & nein \\
\hline 11 & - & ja & Ja, k.A über Dauer & 6 & 12 & k.A. & nein & nein \\
\hline 12 & - & $12 \mathrm{Mo}$ & 6 & 6 & 6 & 2 & $-1,5 \mathrm{~cm}$ & $-2,5 \mathrm{~cm}$ \\
\hline 13 & arthroskop. Refixation & $6 \mathrm{Mo}$ & 4 & 12 & 6 & k.A. & nein & nein \\
\hline 17 & - & nein & 4 & 6 & 6 & 6 & nein & nein \\
\hline 18 & - & $10 \mathrm{Wo}$ & 3 & 6 & 3 & 6 & nein & nein \\
\hline 19 & Naht & ja & 6 & 12 & 8 & 16 & nein & nein \\
\hline 20 & partielle Synovektomie & ja & direkt Vollbelastung & 6 & 8 & k.A. & nein & nein \\
\hline 21 & - & $8 \mathrm{Wo}$ & 6 & 12 & 8 & 4,5 & nein & nein \\
\hline 22 & AM-HH-Teilresektion & $8 \mathrm{Wo}$ & 6 & 12 & 8 & 8 & nein & nein \\
\hline 23 & - & $7 \mathrm{Mo}$ & 6 & 12 & 8 & 5 & nein & nein \\
\hline 24 & - & ja & direkt Vollbelastung & 6 & 8 & 8 & nein & nein \\
\hline 25 & - & $8 \mathrm{Wo}$ & direkt Vollbelastung & 10 & 6 & 6 & nein & nein \\
\hline
\end{tabular}


6. Anhang

\begin{tabular}{|c|c|c|c|c|c|c|c|c|c|}
\hline Nr. & Varusabweichung & Valgusabweichung & $\begin{array}{c}\text { Genu } \\
\text { recurvatum }\end{array}$ & $\begin{array}{c}\text { andere klinische } \\
\text { Fehlstellung }\end{array}$ & MRT & $\begin{array}{c}\text { Fehlwachstum } \\
\text { im MRT }\end{array}$ & $\begin{array}{c}\text { degenerative } \\
\text { Veränderungen }\end{array}$ & $\begin{array}{c}\text { Röntgen in } \\
\text { zwei Ebenen }\end{array}$ & Auffälligkeiten \\
\hline 1 & nein & nein & nein & nein & & & nein & $\mathrm{ja}$ & nein \\
\hline 2 & nein & nein & nein & nein & & & nein & & \\
\hline 3 & nein & nein & nein & nein & & & nein & & \\
\hline 4 & nein & nein & nein & nein & & & nein & & \\
\hline 5 & nein & nein & $10^{\circ}$ & nein & & & nein & & \\
\hline 6 & nein & nein & nein & nein & & & nein & & \\
\hline 7 & nein & nein & nein & nein & & & nein & & \\
\hline 8 & nein & nein & nein & nein & & & nein & $\mathrm{ja}$ & nein \\
\hline 9 & nein & nein & nein & nein & & & nein & & \\
\hline 10 & nein & nein & nein & nein & ja & nein & nein & & \\
\hline 11 & nein & nein & nein & nein & & & nein & & \\
\hline 12 & nein & nein & $10^{\circ}$ & nein & & & nein & & \\
\hline 13 & nein & nein & nein & nein & ja & nein & nein & & \\
\hline 14 & nein & $8^{\circ}$ & $10^{\circ}$ & nein & & & Crepitatio retropat. & ja & nein \\
\hline 15 & nein & nein & nein & nein & & & nein & ja & nein \\
\hline 16 & nein & nein & $5^{\circ}$ & nein & & & Crepitatio retropat. & & \\
\hline 17 & nein & nein & nein & nein & & & nein & & \\
\hline 18 & nein & nein & nein & nein & ja & nein & nein & ja & nein \\
\hline 19 & nein & nein & nein & nein & & & nein & & \\
\hline 20 & nein & nein & nein & nein & & & nein & & \\
\hline 21 & nein & nein & nein & nein & & & nein & & \\
\hline 22 & nein & nein & nein & nein & & & nein & & \\
\hline 23 & nein & nein & nein & nein & & & nein & & \\
\hline 24 & nein & nein & nein & nein & & & nein & & \\
\hline 25 & nein & nein & nein & nein & & & nein & & \\
\hline
\end{tabular}




\begin{tabular}{|c|c|c|c|c|c|c|c|}
\hline Nr. & $\begin{array}{l}\text { KT-1000 re. } \\
\text { (mm) }\end{array}$ & KT-1000 li. (mm) & Seitendifferenz $(\mathbf{m m})$ & Reruptur & $\begin{array}{c}\text { weitere } \\
\text { Komplikationen }\end{array}$ & Lysholm-Score & $I K D C$ \\
\hline 1 & k.A. & k.A. & k.A. & nein & nein & 100 & A \\
\hline 2 & k.A. & k.A. & k.A. & nein & nein & 95 & B \\
\hline 3 & k.A. & k.A. & k.A. & nein & nein & 95 & B \\
\hline 4 & k.A. & k.A. & k.A. & nein & nein & 95 & B \\
\hline 5 & 4,5 & 5,5 & 1 & nein & nein & 95 & B \\
\hline 6 & 5,5 & 5 & $-0,5$ & nein & nein & 95 & B \\
\hline 7 & 10 & 12 & 2 & nein & nein & k.A. & $\mathrm{C}$ \\
\hline 8 & 7 & 10 & 3 & nein & Revision bei IM-Schaden & k.A. & $\mathrm{B}$ \\
\hline 9 & k.A. & k.A. & k.A. & nein & nein & 98 & A \\
\hline 10 & 10 & 11 & -1 & nein & nein & 100 & $\mathrm{~B}$ \\
\hline 11 & 12 & 11 & -1 & nein & nein & 100 & B \\
\hline 12 & 10 & 15 & 5 & nein & nein & 95 & B \\
\hline 13 & k.A. & k.A. & k.A. & nein & nein & 100 & A \\
\hline 14 & 10 & 11 & 1 & nein & nein & 70 & k.A. \\
\hline 15 & 3 & 4 & -1 & nein & nein & 87 & k.A. \\
\hline 16 & 8 & 7 & 1 & nein & nein & 84 & k.A. \\
\hline 17 & k.A. & k.A. & 1,5 & nein & nein & 100 & $\mathrm{~A}$ \\
\hline 18 & 8 & 12 & 4 & nein & nein & 90 & B \\
\hline 19 & 8 & 10 & -2 & nein & nein & 97 & B \\
\hline 20 & 8 & 6 & 2 & nein & nein & 100 & B \\
\hline 21 & 8 & 8 & 0 & nein & nein & 100 & A \\
\hline 22 & 9 & 8 & -1 & nein & nein & 100 & $\mathrm{~A}$ \\
\hline 23 & 4 & 6 & 2 & nein & nein & 100 & B \\
\hline 24 & 6 & 8 & 2 & $\mathrm{ja}$ & nein & 42 & D \\
\hline 25 & 6 & 6 & 0 & nein & nein & 100 & A \\
\hline
\end{tabular}




\subsection{Daten der Patienten bis zu zwölf Jahren aus der systematischen Literaturanalyse}

Tabelle 17: Daten der Patienten bis zu zwölf Jahren aus der systematischen Literaturanalyse

\begin{tabular}{|c|c|c|c|c|c|c|c|c|c|}
\hline Autor & $\begin{array}{l}\text { Pati- } \\
\text { enten }\end{array}$ & Art der Versorgung der VKB-Ruptur & Alter & \begin{tabular}{|l|} 
Fixation \\
fem./tib.
\end{tabular} & Zugangsweg & Transplantat & \begin{tabular}{|l|} 
Lys- \\
holm \\
\end{tabular} & \begin{tabular}{c|} 
Re- \\
ruptur
\end{tabular} & Fehlwachstum \\
\hline \multirow[t]{2}{*}{ Aronowitz et al. 2000} & 2 & femoral und tibial fugenkreuzend & 11 & fern/fern & k.A. & Allograft & 96 & 0 & 0 \\
\hline & & femoral und tibial fugenkreuzend & 12 & fern/fern & k.A. & Allograft & 100 & 0 & 0 \\
\hline Bradley et al. 1979 & 1 & Kreuzbandnaht & 11 & - & - & - & k.A. & 0 & 0 \\
\hline \multirow[t]{3}{*}{ DeLee und Curtis 1983} & 3 & Kreuzbandnaht & 9 & - & - & - & k.A. & 0 & 0 \\
\hline & & Kreuzbandnaht & 11 & - & - & - & k.A. & 0 & 0 \\
\hline & & Kreuzbandnaht & 12 & - & - & - & k.A. & 0 & 0 \\
\hline Edwards und Grana 2001 & 1 & femoral und tibial fugenkreuzend & 11 & nah/nah & k.A. & Hamstring & 90 & 0 & 0 \\
\hline Gaudernak 1996 & 1 & Kreuzbandnaht & 12 & - & - & - & k.A. & 1 & 0 \\
\hline \multirow[t]{5}{*}{ Guzzanti et al. $2003 \mathrm{~b}$} & 5 & femoral und tibial fugenschonend & 11 & nah/fern & k.A. & Hamstring & k.A. & 0 & 0 \\
\hline & & femoral und tibial fugenschonend & 10 & nah/fern & k.A. & Hamstring & k.A. & 0 & 0 \\
\hline & & femoral und tibial fugenschonend & 12 & nah/fern & k.A. & Hamstring & k.A. & 0 & 0 \\
\hline & & femoral und tibial fugenschonend & 10 & nah/fern & k.A. & Hamstring & k.A. & 0 & 0 \\
\hline & & femoral und tibial fugenschonend & 11 & nah/fern & k.A. & Hamstring & k.A. & 0 & 0 \\
\hline Knorr et al. 2006 & 1 & femoral und tibial fugenschonend & 5 & nah/nah & k.A. & Patellasehne & 99 & 0 & 0 \\
\hline \multirow[t]{5}{*}{ Lipscomp und Anderson 1986} & 5 & tibial fugenkreuzend, femoral fugenschonend & 12 & fern/fern & k.A. & Hamstring & k.A. & 0 & Aussage unmögl. \\
\hline & & tibial fugenkreuzend, femoral fugenschonend & 11 & fern/fern & k.A. & Hamstring & k.A. & 0 & Aussage unmögl. \\
\hline & & tibial fugenkreuzend, femoral fugenschonend & 10 & fern/fern & k.A. & Hamstring & k.A. & 0 & Aussage unmögl. \\
\hline & & tibial fugenkreuzend, femoral fugenschonend & 12 & fern/fern & k.A. & Hamstring & k.A. & 0 & Aussage unmögl. \\
\hline & & tibial fugenkreuzend, femoral fugenschonend & 12 & fern/fern & k.A. & Hamstring & k.A. & 0 & Aussage unmögl. \\
\hline Lo et al. 1997 & 1 & tibial fugenkreuzend, femoral fugenschonend & 8 & fern/fern & k.A. & Hamstring & k.A. & 0 & 0 \\
\hline
\end{tabular}




\begin{tabular}{|c|c|c|c|c|c|c|c|c|c|}
\hline Autor & $\begin{array}{l}\text { Pati- } \\
\text { enten }\end{array}$ & Art der Versorgung der VKB-Ruptur & Alter & \begin{tabular}{|l|} 
Fixation \\
fem./tib.
\end{tabular} & Zugangsweg & Transplantat & \begin{tabular}{|l|} 
Lys- \\
holm \\
\end{tabular} & \begin{tabular}{|c|} 
Re- \\
ruptur
\end{tabular} & Fehlwachstum \\
\hline \multirow[t]{5}{*}{ Lukas et al. 2007} & 5 & femoral und tibial fugenkreuzend & 12 & fern/fern & anteromedial & Patellasehne & 92 & 0 & 0 \\
\hline & & femoral und tibial fugenkreuzend & 11 & fern/fern & anteromedial & Patellasehne & 100 & 0 & 0 \\
\hline & & femoral und tibial fugenkreuzend & 11 & fern/fern & anteromedial & Patellasehne & 100 & 0 & 0 \\
\hline & & femoral und tibial fugenkreuzend & 9 & fern/fern & anteromedial & Patellasehne & 90 & 0 & 0 \\
\hline & & femoral und tibial fugenkreuzend & 11 & fern/fern & anteromedial & Patellasehne & 98 & 0 & 0 \\
\hline Matava und Siegel 1997 & 1 & femoral und tibial fugenkreuzend & 12 & fern/fern & k.A. & Hamstring & 94 & 0 & 0 \\
\hline \multirow[t]{5}{*}{ Micheli et al. 1999} & 5 & femoral und tibial fugenschonend & 12 & fern/fern & k.A. & Fascia lata & 100 & 0 & 0 \\
\hline & & femoral und tibial fugenschonend & 12 & fern/fern & k.A. & Fascia lata & 93 & 0 & 0 \\
\hline & & femoral und tibial fugenschonend & 2 & fern/fern & k.A. & Fascia lata & 94 & 0 & $-20 \mathrm{~mm}$ \\
\hline & & femoral und tibial fugenschonend & 10 & fern/fern & k.A. & Fascia lata & 100 & 0 & 0 \\
\hline & & femoral und tibial fugenschonend & 11 & fern/fern & k.A. & Fascia lata & 95 & 0 & 0 \\
\hline Moksnes et al. 2008 & 1 & Plastik (k.A. ob fugenkreuzend o. nicht) & 12 & k.A. & k.A. & Hamstring & 84 & 0 & 0 \\
\hline Nakhostine et al. 1995 & 1 & femoral und tibial fugenschonend & 12 & fern/fern & k.A. & Fascia lata & k.A. & 0 & Genu recurvatum \\
\hline \multirow[t]{6}{*}{ Robert und Bonnard 1999} & 6 & femoral und tibial fugenschonend & 9 & nah/nah & anteromedial & Patellasehne & k.A. & 0 & 0 \\
\hline & & femoral und tibial fugenschonend & 11 & nah/nah & anteromedial & Patellasehne & k.A. & 0 & 0 \\
\hline & & femoral und tibial fugenschonend & 11 & nah/nah & anteromedial & Patellasehne & k.A. & 0 & 0 \\
\hline & & femoral und tibial fugenschonend & 12 & nah/nah & anteromedial & Patellasehne & k.A. & 0 & 0 \\
\hline & & femoral und tibial fugenschonend & 11 & nah/nah & anteromedial & Patellasehne & k.A. & 0 & 0 \\
\hline & & femoral und tibial fugenschonend & 11 & nah/nah & anteromedial & Patellasehne & k.A. & 0 & 0 \\
\hline \multirow[t]{3}{*}{ Sankar et al. 2006} & 3 & femoral und tibial fugenkreuzend & 11 & fern/fern & transtibial & Allograft (achilles) & 96 & 0 & 0 \\
\hline & & femoral und tibial fugenkreuzend & 12 & fern/fern & transtibial & Allograft (achilles) & 96 & 0 & 0 \\
\hline & & femoral und tibial fugenkreuzend & 12 & fern/fern & transtibial & Allograft (achilles) & 100 & 0 & 0 \\
\hline Schaefer et al. 1993 & 1 & Kreuzbandnaht & 4 & - & - & - & k.A. & 1 & 0 \\
\hline
\end{tabular}




\subsection{Beinlängendifferenzen und Achsenabweichungen aus der systematischen Literaturanalyse}

Tabelle 18: Beinlängendifferenzen und Achsenabweichungen aus der systematischen Literaturanalyse

\begin{tabular}{|c|c|c|c|c|c|c|c|c|}
\hline Nr. & Autor & $\begin{array}{c}\text { Alter } \\
\text { (bei OP) }\end{array}$ & Operationstechnik & Transplantat & \begin{tabular}{|c|} 
Fixierung: \\
femoral/tibial
\end{tabular} & $\begin{array}{c}\text { Anlage des } \\
\text { fem. Kanals }\end{array}$ & $\begin{array}{c}\text { Art des } \\
\text { Fehlwachstums }\end{array}$ & Grund des Fehlwachstums \\
\hline 1 & Anderson 2003 & k.A. & $\begin{array}{l}\text { femoral und tibial } \\
\text { fugenschonend }\end{array}$ & Hamstring & fern/fern & AM-Portal & $+10 \mathrm{~mm}$ (nur femoral) & k.A. \\
\hline 2 & Andrews et al. 1994 & k.A. & $\begin{array}{l}\text { femoral fugenschonend, } \\
\text { tibial fugenkreuzend }\end{array}$ & Allograft & fern/fern & k.A. & $-12 \mathrm{~mm}$ (nur femoral) & k.A. \\
\hline 3 & & k.A. & $\begin{array}{l}\text { femoral fugenschonend, } \\
\text { tibial fugenkreuzend }\end{array}$ & Hamstring & fern/fern & k.A. & +10 mm (nur femoral) & k.A. \\
\hline 4 & $\begin{array}{c}\text { Edwards und Grana } \\
2001\end{array}$ & 14 & $\begin{array}{l}\text { femoral und tibial } \\
\text { fugenkreuzend }\end{array}$ & Hamstring & nah/nah & k.A. & $-10 \mathrm{~mm}$ & k.A. \\
\hline 5 & \begin{tabular}{|} 
Koman und Sanders \\
1999
\end{tabular} & 14 & $\begin{array}{l}\text { femoral und tibial } \\
\text { fugenkreuzend }\end{array}$ & Hamstring & nah/fern & k.A. & $14^{\circ}$ valgus & $\begin{array}{c}\text { Fixierungsschraube kreuzte den } \\
\text { lateralen Teil der distalen } \\
\text { femoralen Wachstumsfuge }\end{array}$ \\
\hline 6 & $\begin{array}{l}\text { Lipscomp und } \\
\text { Anderson } 1986\end{array}$ & k.A. & $\begin{array}{l}\text { femoral fugenschonend, } \\
\text { tibial fugenkreuzend }\end{array}$ & Hamstring & fern/fern & k.A. & $+13 \mathrm{~mm}$ & k.A. \\
\hline 7 & & k.A. & $\begin{array}{l}\text { femoral fugenschonend, } \\
\text { tibial fugenkreuzend }\end{array}$ & Hamstring & fern/fern & k.A. & $-20 \mathrm{~mm}$ & $\begin{array}{c}\text { Klammern über } \\
\text { beiden Wachstumsfugen }\end{array}$ \\
\hline 8 & & k.A. & $\begin{array}{l}\text { femoral fugenschonend, } \\
\text { tibial fugenkreuzend }\end{array}$ & Hamstring & fern/fern & k.A. & $3^{\circ}$ valgus & k.A. \\
\hline 9 & $\begin{array}{l}\text { McIntosh et al. } \\
2006\end{array}$ & k.A. & $\begin{array}{l}\text { femoral und tibial } \\
\text { fugenkreuzend }\end{array}$ & Hamstring & fern/fern & transtibial & $+15 \mathrm{~mm}$ & k.A. \\
\hline 10 & Micheli et al. 1999 & 2 & $\begin{array}{l}\text { femoral und tibial } \\
\text { fugenschonend }\end{array}$ & Iliotibialband & fern/fern & $\begin{array}{c}\text { kein } \\
\text { Bohrkanal }\end{array}$ & $-20 \mathrm{~mm}$ & $\begin{array}{c}\text { Autor vermutet eine kongenitale } \\
\text { Anisomelie als Ursache für die } \\
\text { Beinlängendifferenz }\end{array}$ \\
\hline 11 & Nakostine et al. 1995 & 12 & $\begin{array}{l}\text { femoral und tibial } \\
\text { fugenschonend }\end{array}$ & Iliotibialband & fern/fern & $\begin{array}{c}\text { kein } \\
\text { Bohrkanal }\end{array}$ & recurvatum & k.A. \\
\hline 12 & & 14 & $\begin{array}{l}\text { femoral und tibial } \\
\text { fugenschonend }\end{array}$ & Iliotibialband & fern/fern & $\begin{array}{c}\text { kein } \\
\text { Bohrkanal } \\
\end{array}$ & recurvatum & k.A. \\
\hline 13 & & 14 & $\begin{array}{l}\text { femoral und tibial } \\
\text { fugenschonend }\end{array}$ & Iliotibialband & fern/fern & $\begin{array}{c}\text { kein } \\
\text { Bohrkanal }\end{array}$ & recurvatum & k.A. \\
\hline
\end{tabular}




\begin{tabular}{|c|c|c|c|c|c|c|c|c|}
\hline Nr. & Autor & $\begin{array}{c}\text { Alter } \\
\text { (bei OP) }\end{array}$ & Operationstechnik & Transplantat & $\begin{array}{c}\text { Fixierung: } \\
\text { femoral/tibial }\end{array}$ & $\begin{array}{c}\text { Anlage des } \\
\text { fem. Kanals }\end{array}$ & $\begin{array}{c}\text { Art des } \\
\text { Fehlwachstums } \\
\end{array}$ & $\begin{array}{c}\text { Grund des } \\
\text { Fehlwachstums }\end{array}$ \\
\hline 14 & & 15 & $\begin{array}{l}\text { femoral und tibial } \\
\text { fugenschonend }\end{array}$ & Iliotibialband & fern/fern & $\begin{array}{c}\text { kein } \\
\text { Bohrkanal }\end{array}$ & recurvatum & k.A. \\
\hline 15 & & 15 & $\begin{array}{l}\text { femoral und tibial } \\
\text { fugenschonend }\end{array}$ & Iliotibialband & fern/fern & $\begin{array}{c}\text { kein } \\
\text { Bohrkanal }\end{array}$ & recurvatum & k.A. \\
\hline 16 & Parker et al. 1994 & k.A. & $\begin{array}{l}\text { femoral und tibial } \\
\text { fugenschonend }\end{array}$ & Hamstring & fern/k.A. & $\begin{array}{c}\text { kein } \\
\text { Bohrkanal }\end{array}$ & $+10 \mathrm{~mm}$ & k.A. \\
\hline 17 & & k.A. & $\begin{array}{l}\text { femoral und tibial } \\
\text { fugenschonend }\end{array}$ & Hamstring & fern/k.A. & $\begin{array}{c}\text { kein } \\
\text { Bohrkanal }\end{array}$ & $+10 \mathrm{~mm}$ & k.A. \\
\hline 18 & & k.A. & $\begin{array}{l}\text { femoral und tibial } \\
\text { fugenschonend }\end{array}$ & Hamstring & fern/k.A. & $\begin{array}{c}\text { kein } \\
\text { Bohrkanal }\end{array}$ & $+10 \mathrm{~mm}$ & k.A. \\
\hline 19 & & k.A. & $\begin{array}{l}\text { femoral und tibial } \\
\text { fugenschonend }\end{array}$ & Hamstring & fern/k.A. & $\begin{array}{c}\text { kein } \\
\text { Bohrkanal }\end{array}$ & $+10 \mathrm{~mm}$ & k.A. \\
\hline 20 & & k.A. & $\begin{array}{l}\text { femoral und tibial } \\
\text { fugenschonend }\end{array}$ & Hamstring & fern/k.A. & $\begin{array}{c}\text { kein } \\
\text { Bohrkanal } \\
\end{array}$ & $+10 \mathrm{~mm}$ & k.A. \\
\hline 21 & Seil und Robert 2005 & k.A. & $\begin{array}{l}\text { femoral und tibial } \\
\text { fugenschonend }\end{array}$ & Patellasehne & k.A/nah & k.A. & $4^{\circ}$ valgus (femoral) & k.A. \\
\hline 22 & & k.A. & $\begin{array}{l}\text { femoral und tibial } \\
\text { fugenschonend }\end{array}$ & Patellasehne & k.A/nah & k.A. & $7^{\circ}$ valgus (femoral) & k.A. \\
\hline 23 & & k.A. & $\begin{array}{l}\text { femoral und tibial } \\
\text { fugenschonend }\end{array}$ & Patellasehne & k.A/nah & k.A. & $7^{\circ}$ varus (tibial) & k.A. \\
\hline 24 & & k.A. & $\begin{array}{l}\text { femoral und tibial } \\
\text { fugenschonend }\end{array}$ & Patellasehne & k.A/nah & k.A. & recurvatum (tibial) & k.A. \\
\hline 25 & & k.A. & $\begin{array}{l}\text { femoral und tibial } \\
\text { fugenschonend }\end{array}$ & Patellasehne & k.A/nah & k.A. & recurvatum (tibial) & k.A. \\
\hline 26 & Seon et al. 2005 & 15 & $\begin{array}{l}\text { femoral und tibial } \\
\text { fugenkreuzend }\end{array}$ & Hamstring & fern/nah & k.A. & $+10 \mathrm{~mm}$ & k.A. \\
\hline 27 & & 14 & $\begin{array}{l}\text { femoral und tibial } \\
\text { fugenkreuzend }\end{array}$ & Hamstring & fern/nah & k.A. & $+10 \mathrm{~mm}$ & k.A. \\
\hline 28 & & 15 & $\begin{array}{l}\text { femoral und tibial } \\
\text { fugenkreuzend }\end{array}$ & Hamstring & fern/nah & k.A. & $+10 \mathrm{~mm}$ & k.A. \\
\hline 29 & & 15 & $\begin{array}{l}\text { femoral und tibial } \\
\text { fugenkreuzend }\end{array}$ & Hamstring & fern/nah & k.A. & $-10 \mathrm{~mm}$ & k.A. \\
\hline
\end{tabular}




\section{Abkürzungsverzeichnis}

$\begin{array}{ll}\text { ACL } & \text { Anterior cruciate ligament } \\ \text { am } & \text { anteromedial } \\ \text { AM } & \text { Außenmeniskus } \\ \text { arthr. } & \text { arthroskopisch } \\ \text { autol. } & \text { autolog } \\ \text { BTB } & \text { Bone-Tendon-Bone } \\ \text { fem. } & \text { femoral } \\ \text { HH } & \text { Hinterhorn } \\ \text { IF-Schraube } & \text { Interferenzschraube } \\ \text { IKDC } & \text { international knee documentation committee } \\ \text { IM } & \text { Innenmeniskus } \\ \text { k.A. } & \text { keine Angabe } \\ \text { KG } & \text { Krankengymnastik } \\ \text { lat. } & \text { lateral } \\ \text { li. } & \text { links } \\ \text { Lig. } & \text { Ligamentum } \\ \text { m } & \text { männlich } \\ \text { Mo } & \text { Monat } \\ \text { MRT } & \text { Magnetresonanztomographie } \\ \mathrm{n} & \text { tibial } \\ \text { re. } & \text { Vorderes Kreuzband } \\ \text { tib. } & \text { NKB }\end{array}$




\section{Literaturverzeichnis}

Aichroth PM, Patel DV, Zorrilla P (2002): The natural history and treatment of rupture of the anterior cruciate ligament in children and adolescents. A prospective review. J Bone Joint Surg Br $\underline{84}, 38-41$.

Anderson AF (2003): Transepiphyseal replacement of the anterior cruciate ligament in skeletally immature patients. A preliminary report. J Bone Joint Surg Am $\underline{85-A}, 1255-1263$.

Andrews M, Noyes FR, Barber-Westin SD (1994): Anterior cruciate ligament allograft reconstruction in the skeletally immature athlete. Am J Sports Med 22, 48-54.

Andrish JT (2001): Anterior cruciate ligament injuries in the skeletally immature patient. Am J Orthop $\underline{30}, 103-110$.

Arbes S, Resinger C, Vecsei V, Nau T (2007): The functional outcome of total tears of the anterior cruciate ligament (ACL) in the skeletally immature patient. Int Orthop $\underline{31}$, 471-475.

Arndt S, Besch L, Havemann D (1999): [Internal knee injuries in children]. Unfallchirurg $\underline{102}, 619-624$.

Aronowitz ER, Ganley TJ, Goode JR, Gregg JR, Meyer JS (2000): Anterior cruciate ligament reconstruction in adolescents with open physes. Am J Sports Med 28, 168-175.

Attmanspacher W, Dittrich V, Stedtfeld HW (2002): [Revision of the anterior cruciate ligament in TransFix(R)- and OATS(R)-technique]. Zentralbl Chir 127, 855-860.

Attmanspacher W, Dittrich V, Stedtfeld HW (2003): [Results on treatment of anterior cruciate ligament rupture of immature and adolescents]. Unfallchirurg 106, 136-143.

Babb JR, Ahn JI, Azar FM, Canale ST, Beaty JH (2008): Transphyseal anterior cruciate ligament reconstruction using mesenchymal stem cells. Am J Sports Med 36, 1164-1170.

Bales CP, Guettler JH, Moorman CT (2004): Anterior cruciate ligament injuries in children with open physes: evolving strategies of treatment. Am J Sports Med 32, 1978-1985.

Behr CT, Potter HG, Paletta GA (2001): The relationship of the femoral origin of the anterior cruciate ligament and the distal femoral physeal plate in the skeletally immature knee. An anatomic study. Am J Sports Med 29, 781-787.

Benedetto KP, Glötzer W, Fink C (1996): Die vordere Kreuzbandplastik beim Jugendlichen mit noch offenen Epiphysenfugen. Acta Chir Austriaca 28, 23-25. 
Benjaminse A, Gokeler A, van der Schans CP (2006): Clinical diagnosis of an anterior cruciate ligament rupture: a meta-analysis. J Orthop Sports Phys Ther $\underline{36}$, 267-288.

Bisson LJ, Wickiewicz T, Levinson M, Warren R (1998): ACL reconstruction in children with open physes. Orthopedics 21, 659-663.

Bollen S, Pease F, Ehrenraich A, Church S, Skinner J Williams A (2008): Changes in the four-strand hamstring graft in anterior cruciate ligament reconstruction in the skeletallyimmature knee. J Bone Joint Surg Br 90, 455-459.

Bonnel F, Dimeglio A, Baldet P, Rabischong P (1984): Biomechanical activity of the growth plate. Clinical incidences. Anat Clin $\underline{6}, 53-61$.

Boyer P, Djian P, Christel P, Paoletti X, Degeorges R (2004): [Reliability of the KT-1000 arthrometer (Medmetric) for measuring anterior knee laxity: comparison with Telos in 147 knees]. Rev Chir Orthop Reparatrice Appar Mot 무, 757-764.

Bradley GW, Shives TC, Samuelson KM (1979): Ligament injuries in the knees of children. J Bone Joint Surg Am $\underline{61}$, 588-591.

Brief LP (1991): Anterior cruciate ligament reconstruction without drill holes. Arthroscopy $\underline{7}$, 350-357.

Butler DL, Noyes FR, Grood ES (1980): Ligamentous restraints to anterior-posterior drawer in the human knee. A biomechanical study. J Bone Joint Surg Am $\underline{62}$, 259-270.

Cabaud HE (1983): Biomechanics of the anterior cruciate ligament. Clin Orthop Relat Res $\underline{1983}, 26-31$.

Casper D, Herrmann D, Ekkernkamp A, Nowotny T (2006): Vordere Kreuzbandruptur bei offenen Wachstumsfugen - Therapie. Trauma Berufskrankh $\underline{8}, 38-41$.

Cohen M, Ferretti M, Quarteiro M, Marcondes FB, de Hollanda JP, Amaro JT, Abdalla RJ (2009): Transphyseal anterior cruciate ligament reconstruction in patients with open physes. Arthroscopy 25, 831-838.

Cook DJ, Mulrow CD, Haynes RB (1997): Systematic reviews: synthesis of best evidence for clinical decisions. Ann Intern Med 126, 376-380.

Daniel DM, Malcom LL, Losse G, Stone ML, Sachs R, Burks R (1985a): Instrumented measurement of anterior laxity of the knee. J Bone Joint Surg Am $\underline{67}, 720-726$. 
Daniel DM, Stone ML, Sachs R, Malcom L (1985b): Instrumented measurement of anterior knee laxity in patients with acute anterior cruciate ligament disruption. Am J Sports Med $\underline{13}$, 401-407.

Delay BS, Smolinski RJ, Wind WM, Bowman DS (2001): Current practices and opinions in ACL reconstruction and rehabilitation: results of a survey of the American Orthopaedic Society for Sports Medicine. Am J Knee Surg $\underline{14}$, 85-91.

DeLee JC, Curtis R (1983): Anterior cruciate ligament insufficiency in children. Clin Orthop Relat Res 1983, 112-118.

Dickersin K, Scherer R, Lefebvre C (1994): Identifying relevant studies for systematic reviews. BMJ $\underline{309}$, 1286-1291.

Dodds JA, Arnoczky SP (1994): Anatomy of the anterior cruciate ligament: a blueprint for repair and reconstruction. Arthroscopy $\underline{10}, 132-139$.

Dorizas JA, Stanitski CL (2003): Anterior cruciate ligament injury in the skeletally immature. Orthop Clin North Am 34, 355-363.

Edwards PH, Grana WA (2001): Anterior cruciate ligament reconstruction in the immature athlete: long-term results of intra-articular reconstruction. Am J Knee Surg 14, 232-237.

Edwards TB, Greene CC, Baratta RV, Zieske A, Willis RB (2001): The effect of placing a tensioned graft across open growth plates. A gross and histologic analysis. J Bone Joint Surg Am $\underline{\text { 83-A, }}$ 725-734.

Engebretsen L, Svenningsen S, Benum P (1988): Poor results of anterior cruciate ligament repair in adolescence. Acta Orthop Scand 59, 684-686.

Fankhauser F, Passler JM, Schippinger G, Boldin C, Scarpatetti M (2004): Tendon-to-bone healing of a quadrupled hamstring tendon graft fixed with biodegradable screws in an immature athlete: a radiologic, arthroscopic, histologic, and electromicroscopic investigation. Arthroscopy 20, 992-997.

Fauno P, Kaalund S (2005): Tunnel widening after hamstring anterior cruciate ligament reconstruction is influenced by the type of graft fixation used: a prospective randomized study. Arthroscopy 21, 1337-1341.

Fehnel DJ, Johnson R (2000): Anterior cruciate injuries in the skeletally immature athlete: a review of treatment outcomes. Sports Med 29, 51-63.

Ford LT, Key JA (1956): A study of experimental trauma to the distal femoral epiphysis in rabbits. J Bone Joint Surg Am $\underline{38-A}$, 84-92. 
Friederich NF, Müller W, O'Brien WR (1992): [Clinical application of biomechanic and functional anatomical findings of the knee joint]. Orthopade 21, 41-50.

Friedman RL, Jackson DW (1996): Magnetic resonance imaging of the anterior cruciate ligament: current concepts. Orthopedics $\underline{19}, 525-532$.

Fuchs R, Wheatley W, Uribe JW, Hechtman KS, Zvijac JE, Schurhoff MR (2002): Intraarticular anterior cruciate ligament reconstruction using patellar tendon allograft in the skeletally immature patient. Arthroscopy $\underline{18}, 824-828$.

Gaudernak T (1996): Operationsindikation für extraartikuläre Operationstechniken bei der vorderen Kreuzbandinsuffizienz bei Kindern und Jugendlichen. Acta Chir Austriaca 28 (Suppl.120), 21-23.

Gaulrapp H, Haus J (2003): Ergebnisse verschiedener operativer Behandlungsverfahren bei vorderen Kreuzbandverletzungen im Kindes- und Jugendalter: Stabilität ohne Wachstumsfehler. Arthroskopie 16, 239-251.

Gaulrapp HM, Haus J (2006): Intraarticular stabilization after anterior cruciate ligament tear in children and adolescents: results 6 years after surgery. Knee Surg Sports Traumatol Arthrosc 14, 417-424.

Gebhard F, Ellermann A, Hoffmann F, Jaeger JH, Friederich NF (2006): Multicenter-study of operative treatment of intraligamentous tears of the anterior cruciate ligament in children and adolescents: comparison of four different techniques. Knee Surg Sports Traumatol Arthrosc $\underline{14}, 797-803$.

Girgis FG, Marshall JL, Monajem A (1975): The cruciate ligaments of the knee joint. Anatomical, functional and experimental analysis. Clin Orthop Relat Res 1975, 216-231.

Gorin S, Paul DD, Wilkinson EJ (2003): An anterior cruciate ligament and medial collateral ligament tear in a skeletally immature patient: a new technique to augment primary repair of the medial collateral ligament and an allograft reconstruction of the anterior cruciate ligament. Arthroscopy $\underline{19}, 21-26$.

Guzzanti V, Falciglia F, Gigante A, Fabbriciani C (1994): The effect of intra-articular ACL reconstruction on the growth plates of rabbits. J Bone Joint Surg Br 76, 960-963.

Guzzanti V, Falciglia F, Stanitski CL (2003a): Preoperative evaluation and anterior cruciate ligament reconstruction technique for skeletally immature patients in Tanner stages 2 and 3 . Am J Sports Med 31, 941-948.

Guzzanti V, Falciglia F, Stanitski CL (2003b): Physeal-sparing intraarticular anterior cruciate ligament reconstruction in preadolescents. Am J Sports Med 31, 949-953. 
Haus J, Halata Z (1990): Innervation of the anterior cruciate ligament. Int Orthop 14, 293296.

Hefti F, Müller W, Jakob RP, Staubli HU (1993): Evaluation of knee ligament injuries with the IKDC form. Knee Surg Sports Traumatol Arthrosc 1, 226-234.

Henry J, Chotel F, Chouteau J, Fessy MH, Berard J, Moyen B (2009): Rupture of the anterior cruciate ligament in children: early reconstruction with open physes or delayed reconstruction to skeletal maturity? Knee Surg Sports Traumatol Arthrosc 17, 748-755.

Hersekli MA, Akpinar S, Ozalay M, Ozkoc G, Cesur N, Uysal M, Pourbagher A, Tandogan RN (2004): Tunnel enlargement after arthroscopic anterior cruciate ligament reconstruction: comparison of bone-patellar tendon-bone and hamstring autografts. Adv Ther 21, 123-131.

Hertel P, Schweiberer L (1975): [Biomechanics and pathophysiology of the knee ligaments]. Hefte Unfallheilkd, 1-16.

Hoffmann F, Friebel H, Schiller M, Reif G (1998): Versorgung der vorderen Kreuzbandruptur bei offenen Wachstumsfugen. Arthroskopie 11, 28-33.

Inoue M, McGurk-Burleson E, Hollis JM, Woo SL (1987): Treatment of the medial collateral ligament injury. I: The importance of anterior cruciate ligament on the varus-valgus knee laxity. Am J Sports Med 15, 15-21.

Jakob RP, Stäubli HU: Kniegelenk und Kreuzbänder: Anatomie, Biomechanik, Klinik, Rekonstruktion, Komplikationen, Rehabilitation. Springer-Verlag, Berlin 1990

Janarv PM, Nystrom A, Werner S, Hirsch G (1996): Anterior cruciate ligament injuries in skeletally immature patients. J Pediatr Orthop $\underline{16}$, 673-677.

Jardin C, Chantelot C, Migaud H, Gougeon F, Debroucker MJ, Duquennoy A (1999):

[Reliability of the KT-1000 arthrometer in measuring anterior laxity of the knee: comparative analysis with Telos of 48 reconstructions of the anterior cruciate ligament and intra- and interobserver reproducibility]. Rev Chir Orthop Reparatrice Appar Mot 도, 698-707.

Kapandji I: The Physiology of the Joints. (2: lower Limb), 2. Auflage; Churchill Livingstone, Edinburgh 1970

Kellenberger R, von Laer L (1990): Nonosseous lesions of the anterior cruciate ligaments in childhood and adolescence. Prog Pediatr Surg 25, 123-131. 
Kercher J, Xerogeanes J, Tannenbaum A, Al-Hakim R, Black JC, Zhao J (2009): Anterior cruciate ligament reconstruction in the skeletally immature: an anatomical study utilizing 3dimensional magnetic resonance imaging reconstructions. J Pediatr Orthop 29, 124-129.

Knorr J, Accadbled F, Cassard X, Ayel JE, Sales de Gauzy J (2006): [Isolated congenital aplasia of the anterior cruciate ligament treated by reconstruction in a 5-year-old boy]. Rev Chir Orthop Reparatrice Appar Mot 92, 803-808.

Kocabey Y, Tetik O, Isbell WM, Atay OA, Johnson DL (2004): The value of clinical examination versus magnetic resonance imaging in the diagnosis of meniscal tears and anterior cruciate ligament rupture. Arthroscopy $\underline{20}, 696-700$.

Kocher MS, Saxon HS, Hovis WD, Hawkins RJ (2002): Management and complications of anterior cruciate ligament injuries in skeletally immature patients: survey of the Herodicus Society and The ACL Study Group. J Pediatr Orthop 22, 452-457.

Kocher MS, Garg S, Micheli LJ (2005): Physeal sparing reconstruction of the anterior cruciate ligament in skeletally immature prepubescent children and adolescents. J Bone Joint Surg Am ㄱ, 2371-2379.

Kocher MS, Smith JT, Zoric BJ, Lee B, Micheli LJ (2007): Transphyseal anterior cruciate ligament reconstruction in skeletally immature pubescent adolescents. J Bone Joint Surg Am $\underline{89}, 2632-2639$.

Koman JD, Sanders JO (1999): Valgus deformity after reconstruction of the anterior cruciate ligament in a skeletally immature patient. A case report. J Bone Joint Surg Am 1ㅗ, 711-715.

Kunz M (1992): [Injuries of the cruciate ligament in children caused by sports accidents]. Sportverletz Sportschaden $\underline{6}, 2-5$.

Kunz R, Ollenschläger G, Raspe H, Jonitz G, Kolkmann F-W: Lehrbuch Evidenzbasierte Medizin in Klinik und Praxis. 1. Auflage; Deutscher Ärzte-Verlag, Köln 2000

Letts RM, Davidson D, Pressman A (2000): Rekonstruktion des vorderen Kreuzbandes bei Kindern. Oper Orthop Traumatol 12, 146-153.

Liddle AD, Imbuldeniya AM, Hunt DM (2008): Transphyseal reconstruction of the anterior cruciate ligament in prepubescent children. J Bone Joint Surg Br $\underline{90}$, 1317-1322.

Lipscomb AB, Anderson AF (1986): Tears of the anterior cruciate ligament in adolescents. J Bone Joint Surg Am $\underline{68}$, 19-28.

Lo IK, Kirkley A, Fowler PJ, Miniaci A (1997): The outcome of operatively treated anterior cruciate ligament disruptions in the skeletally immature child. Arthroscopy $\underline{13}, 627-634$. 
Lo IK, Bell DM, Fowler PJ (1998): Anterior cruciate ligament injuries in the skeletally immature patient. Instr Course Lect $\underline{47}$, 351-359.

Lukas C, Eberhardt O, Wirth T, Fernandez F (2007): [Results of ACL reconstruction with a periost-patella tendon-periost graft in growth age]. Z Orthop Unfall 145, 706-711.

Lysholm J, Gillquist J (1982): Evaluation of knee ligament surgery results with special emphasis on use of a scoring scale. Am J Sports Med 10, 150-154.

Mah JY, Otsuka NY, McLean J (1996): An arthroscopic technique for the reduction and fixation of tibial-eminence fractures. J Pediatr Orthop $\underline{16}, 119-121$.

Makela EA, Vainionpaa S, Vihtonen K, Mero M, Rokkanen P (1988): The effect of trauma to the lower femoral epiphyseal plate. An experimental study in rabbits. J Bone Joint Surg Br $\underline{70}$, 187-191.

Marshall JL, Warren RF, Wickiewicz TL, Reider B (1979): The anterior cruciate ligament: a technique of repair and reconstruction. Clin Orthop Relat Res 1979, 97-106.

Matava MJ, Siegel MG (1997): Arthroscopic reconstruction of the ACL with semitendinosusgracilis autograft in skeletally immature adolescent patients. Am J Knee Surg 10, 60-69.

McCarroll JR, Rettig AC, Shelbourne KD (1988): Anterior cruciate ligament injuries in the young athlete with open physes. Am J Sports Med 16, 44-47.

McCarroll JR, Shelbourne KD, Porter DA, Rettig AC, Murray S (1994): Patellar tendon graft reconstruction for midsubstance anterior cruciate ligament rupture in junior high school athletes. An algorithm for management. Am J Sports Med 22, 478-484.

McIntosh AL, Dahm DL, Stuart MJ (2006): Anterior cruciate ligament reconstruction in the skeletally immature patient. Arthroscopy 22, 1325-1330.

Menschik A (1974): [Mechanics of the knee-joint]. Z Orthop Ihre Grenzgeb 112, 481-495.

Meyers MH, McKeever FM (1959): Fracture of the intercondylar eminence of the tibia. J Bone Joint Surg Am 41-A, 209-220; discussion 220-202.

Meyers MH, McKeever FM (1970): Fracture of the intercondylar eminence of the tibia. J Bone Joint Surg Am 52, 1677-1684.

Micheli LJ, Foster TE (1993): Acute knee injuries in the immature athlete. Instr Course Lect $\underline{42}, 473-481$. 
Micheli LJ, Coady CM (1997): Anterior Cruciate Ligament Reconstruction in the Pediatric Age Group. Sports Med Arthrosc 5, 106-111.

Micheli LJ, Rask B, Gerberg L (1999): Anterior cruciate ligament reconstruction in patients who are prepubescent. Clin Orthop Relat Res 1999, 40-47.

Millett PJ, Willis AA, Warren RF (2002): Associated injuries in pediatric and adolescent anterior cruciate ligament tears: does a delay in treatment increase the risk of meniscal tear? Arthroscopy $\underline{18}, 955-959$.

Mizuta H, Kubota K, Shiraishi M, Otsuka Y, Nagamoto N, Takagi K (1995): The conservative treatment of complete tears of the anterior cruciate ligament in skeletally immature patients. J Bone Joint Surg Br $\underline{77}$, 890-894.

Mohtadi N, Grant J (2006): Managing anterior cruciate ligament deficiency in the skeletally immature individual: a systematic review of the literature. Clin J Sport Med 16, 457-464.

Moksnes H, Engebretsen L, Risberg MA (2008): Performance-based functional outcome for children 12 years or younger following anterior cruciate ligament injury: a two to nine-year follow-up study. Knee Surg Sports Traumatol Arthrosc 16, 214-223.

Müller W (1975): [Rotational stability in the knee joint]. Hefte Unfallheilkd 1975, 51-68.

Müller W (1977): [Lesions of the cruciate ligaments]. Zentralbl Chir 102, 974-981.

Nakhostine M, Bollen SR, Cross MJ (1995): Reconstruction of mid-substance anterior cruciate rupture in adolescents with open physes. J Pediatr Orthop $\underline{15}$, 286-287.

Noyes FR, Butler DL, Grood ES, Zernicke RF, Hefzy MS (1984): Biomechanical analysis of human ligament grafts used in knee-ligament repairs and reconstructions. J Bone Joint Surg Am $\underline{66}, 344-352$.

Odensten M, Gillquist J (1985): Functional anatomy of the anterior cruciate ligament and a rationale for reconstruction. J Bone Joint Surg Am $\underline{67}$, 257-262.

Oxford Centre (2009), http://www.cebm.net/index.aspx?o=1025, Abrufdatum: 26.10.2009

Papageorgiou CD, Gil JE, Kanamori A, Fenwick JA, Woo SL, Fu FH (2001): The biomechanical interdependence between the anterior cruciate ligament replacement graft and the medial meniscus. Am J Sports Med 29, 226-231.

Parker AW, Drez D, Jr. Cooper JL (1994): Anterior cruciate ligament injuries in patients with open physes. Am J Sports Med 22, 44-47. 
Petersen W, Zantop T (2007): Anatomy of the anterior cruciate ligament with regard to its two bundles. Clin Orthop Relat Res $\underline{454}$, 35-47.

Petersen W, Rosenbaum D, Raschke M (2005): Rupturen des vorderen Kreuzbandes bei weiblichen Athleten. Teil 1: Epidemiologie, Verletzungsmechanismen und Ursachen. Dtsch Z Sportmed 56, 150-156.

Piziali RL, Seering WP, Nagel DA, Schurman DJ (1980): The function of the primary ligaments of the knee in anterior-posterior and medial-lateral motions. J Biomech $\underline{13}$, 777 784.

Pous JG, Baldet P, Dimeglio A, Bonnel F (1979): [Injuries of the epiphyseal cartilage. Fundamental concepts]. Rev Chir Orthop Reparatrice Appar Mot 65, 259-265.

Pressman AE, Letts RM, Jarvis JG (1997): Anterior cruciate ligament tears in children: an analysis of operative versus nonoperative treatment. J Pediatr Orthop $\underline{17}$, 505-511.

Reilly CW, Choit RL (2007): Proximal migration of femoral staples after ACL reconstruction in a skeletally immature patient. Orthopedics $\underline{30}, 575-576$.

Rinaldi E, Mazzarella F (1980): Isolated fracture-avulsions of the tibial insertions of the cruciate ligaments of the knee. Ital J Orthop Traumatol $\underline{6}, 77-83$.

Risberg MA, Holm I, Steen H, Beynnon BD (1999): Sensitivity to changes over time for the IKDC form, the Lysholm score, and the Cincinnati knee score. A prospective study of 120 ACL reconstructed patients with a 2-year follow-up. Knee Surg Sports Traumatol Arthrosc 7 , 152-159.

Robert H, Bonnard C (1999): The possibilities of using the patellar tendon in the treatment of anterior cruciate ligament tears in children. Arthroscopy 15, 73-76.

Rush WA, Steiner HA (1946): A study of lower extremity length inequality. Am J Roentgenol Radium Ther $\underline{56}$, 616-623.

Sackett DL, Straus S, Richardson WS, Rosenberg W, Haynes BR: Evidence-basend Medicine. Churchill Livingstone, Edinburgh 2000

Sankar WN, Wells L, Sennett BJ, Wiesel BB, Ganley TJ (2006): Combined anterior cruciate ligament and medial collateral ligament injuries in adolescents. J Pediatr Orthop 26 6 , 733-736.

Scavenius M, Bak K, Hansen S, Norring K, Jensen KH, Jorgensen U (1999): Isolated total ruptures of the anterior cruciate ligament - a clinical study with long-term follow-up of 7 years. Scand J Med Sci Sports 9, 114-119. 
Schachter AK, Rokito AS (2007): ACL injuries in the skeletally immature patient. Orthopedics $\underline{30}$, 365-370; quiz 371-372.

Schaefer RA, Eilert RE, Gillogly SD (1993): Disruption of the anterior cruciate ligament in a 4-year-old child. Orthop Rev 22, 725-727.

Schmidt-Wiethoff R, Dargel J (2007): Aktuelle Konzepte zur Diagnose und Therapie der vorderen Kreuzbandruptur. Dtsch Z Sportmed 58, 384-391.

Schultz A, Skorpik G, Gaudernak T (1996): Ergebnisse der operierten vorderen Kreuzbandruptur bei Kindern. Acta Chir Austriaca 28, 25-26.

Schutte MJ, Dabezies EJ, Zimny ML, Happel LT (1987): Neural anatomy of the human anterior cruciate ligament. J Bone Joint Surg Am $\underline{69}$, 243-247.

Seil R: Die Ersatzplastik des vorderen Kreuzbandes bei offenen Wachstumsfugen - eine experimentelle Untersuchung. Med. Habil. Homburg/Saar 2002.

Seil R, Robert H (2005): VKB-Plastik bei offenen Wachstumsfugen. Arthroskopie 18, 48-52.

Seil R, Pape D, Kohn D (2008): The risk of growth changes during transphyseal drilling in sheep with open physes. Arthroscopy 24, 824-833.

Seon JK, Song EK, Yoon TR, Park SJ (2005): Transphyseal reconstruction of the anterior cruciate ligament using hamstring autograft in skeletally immature adolescents. J Korean Med Sci 20, 1034-1038.

Sharma L, Song J, Dunlop D, Felson D, Lewis CE, Segal N, Torner J, Cooke TD, Hietpas J, Lynch J, et al. (2010): Varus and valgus alignment and incident and progressive knee osteoarthritis. Ann Rheum Dis $\underline{69}, 1940-1945$.

Shea KG, Apel PJ, Pfeiffer RP (2003): Anterior cruciate ligament injury in paediatric and adolescent patients: a review of basic science and clinical research. Sports Med 33, 455-471.

Shea KG, Apel PJ, Pfeiffer RP, Traughber PD (2007): The anatomy of the proximal tibia in pediatric and adolescent patients: implications for ACL reconstruction and prevention of physeal arrest. Knee Surg Sports Traumatol Arthrosc 15, 320-327.

Shelbourne KD, Gray T, Wiley BV (2004): Results of transphyseal anterior cruciate ligament reconstruction using patellar tendon autograft in tanner stage 3 or 4 adolescents with clearly open growth plates. Am J Sports Med 32, 1218-1222.

Siliski JM: Traumatic disorders of the knee. Springer-Verlag, New York 1994 
Skak SV, Jensen TT, Poulsen TD, Sturup J (1987): Epidemiology of knee injuries in children. Acta Orthop Scand $\underline{58}, 78-81$.

Sobau C, Ellermann A (2004): [Anterior cruciate ligament reconstruction with hamstring tendons in the young]. Unfallchirurg 107, 676-679.

Sobotta J, Becher H: Atlas der Anatomie des Menschen. (1. Band: Regionen, Knochen, Bänder, Gelenke und Muskeln), 17. Auflage; Urban \& Schwarzenberg, München 1972

Stadelmaier DM, Arnoczky SP, Dodds J, Ross H (1995): The effect of drilling and soft tissue grafting across open growth plates. A histologic study. Am J Sports Med 23, 431-435.

Steadman JR, Cameron-Donaldson ML, Briggs KK, Rodkey WG (2006): A minimally invasive technique ("healing response") to treat proximal ACL injuries in skeletally immature athletes. J Knee Surg 19, 8-13.

Strecker W (2006): Planning analysis of knee-adjacent deformities. I. Frontal plane deformities. Oper Orthop Traumatol 18, 259-272.

Strecker W, Keppler P (2002): [Analysis and correction of leg deformities. 1: Analysis]. Unfallchirurg 105, 811-829.

Strobel MJ, Stedtfeld HW, Eichhorn J: Diagnostik des Kniegelenkes. 3. Auflage; Springer Verlag, Berlin 1995.

Tallay A, Lim MH, Morris HG (2008): Living related donor allograft for revision anterior cruciate ligament reconstruction in a child: a case report. Knee $\underline{15}, 407-410$.

Thompson M, Flynn J, Wells L, Ganley TJ (2006): Single incision arthroscopic ACL reconstruction in skeletally immature patients with direct visualization of the femoral and tibial physes. Orthopedics $\underline{29}$, 488-492.

Tyler TF, McHugh MP, Gleim GW, Nicholas SJ (1999): Association of KT-1000 measurements with clinical tests of knee stability 1 year following anterior cruciate ligament reconstruction. J Orthop Sports Phys Ther 29, 540-545.

Vaquero J, Vidal C, Cubillo A (2005): Intra-articular traumatic disorders of the knee in children and adolescents. Clin Orthop Relat Res 2005, 97-106.

von Recum J, Schmickal T, Hochstein P, Wentzensen A (2002): Verletzungen des kindlichen Kniegelenks. Trauma Berufskrankh $\underline{4}$, 20-29.

Wagner M, Schabus R: Funktionelle Anatomie des Kniegelenks. Springer-Verlag, Heidelberg 1982 
Wahler G, Neubauer T, Knotzer M, Wagner M (1998): Operative Therapie der Ruptur des vorderen Kreuzbandes (LCA) bei Kindern und Jugendlichen. Acta Chir Austriaca 30 (Suppl. $\underline{143)}, 112-114$.

Westhoff B, Jager M, Krauspe R (2007): [Axes of the legs in childhood. What is pathologic?]. Orthopade 36, 485-498; quiz 499-500.

Wirth C, Zichner L: Orthopädie und Orthopädische Chirurgie. Georg Thieme Verlag, Stuttgart 2005

Woods GW, O'Connor DP (2004): Delayed anterior cruciate ligament reconstruction in adolescents with open physes. Am J Sports Med 32, 201-210.

Wroble RR, Van Ginkel LA, Grood ES, Noyes FR, Shaffer BL (1990): Repeatability of the KT-1000 arthrometer in a normal population. Am J Sports Med 18, 396-399.

Zaricznyj B (1977): Avulsion fracture of the tibial eminence: treatment by open reduction and pinning. J Bone Joint Surg Am 모, 1111-1114. 


\section{Abbildungsverzeichnis}

Abbildung 1: Rechtes Kniegelenk eines Erwachsenen, in gebeugter Stellung nach Entfernung der Gelenkkapsel und der Seitenbänder. Aus: Sobotta und Becher (1972), S. 127. Mit freundlicher Genehmigung des Elsevier-Verlags.

Abbildung 2: Kondylen der Tibia mit den Menisken und den Ursprüngen der beiden Kreuzbänder. Aus: Sobotta und Becher (1972), S. 127. Mit freundlicher Genehmigung des Elsevier-Verlags.

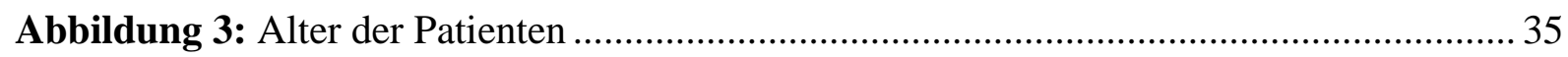

Abbildung 4: Femorale Fixierung des Transplantats.......................................................... 37

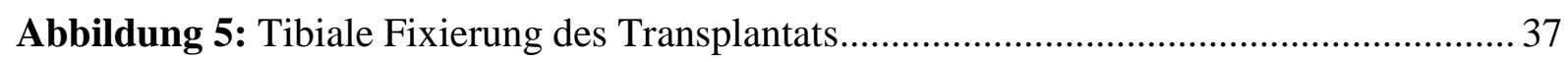

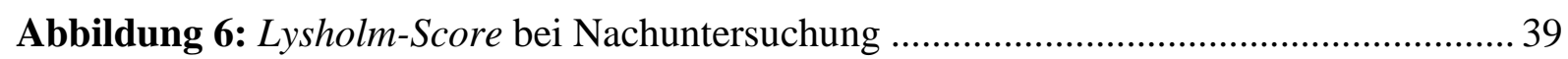

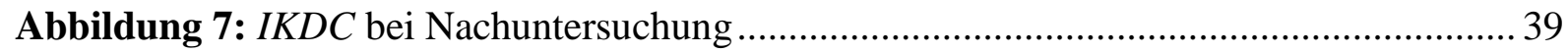

Abbildung 8: Gemessene Bandlaxizität (KT-1000) bei Nachuntersuchung relativ zur

Gegenseite 40

Abbildung 9: Fehlwachstum im Vergleich zur Gegenseite zum Zeitpunkt der Nachuntersuchung....

Abbildung 10: Entwicklung einer postoperativen Beinlängendifferenz oder

Achsenabweichung in Abhängigkeit von der Operationsmethode

Abbildung 11: Ablauf der Studienselektion 44

Abbildung 12: Nachuntersuchungsbogen der Multicenterstudie, Seite 1 84

Abbildung 13: Nachuntersuchungsbogen der Multicenterstudie, Seite 2 85

Abbildung 14: Nachuntersuchungsbogen der Multicenterstudie, Seite 3 .86

Abbildung 15: Nachuntersuchungsbogen der Multicenterstudie, Seite 4. 


\section{Tabellenverzeichnis}

Tabelle 1: Levels of Evidence für Therapie- und Präventionsstudien (modifiziert nach Oxford Centre 2009, http://www.cebm.net/index.aspx?o=1025). 32

Tabelle 2: Operationsergebnisse nach unterschiedlichen Methoden (1) ................................ 42

Tabelle 3: Operationsergebnisse nach unterschiedlichen Methoden (2) ............................... 42

Tabelle 4: Kreuzbandplastik: Therapieergebnis im Wachstumsalter (bis maximal zwölf Jahre bei OP)

Tabelle 5: Kreuzbandplastik im Wachstumsalter (bis maximal zwölf Jahre bei OP):

Wachstumsfugenschonende Operationstechniken versus transphysäre Techniken 45

Tabelle 6: Kreuzbandplastik bei Patienten im Wachstumsalter (altersunabhängig): wachstumsfugenschonende versus wachstumsfugenkreuzende Techniken

Tabelle 7: Kreuzbandplastik im Wachstumsalter (bis maximal zwölf Jahre bei OP):

Ergebnisse in Abhängigkeit vom verwendeten Transplantat

Tabelle 8: Kreuzbandplastik im Wachstumsalter (altersunabhängig): Ergebnisse in

Abhängigkeit vom verwendeten Transplantat.

Tabelle 9: Kreuzbandplastik im Wachstumsalter (bis maximal zwölf Jahre bei OP): Femoral und tibial gelenkferne versus beidseitig gelenknahe Transplantatfixierung 50

Tabelle 10: Kreuzbandplastik im Wachstumsalter (altersunabhängig): Femoral und tibial gelenkferne versus beidseits gelenknahe Transplantatfixierung....

Tabelle 11: Kreuzbandplastik im Wachstumsalter (bis maximal zwölf Jahre bei OP): Anlage des femoralen Bohrkanals über das anteromediale Portal gegenüber einer transtibialen Anlage des Bohrkanals

Tabelle 12: Kreuzbandplastik im Wachstumsalter (altersunabhängig): Anlage des femoralen Bohrkanals über das anteromediale Portal gegenüber einer transtibialen Anlage des Bohrkanals

Tabelle 13: Analyse der Beinlängendifferenzen oder Achsenabweichungen nach Kreuzbandplastik im Wachstumsalter: Vergleich der Altersgruppen

Tabelle 14: Kreuzbandnaht: Therapieergebnisse bei Patienten bis zu einem Alter von zwölf Jahren gegenüber Patienten ab 13 Jahren zum Zeitpunkt der OP.

Tabelle 15: Kreuzbandnaht versus Kreuzbandplastik bei Patienten im Wachstumsalter (altersunabhängig) 55

Tabelle 16: Daten der Multicenterstudie.... 88

Tabelle 17: Daten der Patienten bis zu zwölf Jahren aus der systematischen Literaturanalyse

Tabelle 18: Beinlängendifferenzen und Achsenabweichungen aus der systematischen Literaturanalyse 96 


\section{Danksagung}

Mein besonderer Dank gilt Herrn Prof. Dr. med. Karl-Heinz Frosch für die Überlassung dieses interessanten Themas und die Möglichkeit der Promotion in der Abteilung Unfallchirurgie, Plastische und Wiederherstellungschirurgie der Medizinischen Fakultät der Georg-August-Universität Göttingen. Vor allem danke ich ihm für die außergewöhnlich gute Zusammenarbeit, engagierte Unterstützung, die zahlreichen Gespräche und die Betreuung bei der Durchführung und Auswertung der Studie. Außerdem möchte ich ihm herzlich für das Korrekturlesen der Arbeit und für seine wertvollen Anregungen danken. Bei Herrn Dr. Klaus Jung aus der Abteilung Medizinische Statistik der Universitätsmedizin Göttingen möchte ich mich für die freundliche Beratung und Unterstützung bedanken. 